\title{
Development of a Green \& Sustainable Manufacturing Process for Gefapixant Citrate (MK-7264) Part 3: Development of a One-Pot Formylation-Cyclization Sequence to the Diaminopyrimidine Core
}

Kallol Basu, ${ }^{1, *}$ Dan Lehnherr, ${ }^{1, *}$ Gary E. Martin, ${ }^{2}$ Richard A. Desmond, ${ }^{1}$ Yu-hong Lam, ${ }^{3}$ Feng Peng, ${ }^{1}$ John Y. L. Chung, ${ }^{1}$ Rebecca A. Arvary, ${ }^{1}$ Michael A. Zompa, ${ }^{1}$ Si-Wei Zhang, ${ }^{1}$ Jinchu Liu, ${ }^{1}$ Zachary E. X. Dance, ${ }^{4}$ Patrick Larpent, ${ }^{1}$ Ryan D. Cohen, ${ }^{2}$ Francisco J. Guzman, ${ }^{1}$ Nicholas J. Rogus, ${ }^{1}$ Michael J. Di Maso, ${ }^{1}$ Hong Ren, ${ }^{1}$ Kevin M. Maloney ${ }^{1}$

${ }^{1}$ Department of Process Research and Development, Merck \& Co., Inc., Rahway, New Jersey, 07065 , United States

${ }^{2}$ Structure Elucidation Group, Analytical Research and Development, Merck \& Co., Inc., Rahway, New Jersey, 07065, United States

${ }^{3}$ Computational and Structural Chemistry, Merck \& Co., Inc., Rahway, New Jersey, 07065, United States

${ }^{4}$ Data Rich Measurements, Analytical Research and Development, Merck \& Co., Inc., Rahway, New Jersey, 07065, United States

kallol.basu@merck.com

dan.lehnherr@merck.com

\section{Table of Contents}

1. NMR Spectra. $\mathrm{S} 2$

2. DFT Computational Study

2.1. Computational Method for Modeling the Reactions in Scheme 2 ...

2.2. Cartesian Coordinates of DFT Optimized Structures in Scheme 2

2.3. DFT Calculations and Analysis of NOESY Spectrum for Assignment of the Stereochemistry of Compound $\mathbf{8 a}$

3. References 


\section{NMR Spectra}

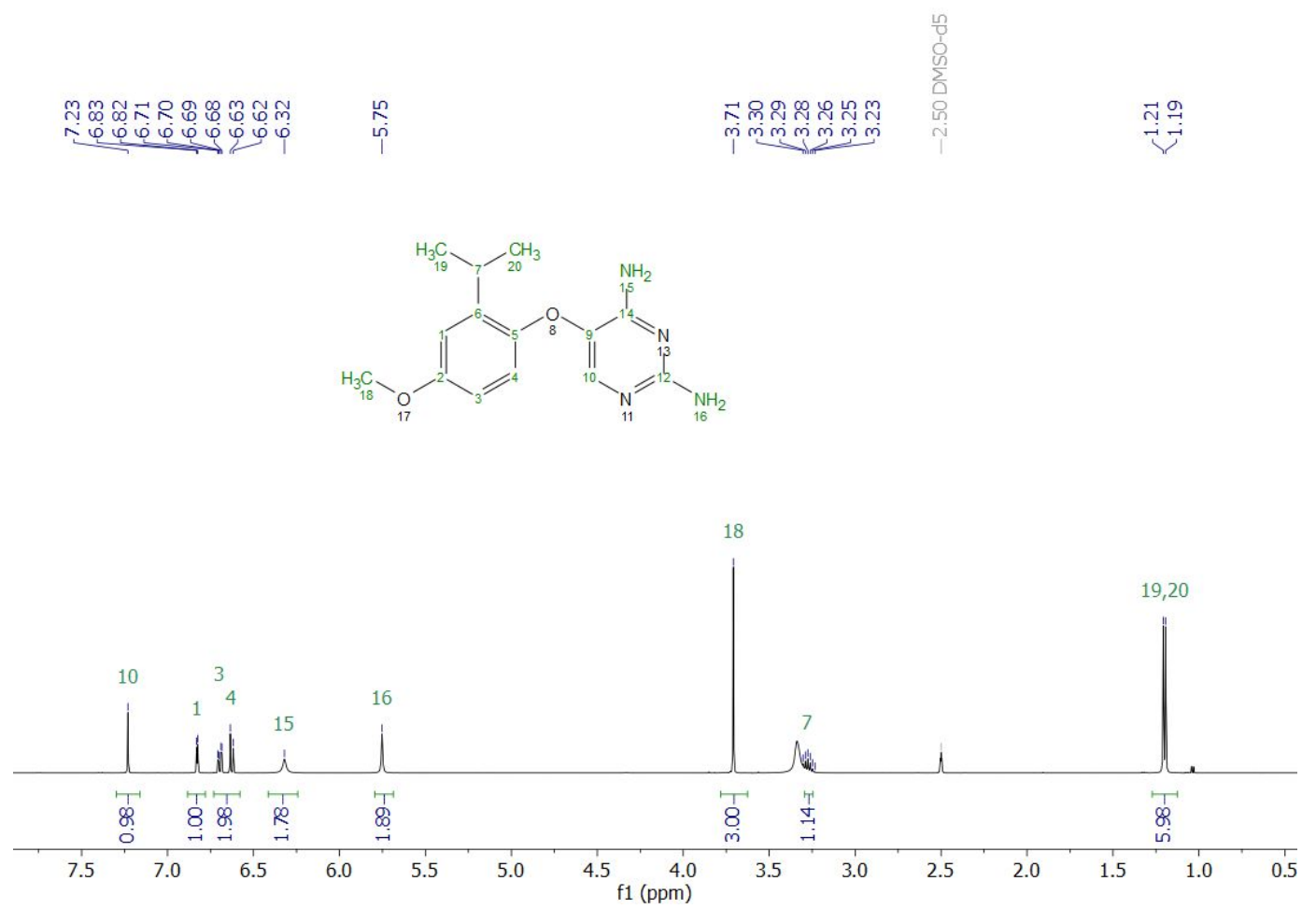

Figure S1. ${ }^{1} \mathrm{H}$ NMR $(500 \mathrm{MHz})$ spectrum of 1 in $d_{6}$-DMSO.
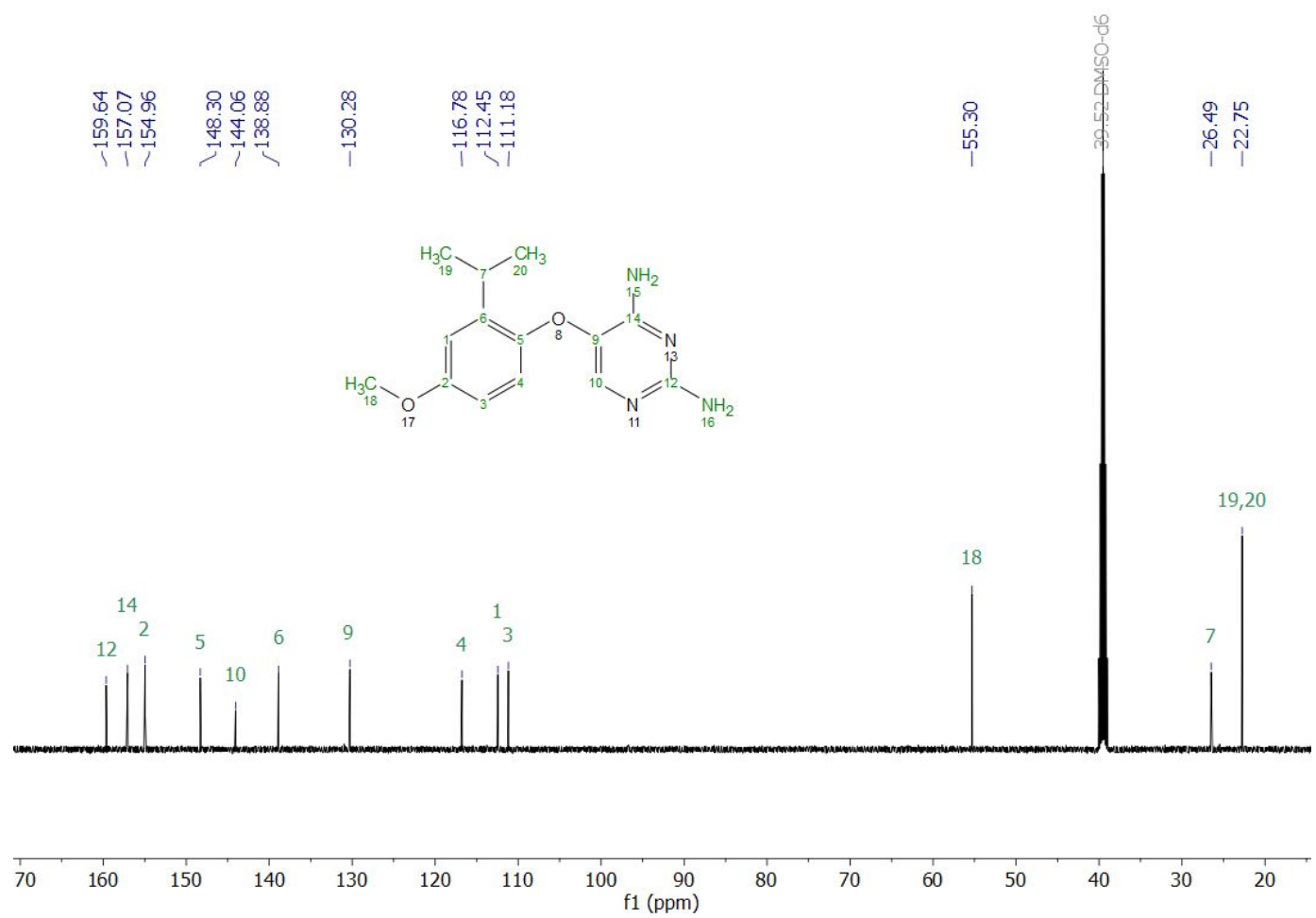

Figure S2. ${ }^{13} \mathrm{C}$ NMR $(126 \mathrm{MHz})$ spectrum of 1 in $d_{6}$-DMSO. 


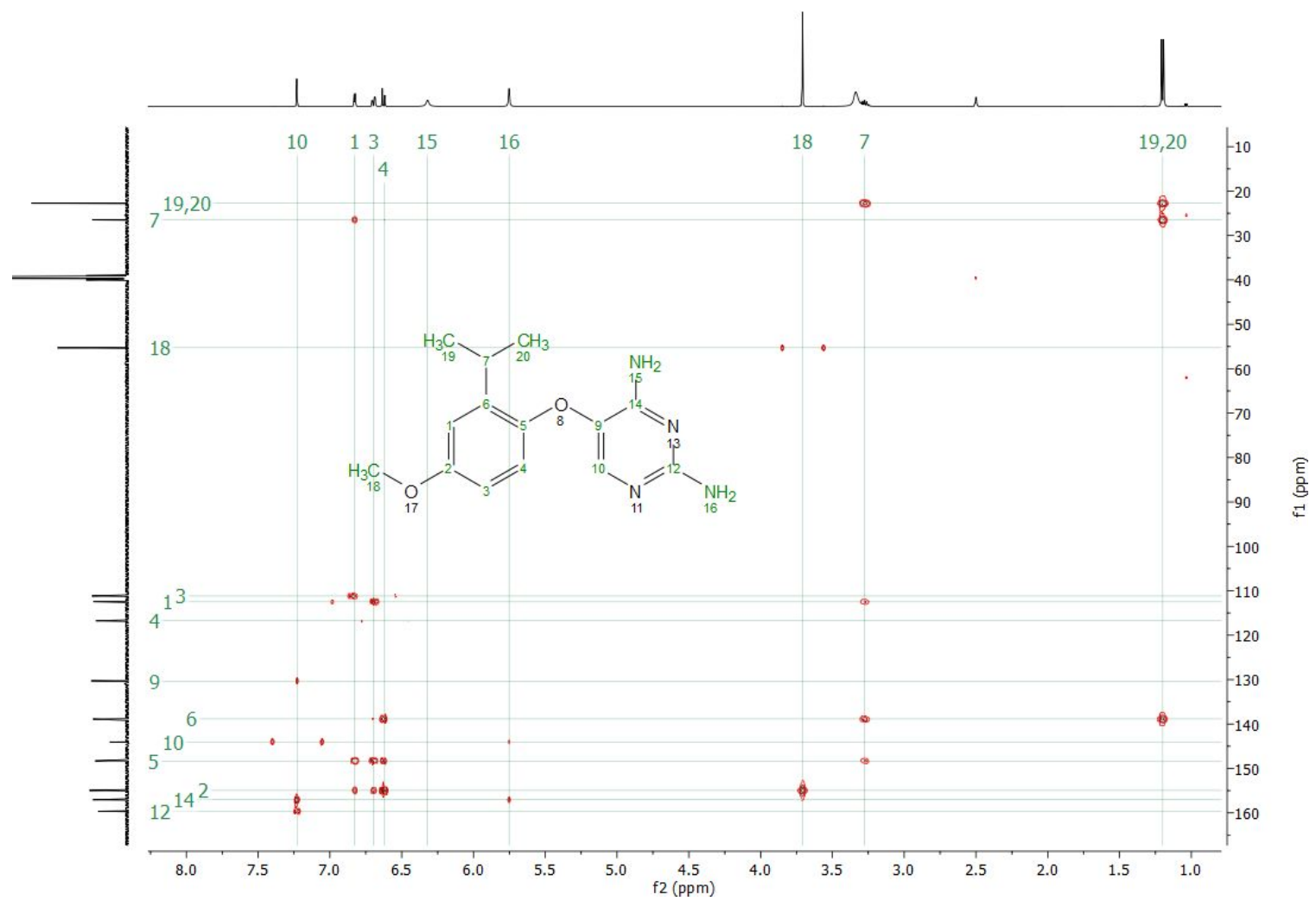

Figure S3. ${ }^{1} \mathrm{H}-{ }^{13} \mathrm{C}$ HMBC NMR $(500 / 126 \mathrm{MHz})$ spectrum of 1 in $d_{6}$-DMSO.

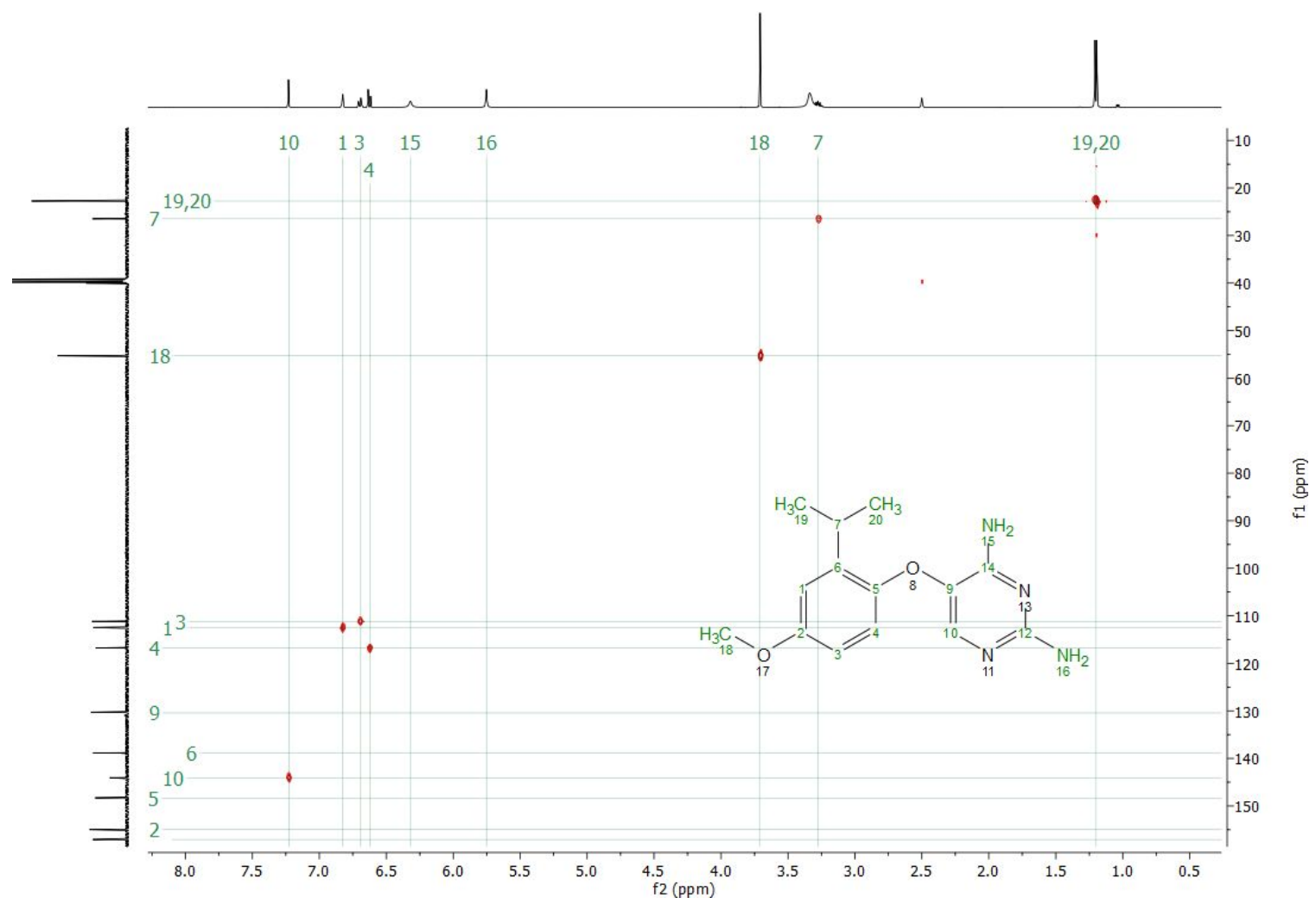

Figure S4. ${ }^{1} \mathrm{H}-{ }^{13} \mathrm{C}$ HSQC NMR (500/126 MHz) spectrum of 1 in $d_{6}$-DMSO. 


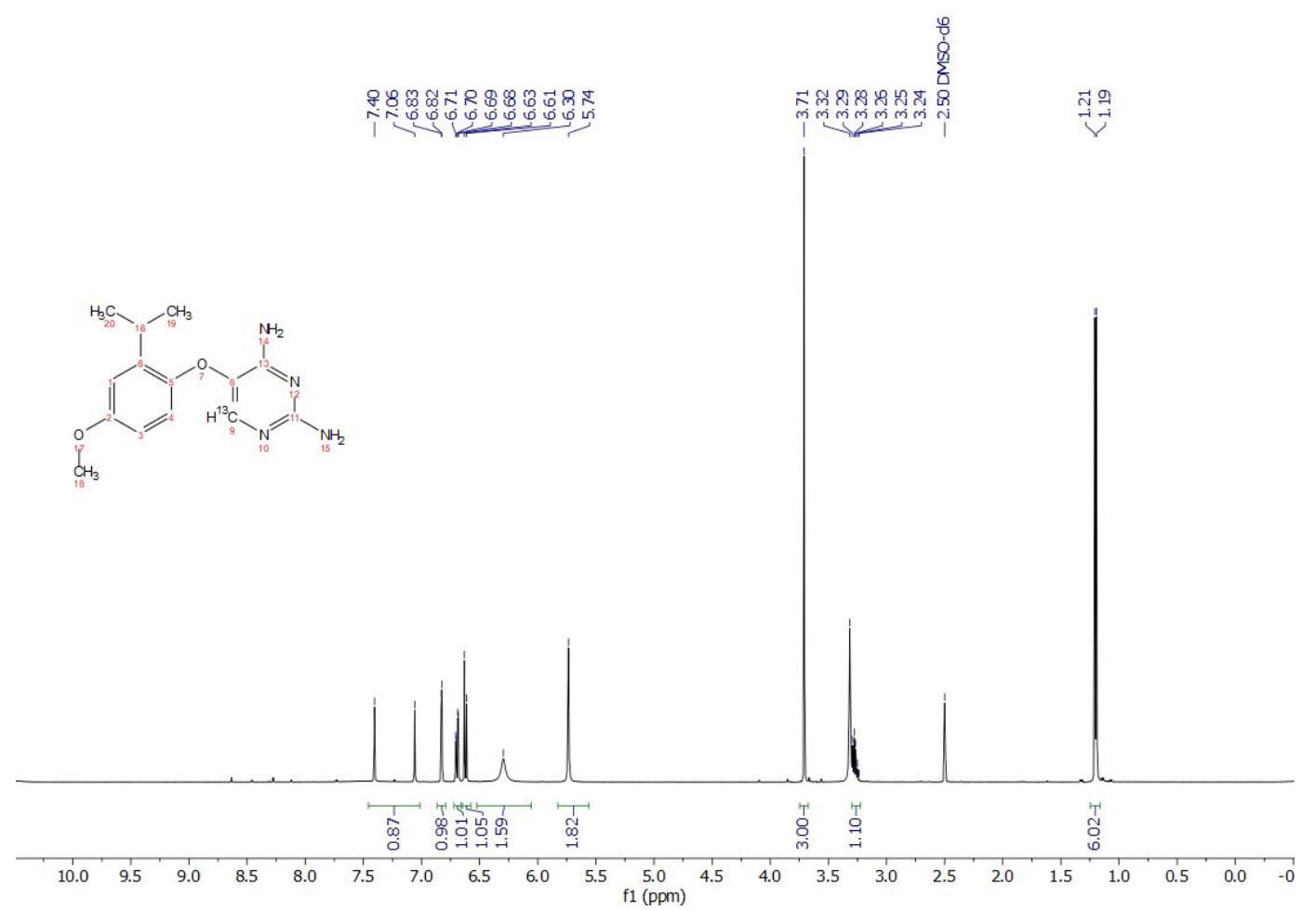

Figure S5. ${ }^{1} \mathrm{H}$ NMR $(500 \mathrm{MHz})$ spectrum of $6-{ }^{13} \mathrm{C}-1$ in $d_{6}$-DMSO.

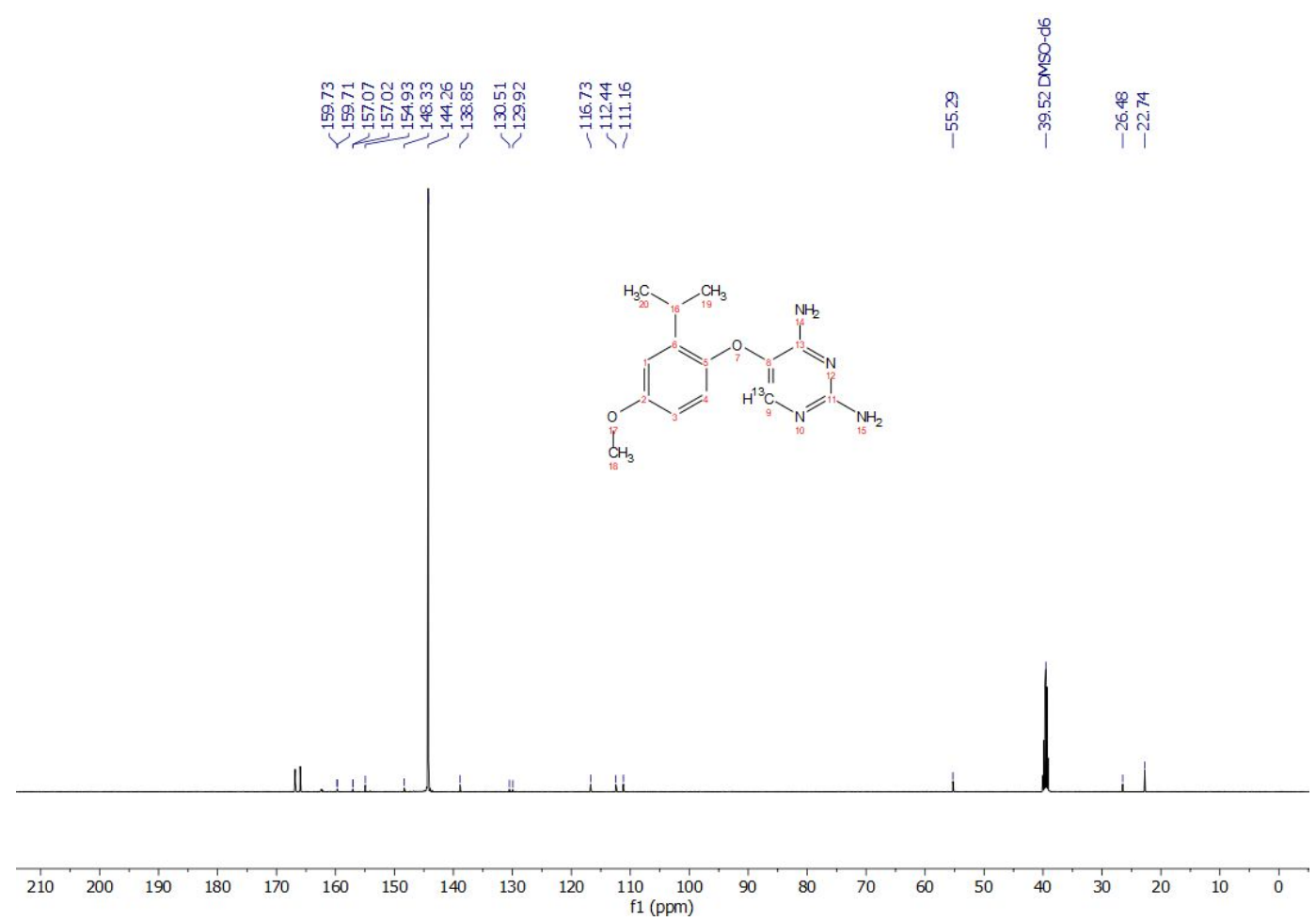

Figure S6. ${ }^{13} \mathrm{C}$ NMR $(126 \mathrm{MHz})$ spectrum of $6-{ }^{13} \mathrm{C}-1$ in $d_{6}$-DMSO. 


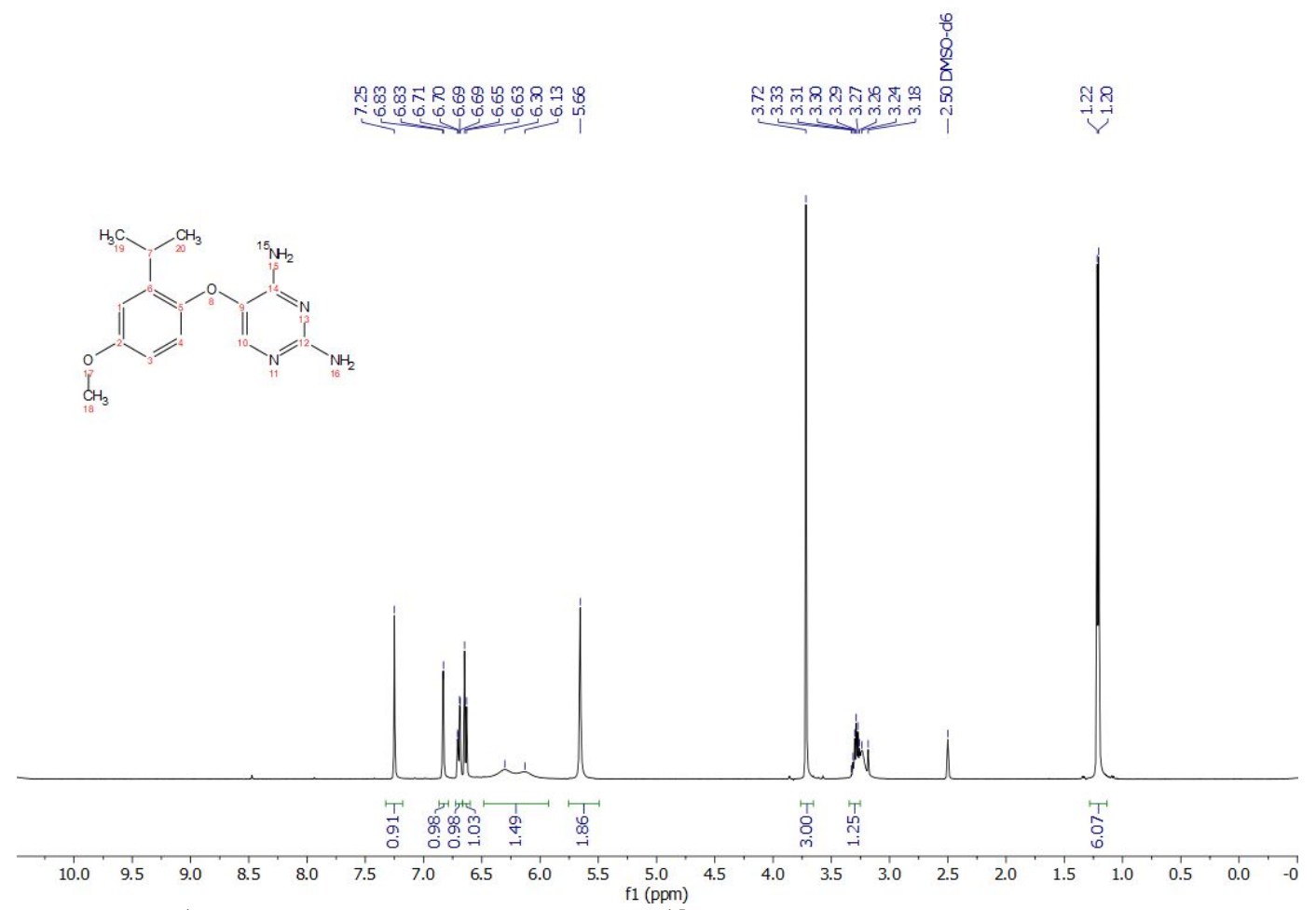

Figure S7. ${ }^{1} \mathrm{H}$ NMR (500 MHz) spectrum of ${ }^{15} \mathrm{~N}-1$ in $d_{6}$-DMSO.

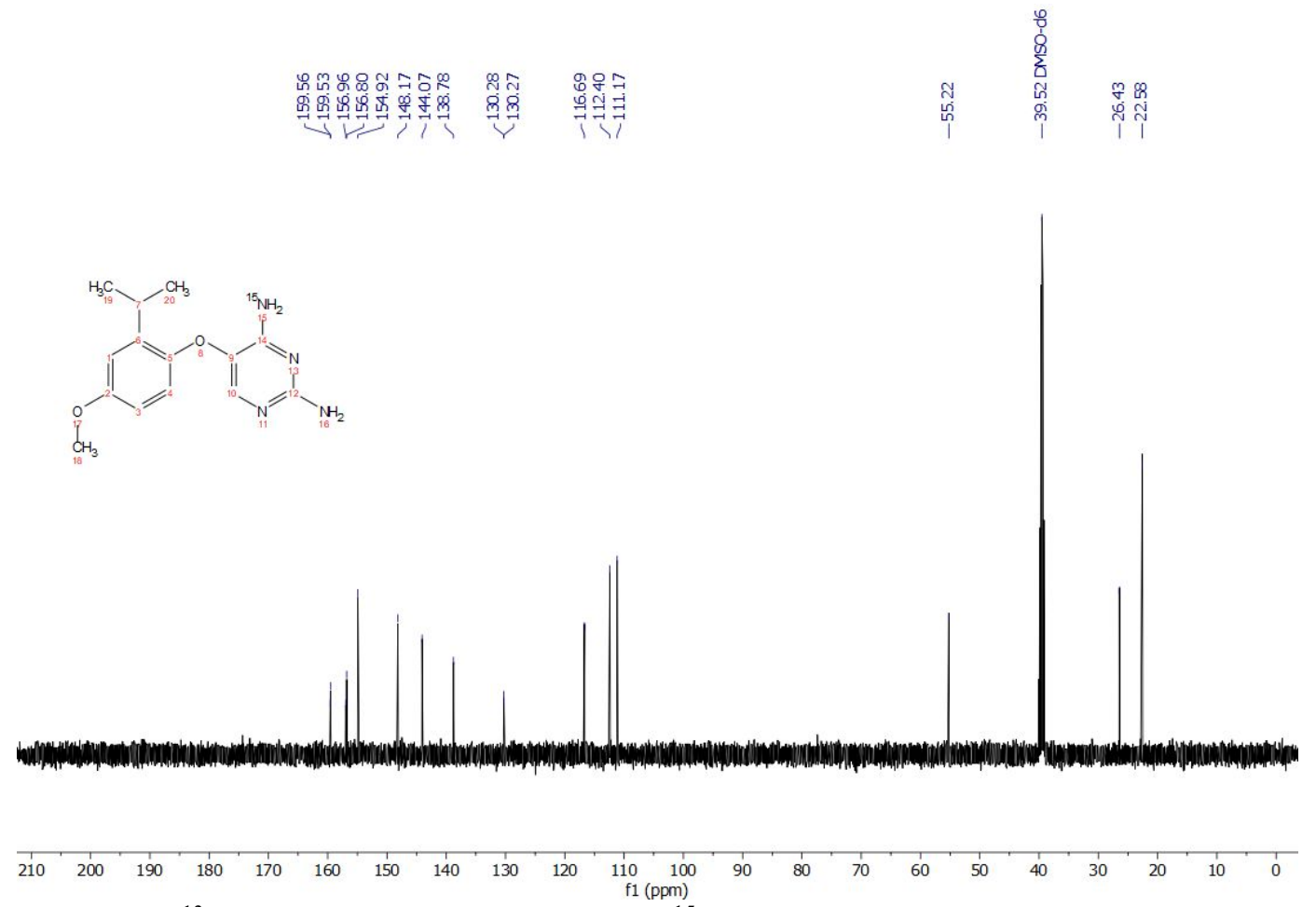

Figure S8. ${ }^{13} \mathrm{C}$ NMR $(126 \mathrm{MHz})$ spectrum of ${ }^{15} \mathrm{~N}-1$ in $d_{6}$-DMSO. 


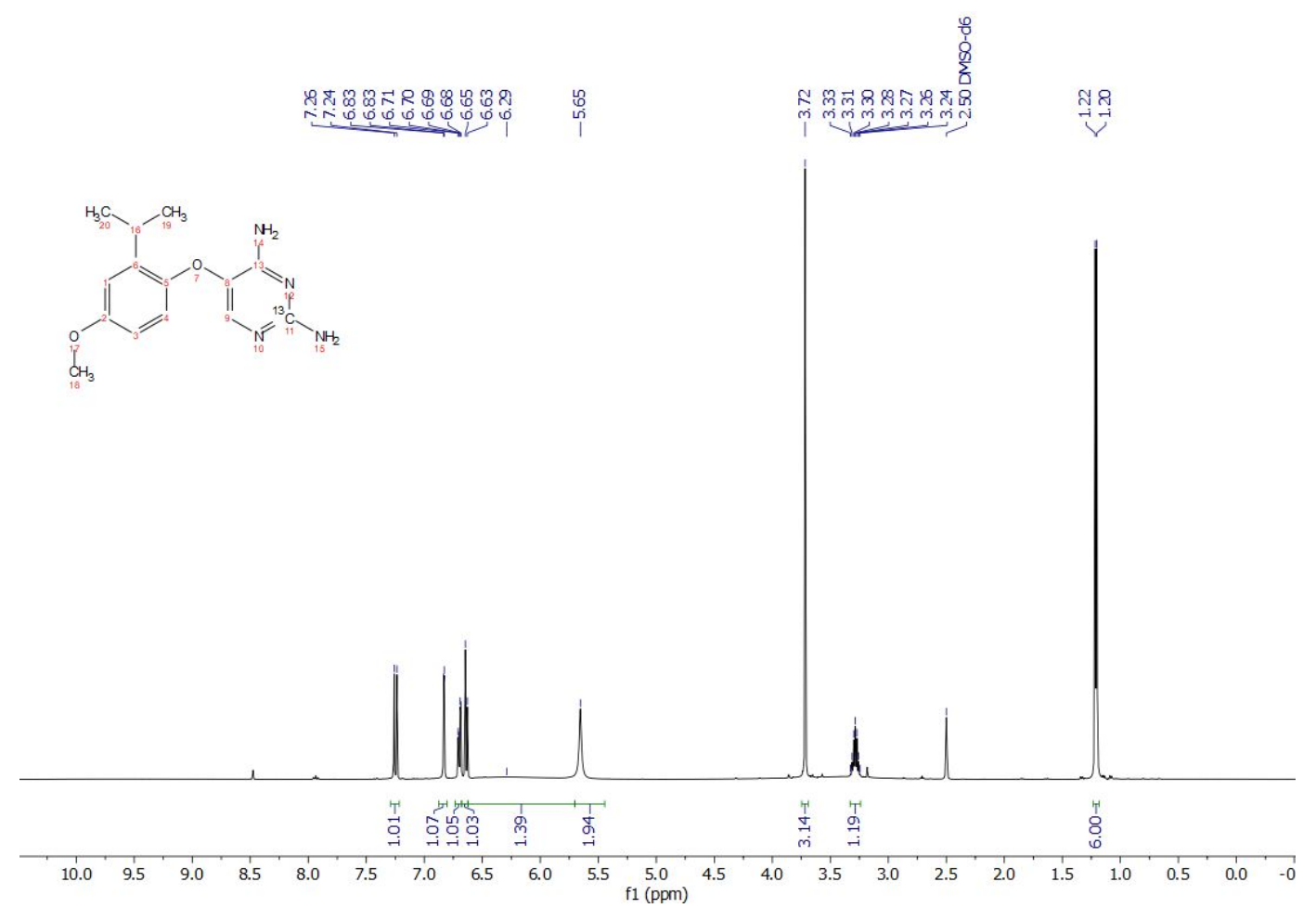

Figure S9. ${ }^{1} \mathrm{H}$ NMR $(500 \mathrm{MHz})$ spectrum of $2-{ }^{13} \mathrm{C}-1$ in $d_{6}$-DMSO.

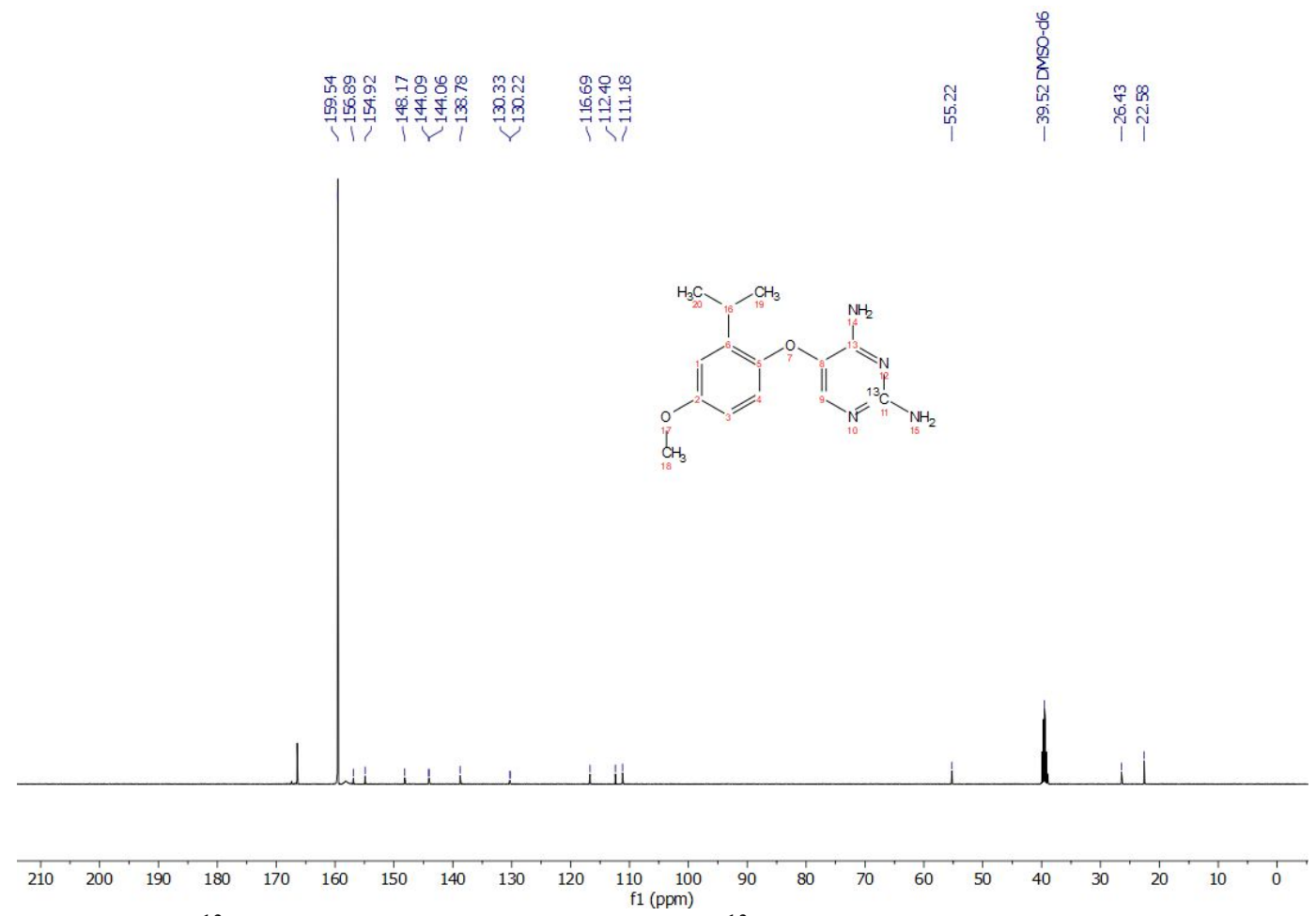

Figure S10. ${ }^{13} \mathrm{C}$ NMR $(126 \mathrm{MHz})$ spectrum of $2-{ }^{13} \mathrm{C}-1$ in $d_{6}$-DMSO. 


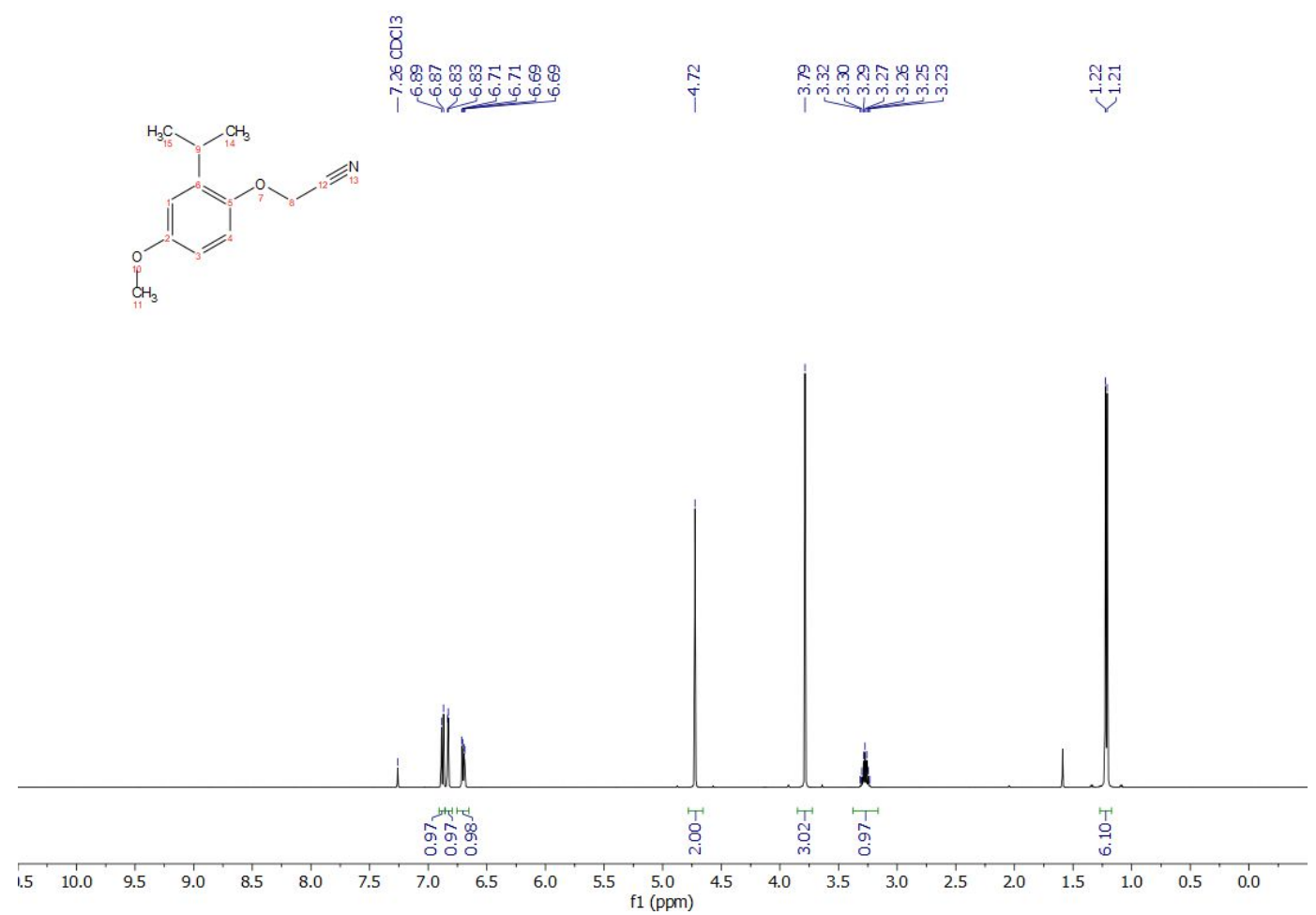

Figure S11. ${ }^{1} \mathrm{H}$ NMR $(500 \mathrm{MHz})$ spectrum of 2 in $\mathrm{CDCl}_{3}$.

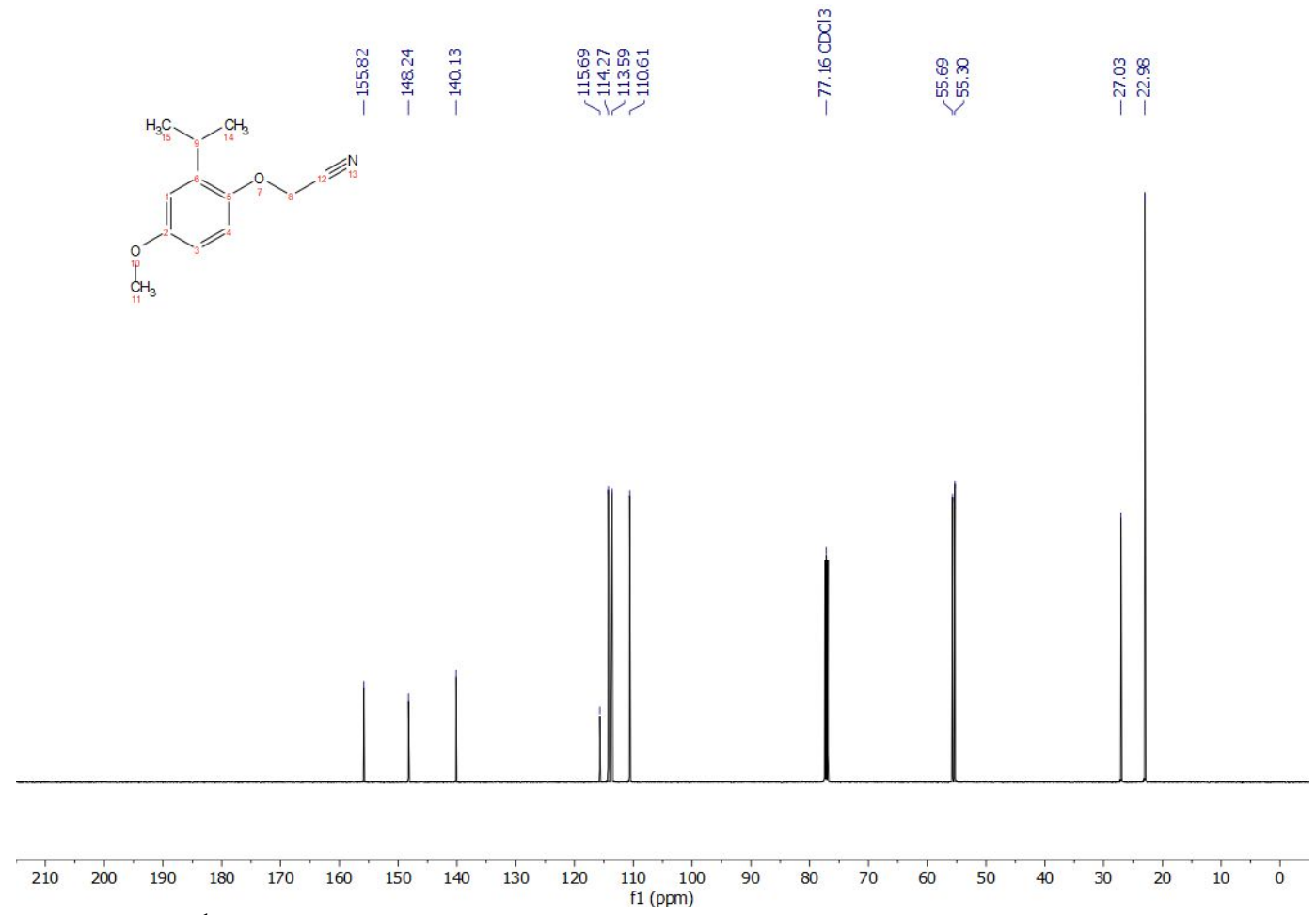

Figure S12. ${ }^{1} \mathrm{H}$ NMR (126 MHz) spectrum of 2 in $\mathrm{CDCl}_{3}$. 

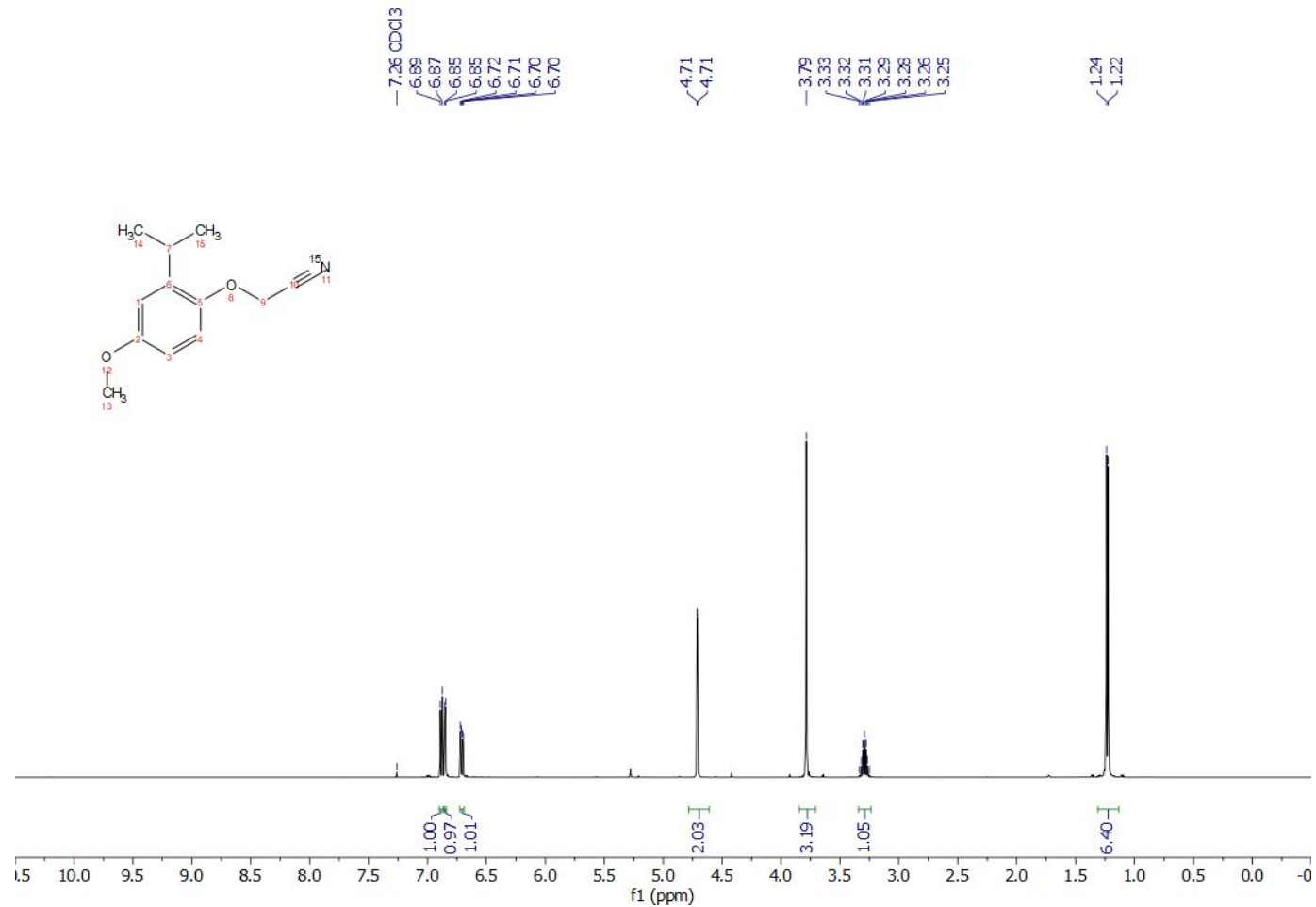

Figure S13. ${ }^{1} \mathrm{H}$ NMR $(500 \mathrm{MHz})$ spectrum of ${ }^{15} \mathrm{~N}-2$ in $\mathrm{CDCl}_{3}$.

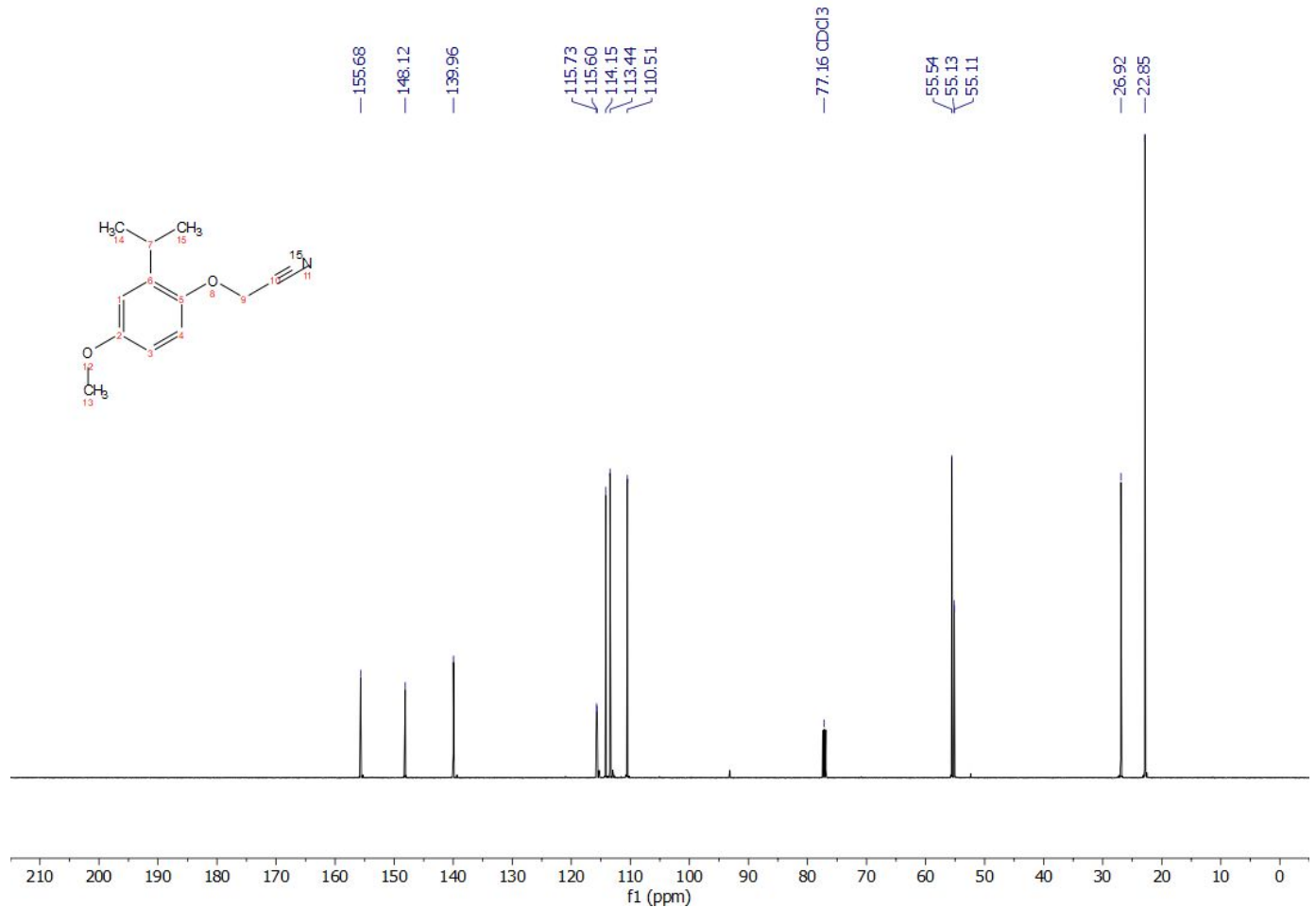

Figure S14. ${ }^{13} \mathrm{C}$ NMR $(500 \mathrm{MHz})$ spectrum of ${ }^{15} \mathrm{~N}-2$ in $\mathrm{CDCl}_{3}$. 


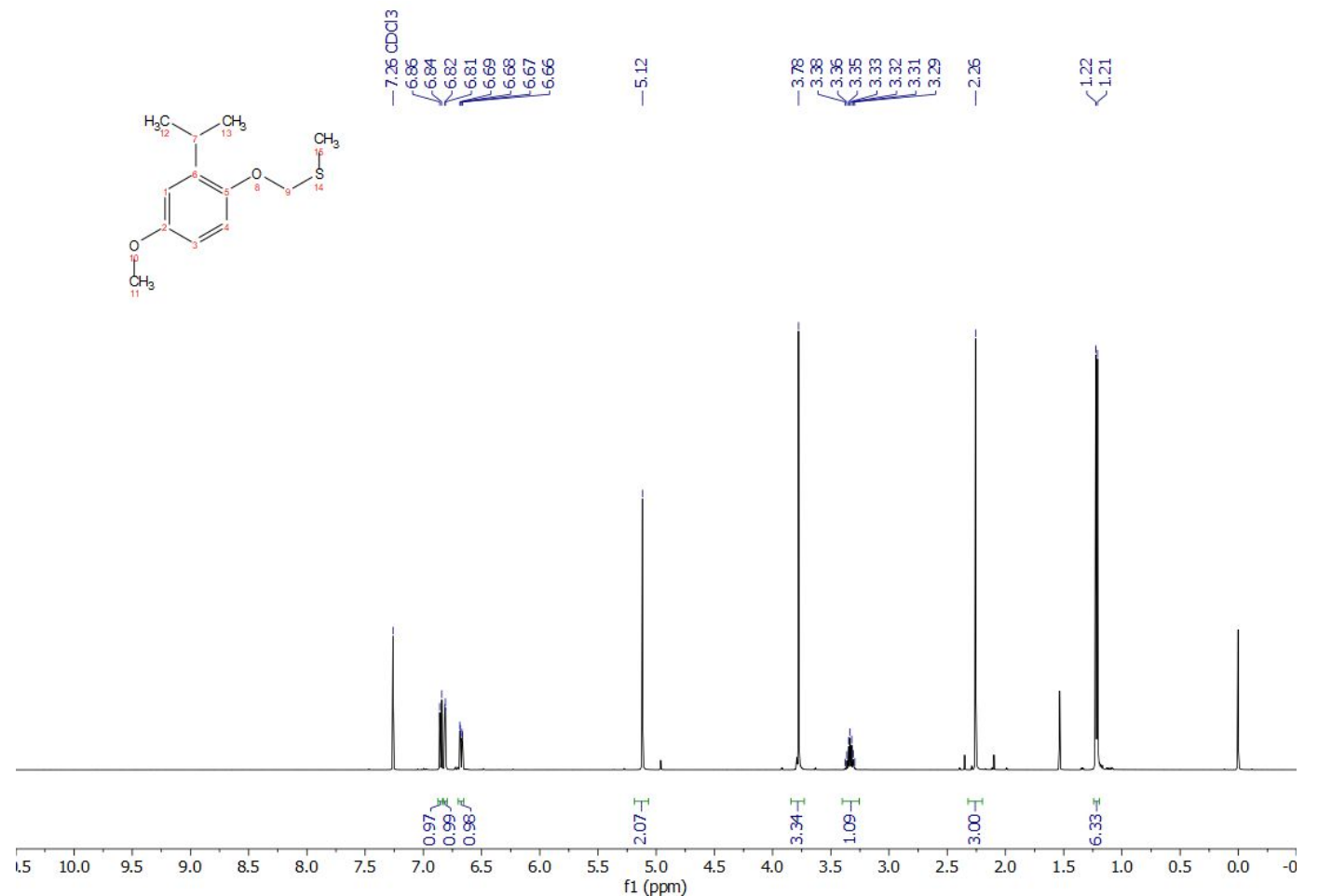

Figure S15. ${ }^{1} \mathrm{H}$ NMR (500 MHz) spectrum of thiomethylether intermediate to ${ }^{15} \mathrm{~N}-2$ in $\mathrm{CDCl}_{3}$.
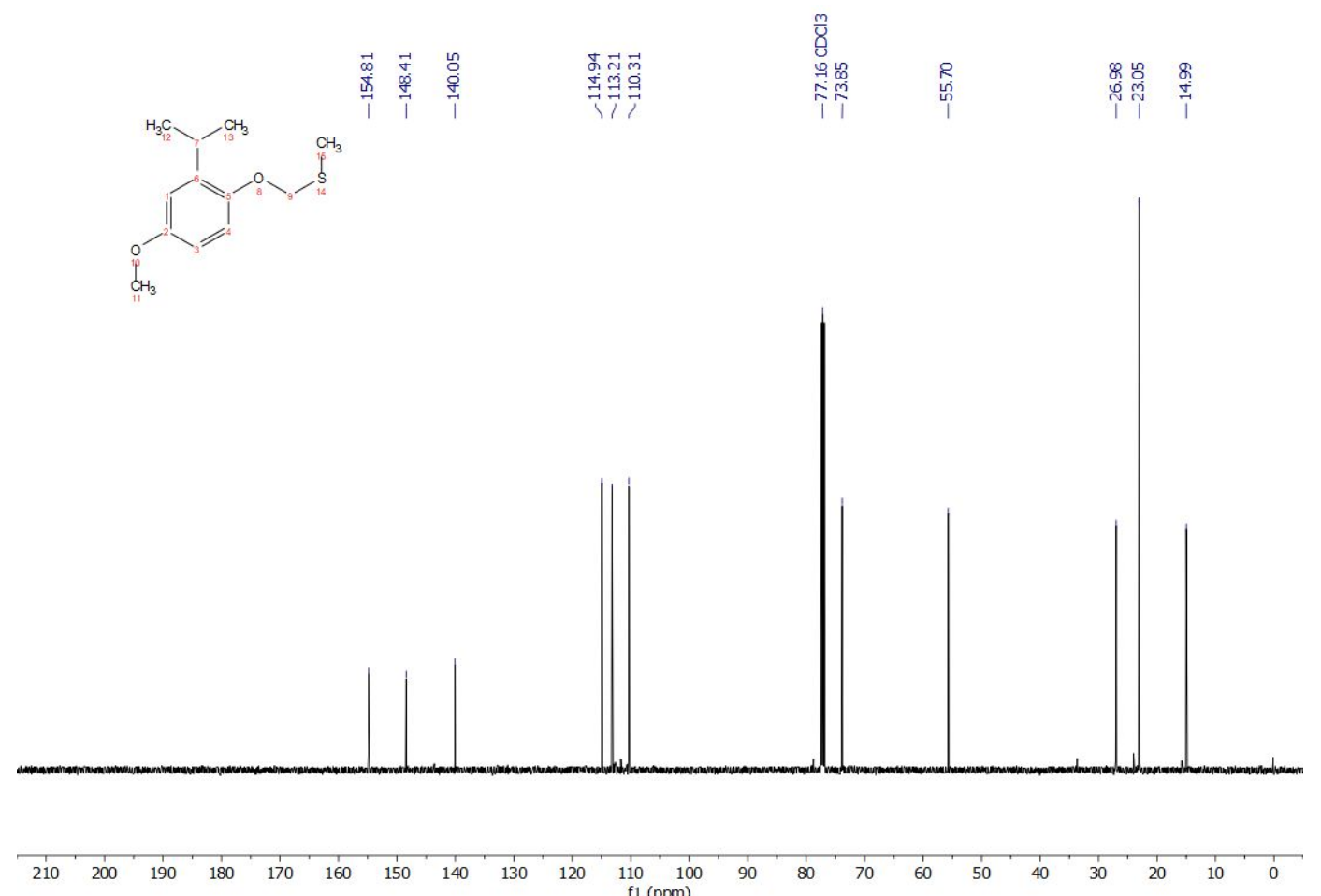

Figure S16. ${ }^{13} \mathrm{C}$ NMR (500 MHz) spectrum of thiomethylether intermediate to ${ }^{15} \mathrm{~N}-2$ in $\mathrm{CDCl}_{3}$. 


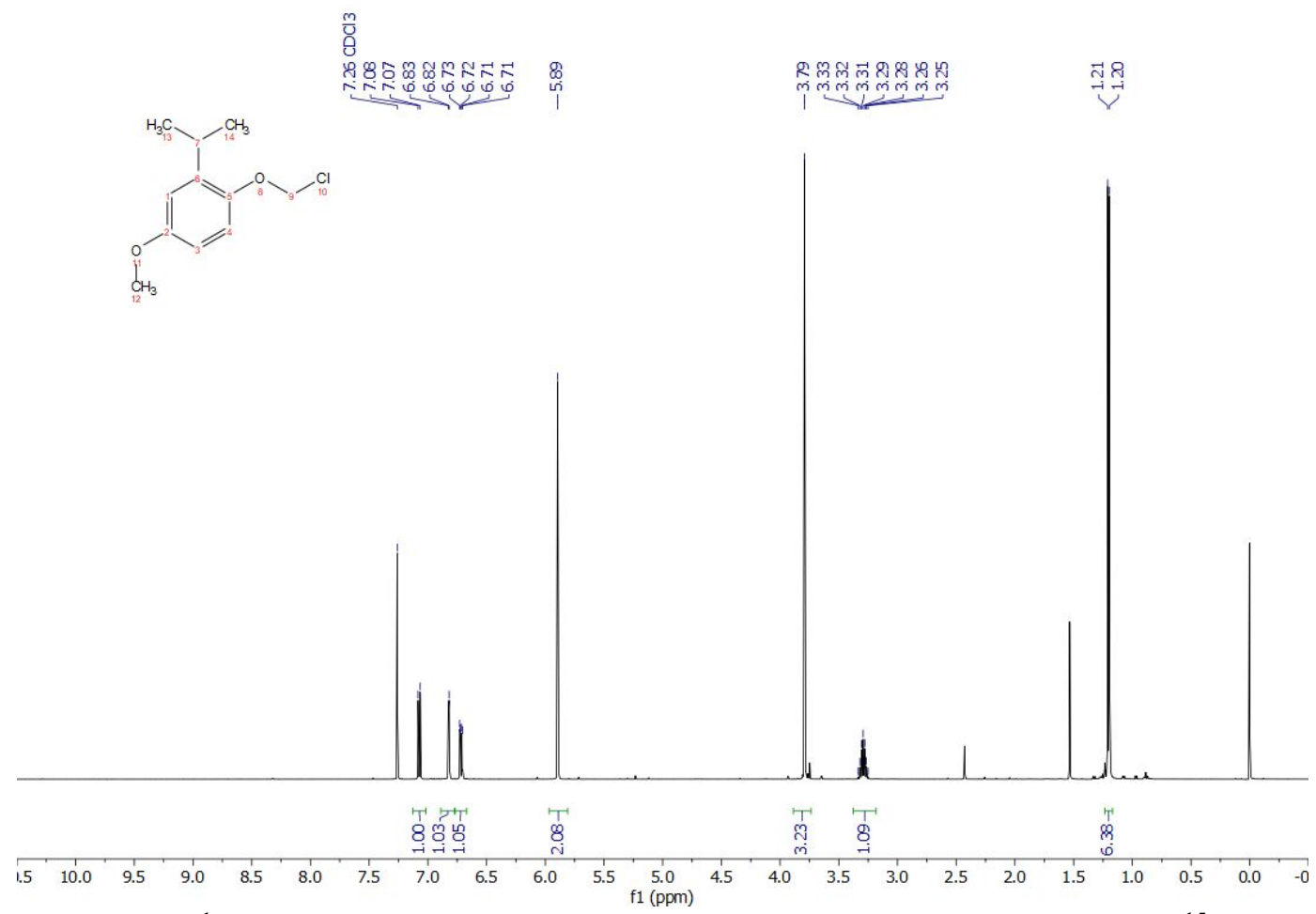

Figure S17. ${ }^{1} \mathrm{H}$ NMR (500 MHz) spectrum of chloromethylether intermediate to ${ }^{15} \mathrm{~N}-2$ in in $\mathrm{CDCl}_{3}$.

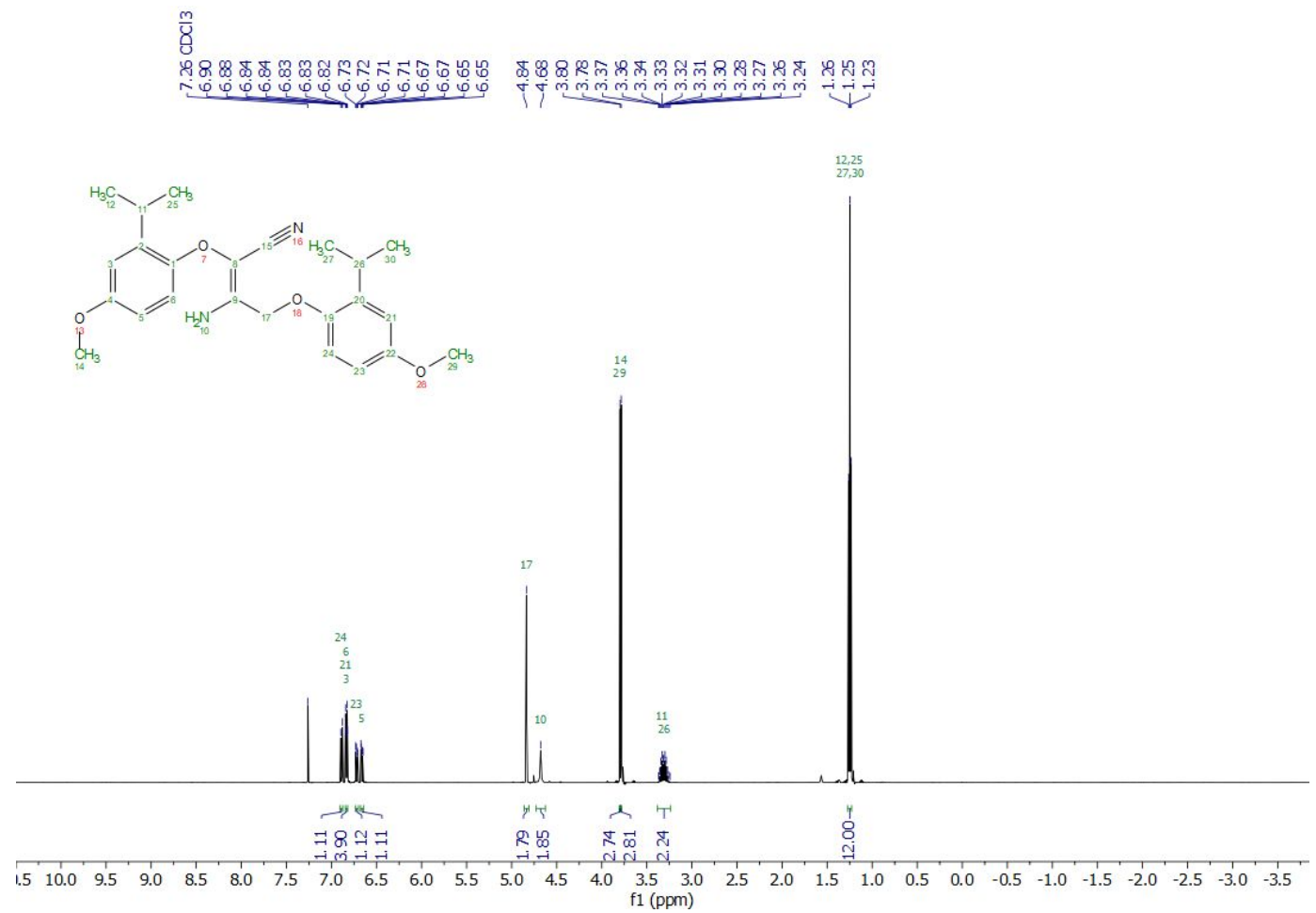

Figure S18. ${ }^{1} \mathrm{H}$ NMR (500 MHz) spectrum of $\mathbf{8 a}$ in $\mathrm{CDCl}_{3}$. 


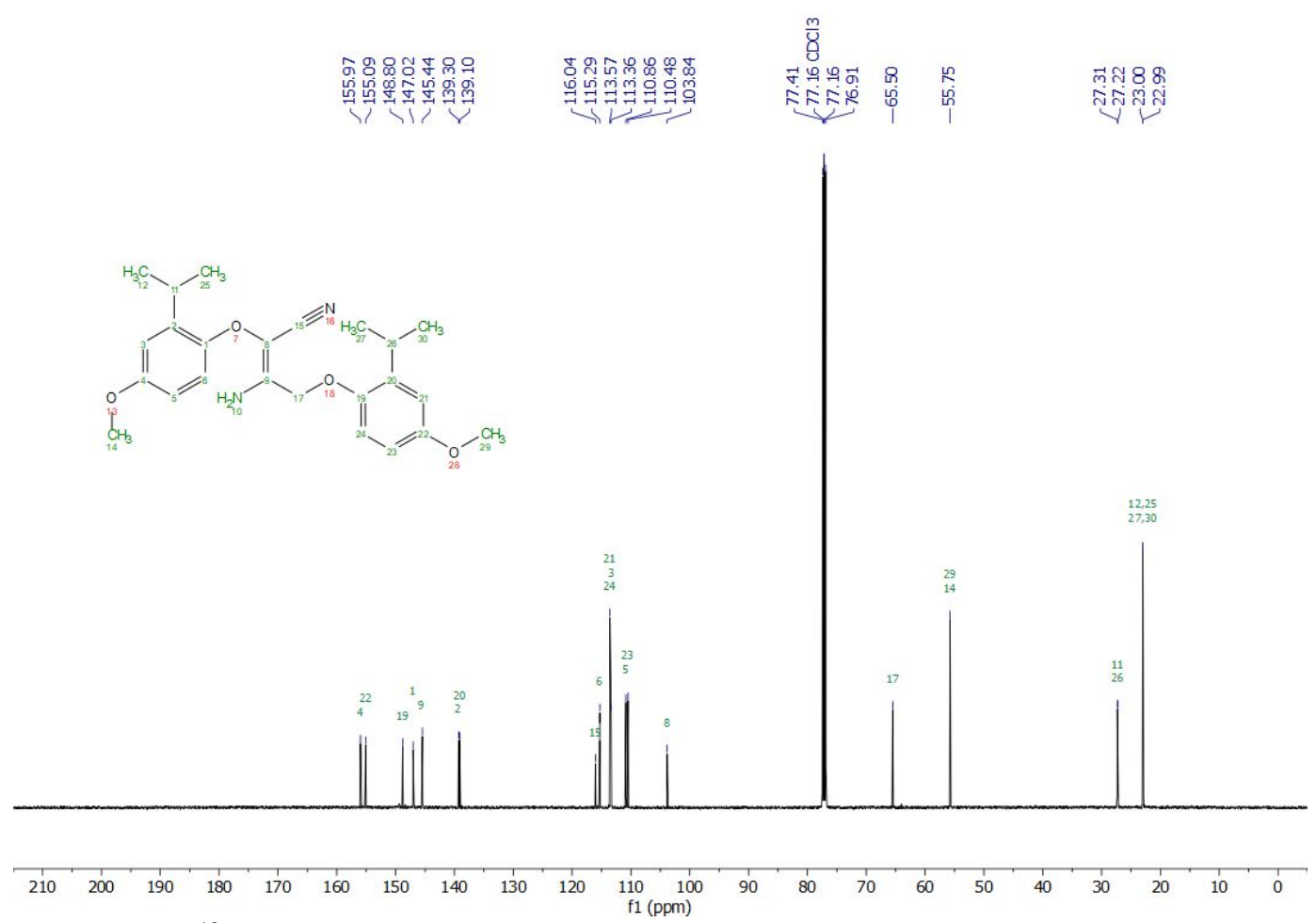

Figure S19. ${ }^{13} \mathrm{C}$ NMR (126 MHz) spectrum of $\mathbf{8 a}$ in $\mathrm{CDCl}_{3}$.

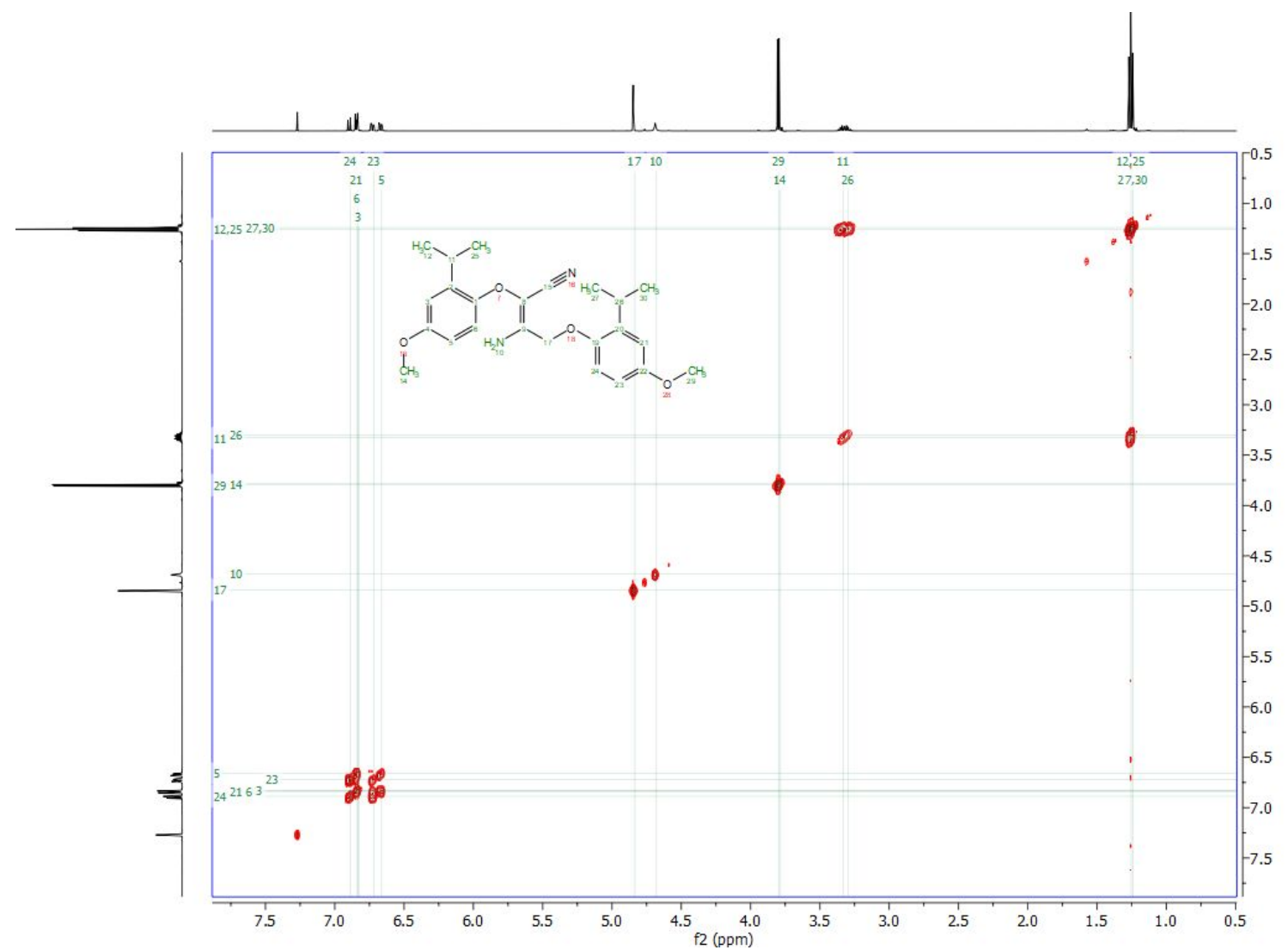

Figure S20. ${ }^{1} \mathrm{H}-{ }^{1} \mathrm{H}$ gCOSY NMR (500 MHz) spectrum of $\mathbf{8 a}$ in $\mathrm{CDCl}_{3}$. 


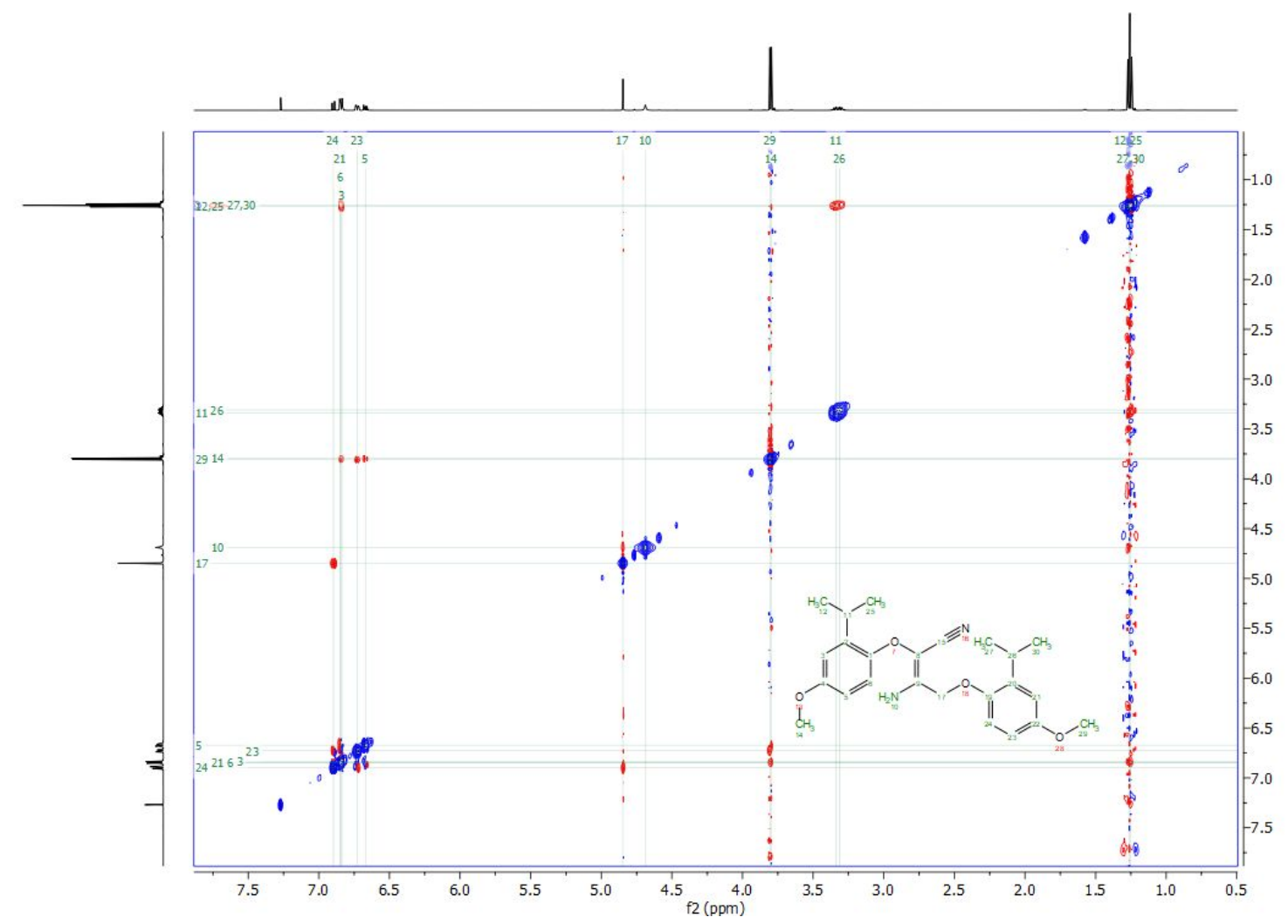

Figure S21. NOESY NMR $(500 \mathrm{MHz}$, mixing time $=500 \mathrm{~ms})$ spectrum of $\mathbf{8 a}$ in $\mathrm{CDCl}_{3}$.

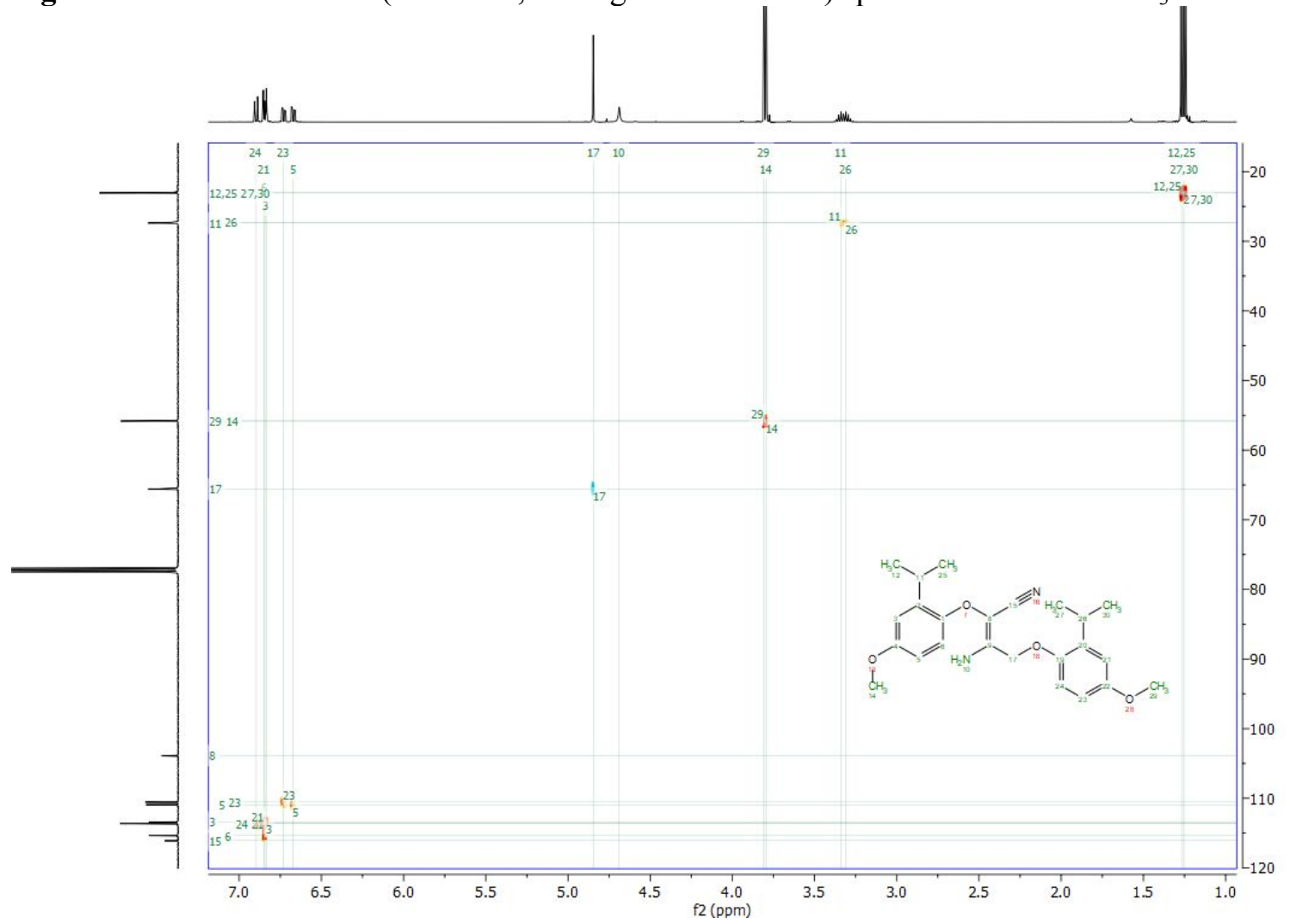

Figure S22. ${ }^{1} \mathrm{H}-{ }^{13} \mathrm{C}$ HSQC NMR $(500 / 126 \mathrm{MHz})$ spectrum of $8 \mathbf{a}$ in $\mathrm{CDCl}_{3}$. 


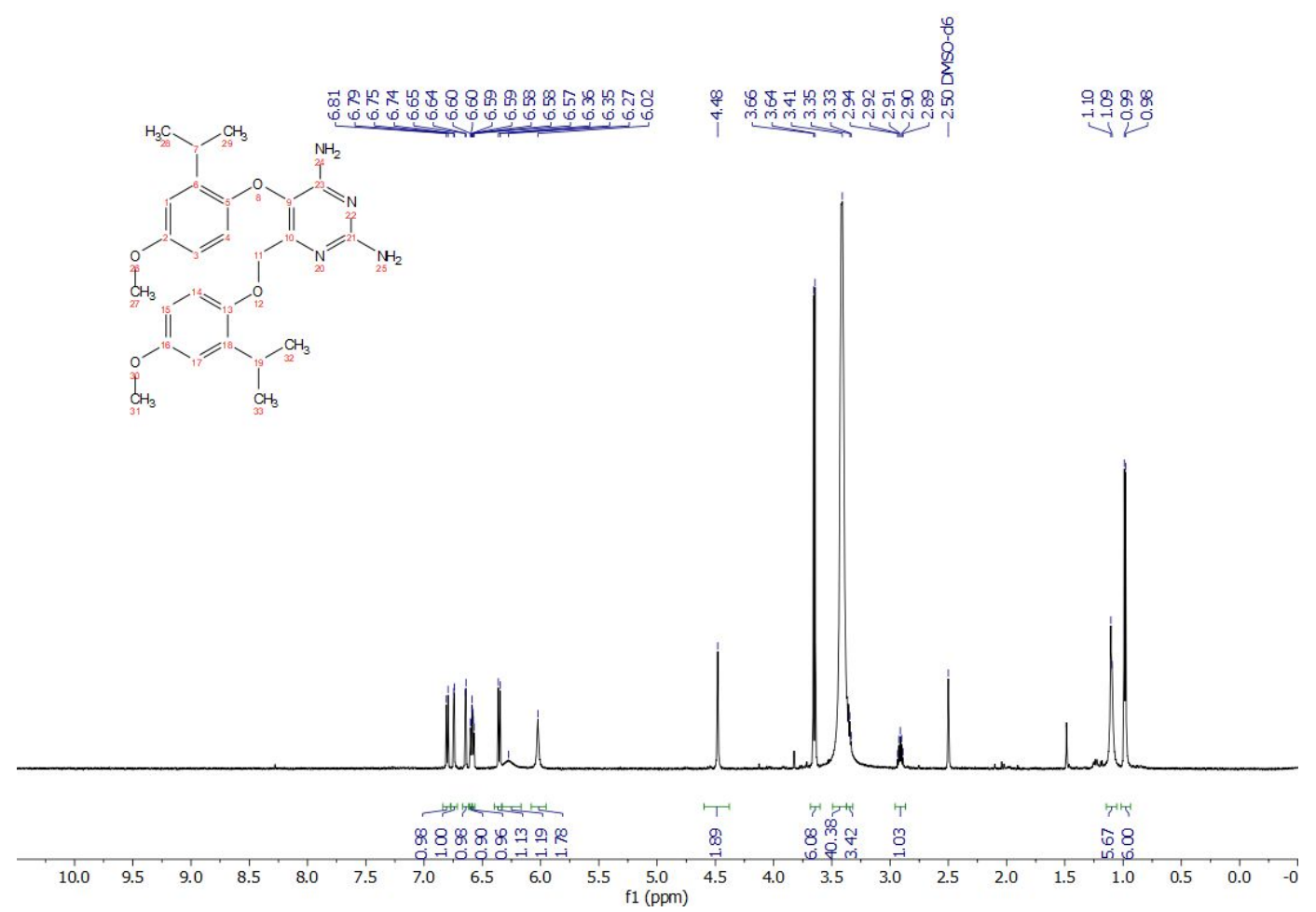

Figure S23. ${ }^{1} \mathrm{H}$ NMR $(500 \mathrm{MHz})$ spectrum of $\mathbf{8 b}$ in $d_{6}$-DMSO.

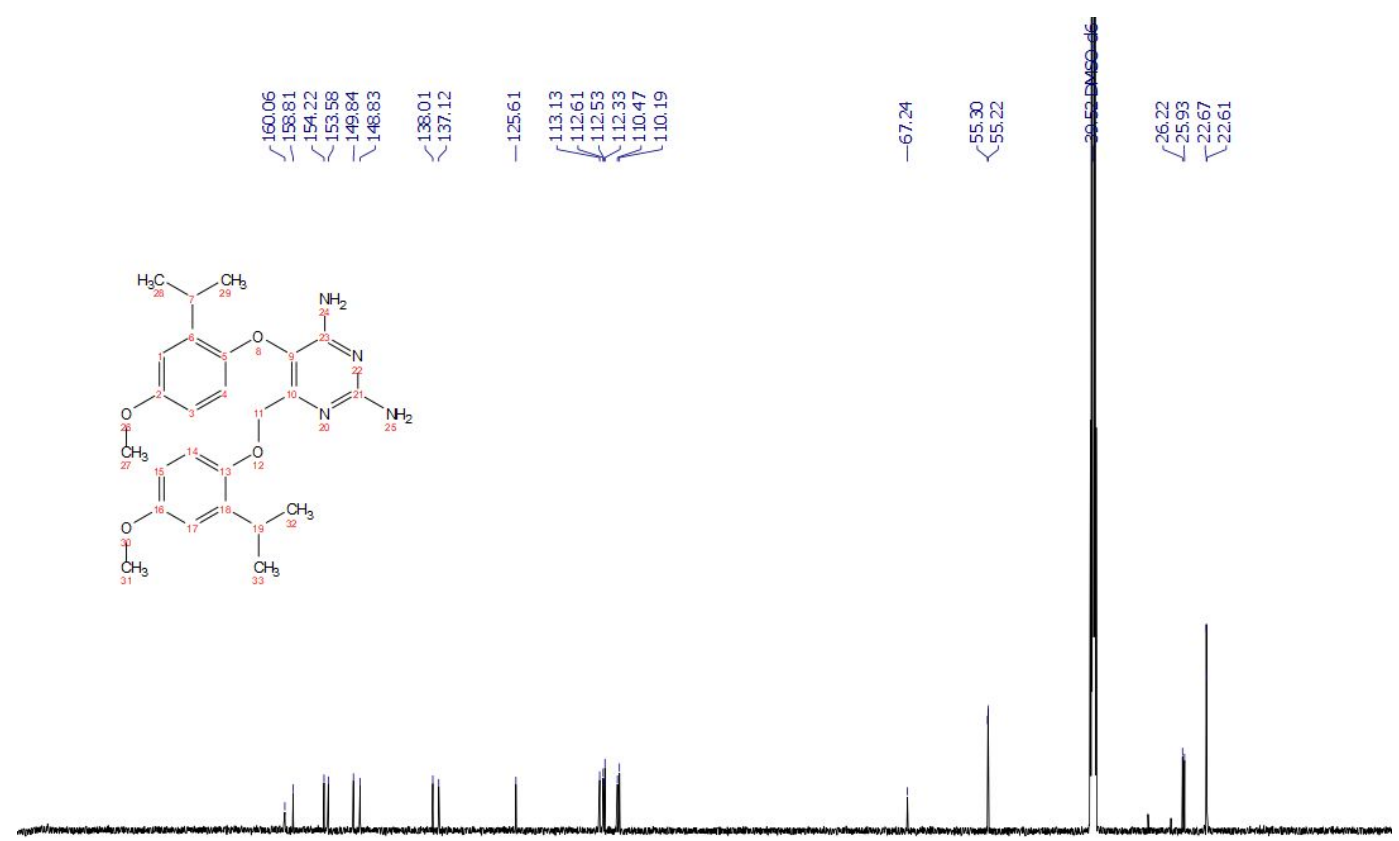

Figure S24. ${ }^{1} \mathrm{H}$ NMR (500 MHz) spectrum of $8 \mathbf{b}$ in $d_{6}$-DMSO. 

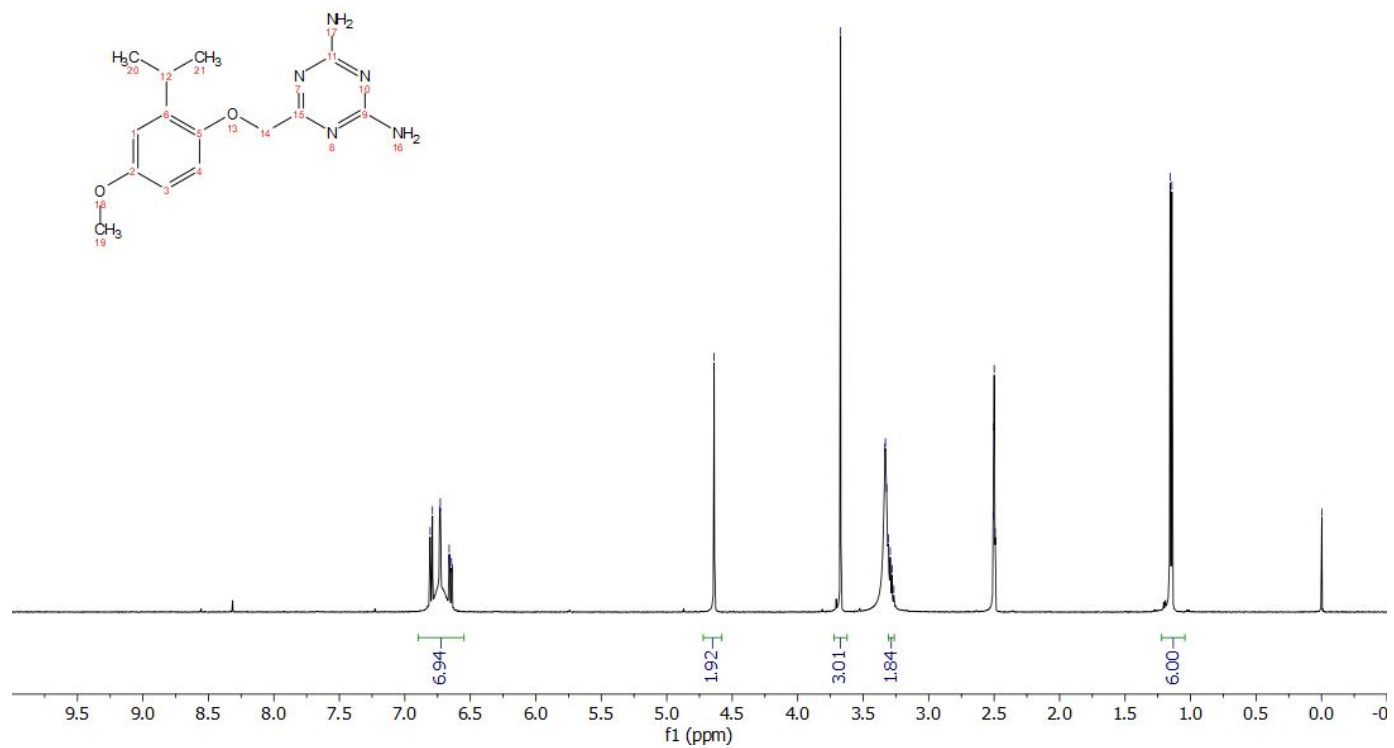

Figure S25. ${ }^{1} \mathrm{H}$ NMR $(500 \mathrm{MHz})$ spectrum of 9 in $d_{6}$-DMSO.

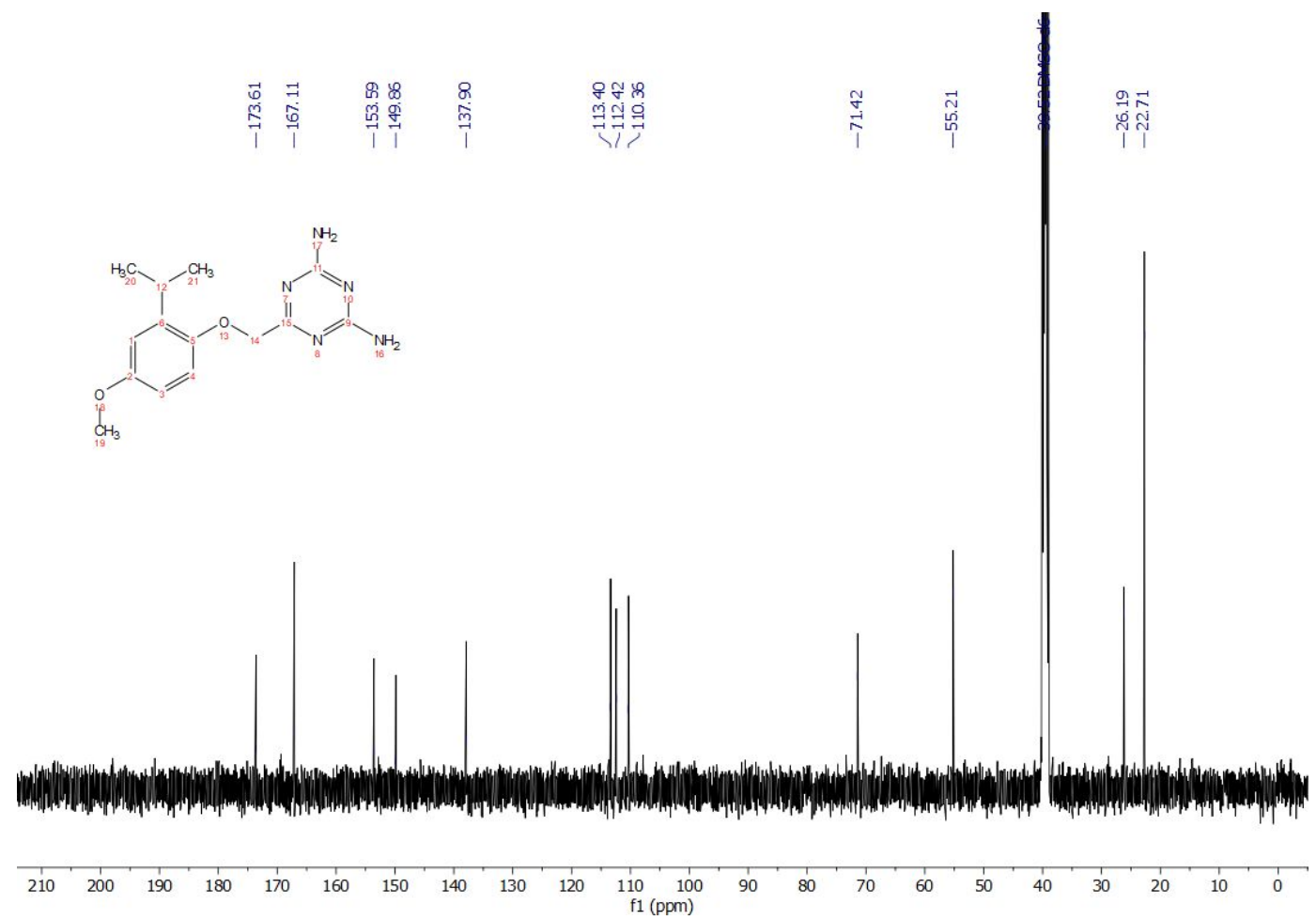

Figure S26. ${ }^{13} \mathrm{C}$ NMR $(126 \mathrm{MHz})$ spectrum of 9 in $d_{6}$-DMSO. 


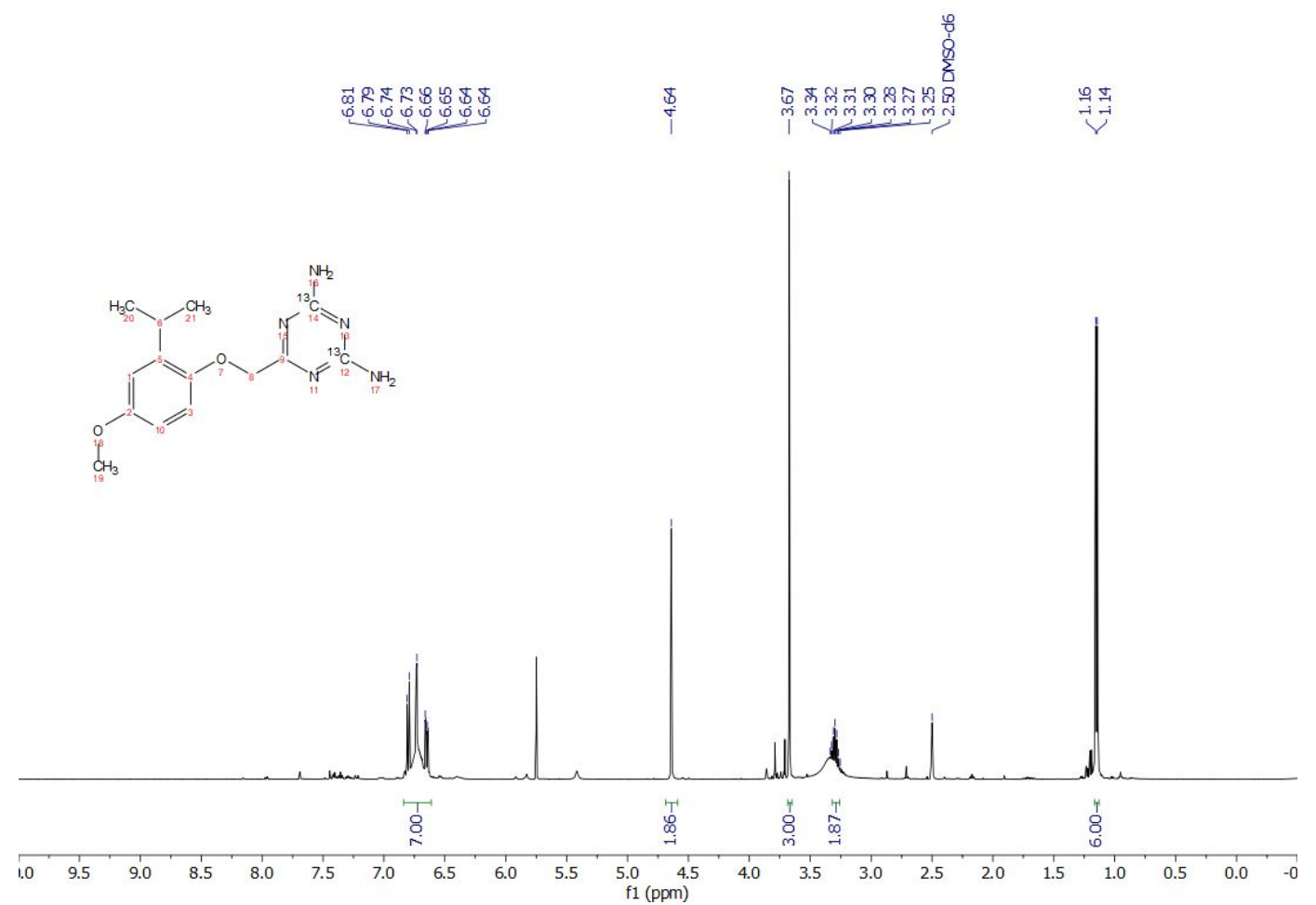

Figure S27. ${ }^{1} \mathrm{H}$ NMR $(500 \mathrm{MHz})$ spectrum of $2,4-{ }^{13} \mathrm{C}_{2}-9$ in $d_{6}$-DMSO.

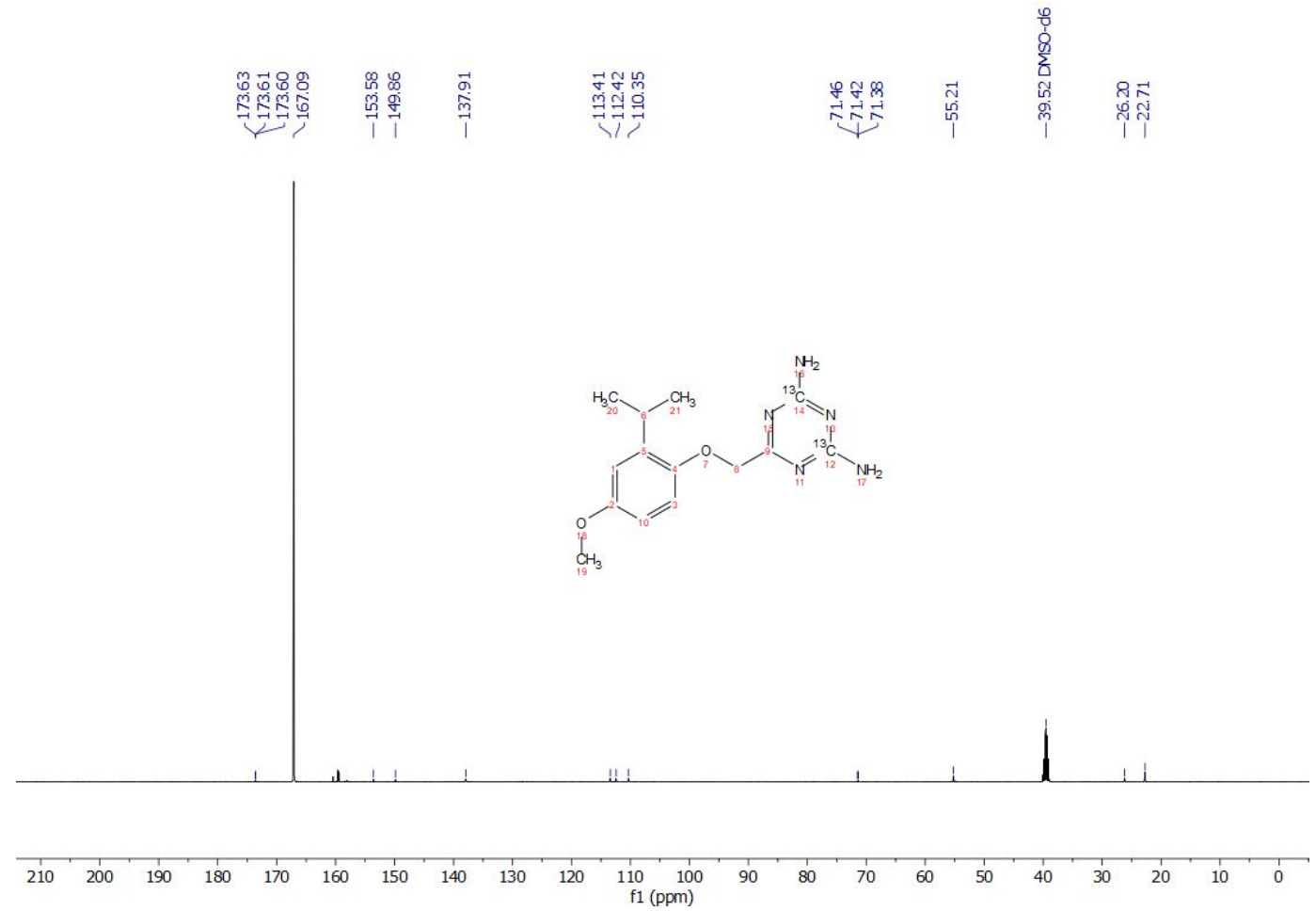

Figure S28. ${ }^{13} \mathrm{C}$ NMR $(126 \mathrm{MHz})$ spectrum of $2,4-{ }^{13} \mathrm{C}_{2}-9$ in $d_{6}$-DMSO. 


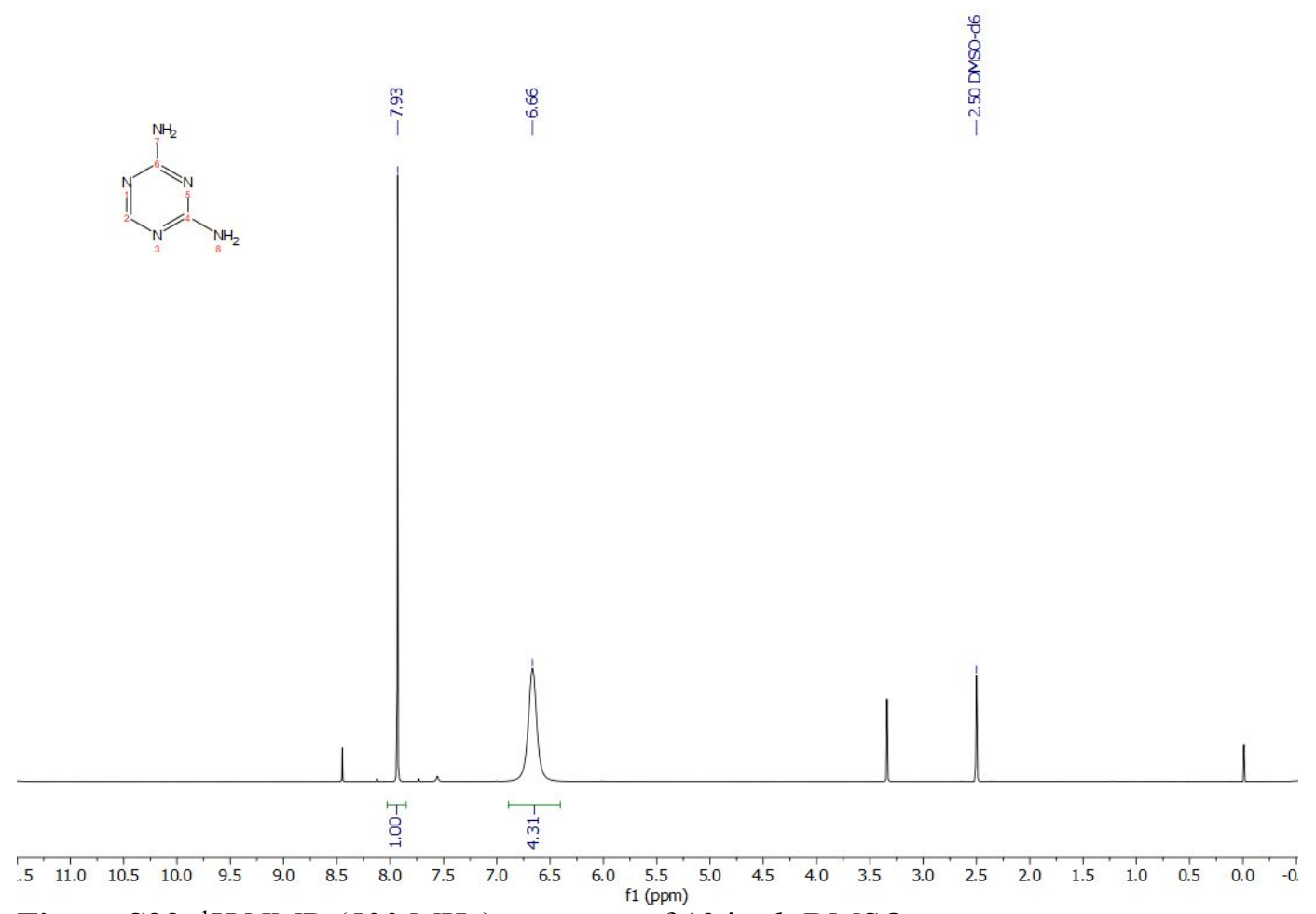

Figure S29. ${ }^{1} \mathrm{H}$ NMR $(500 \mathrm{MHz})$ spectrum of 10 in $d_{6}$-DMSO.

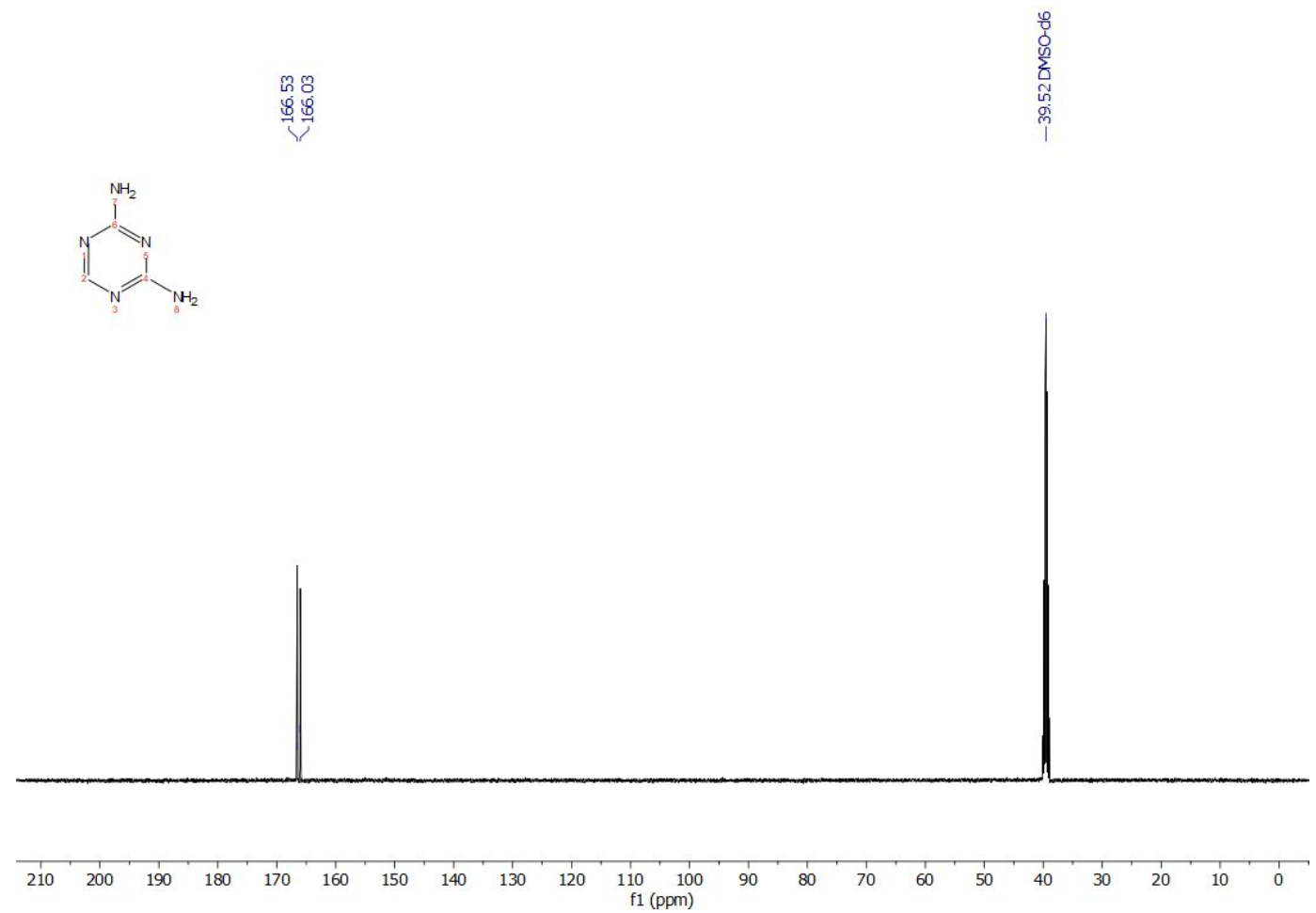

Figure S30. ${ }^{13} \mathrm{C}$ NMR $(126 \mathrm{MHz})$ spectrum of $\mathbf{1 0}$ in $d_{6}$-DMSO. 

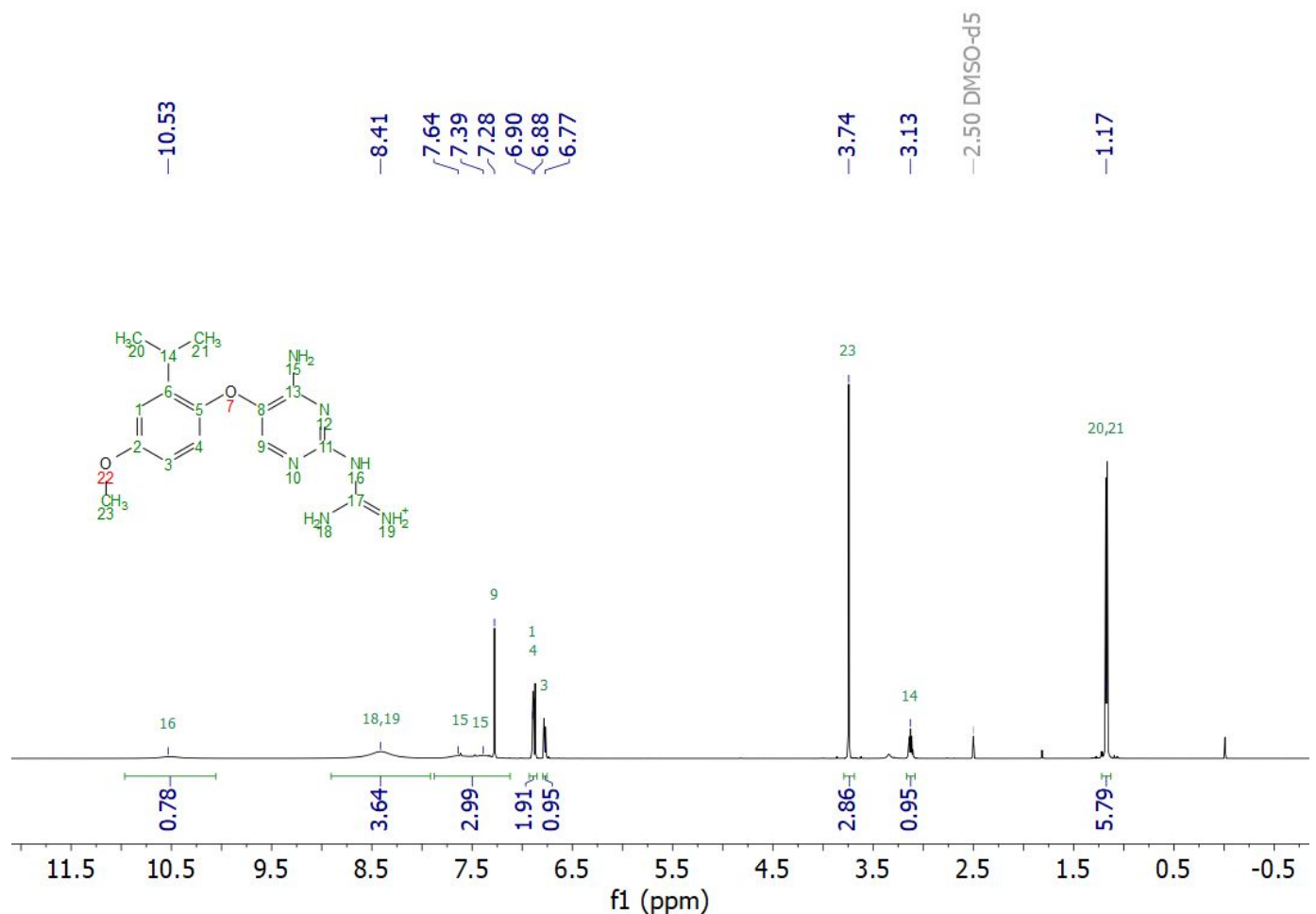

Figure S31. ${ }^{1} \mathrm{H}$ NMR (600 MHz) spectrum of 11 in $d_{6}$-DMSO.
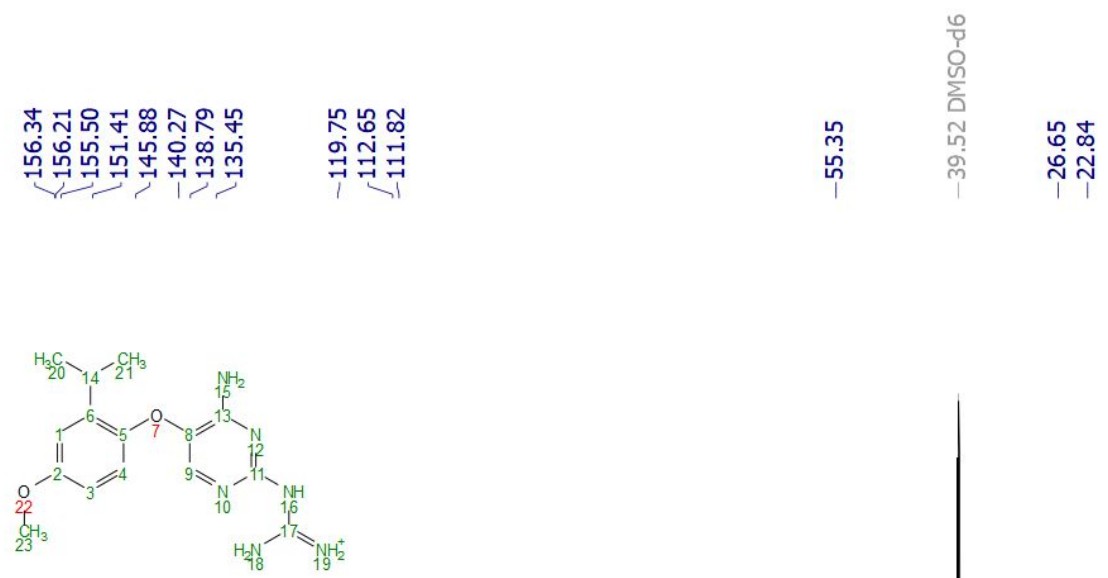

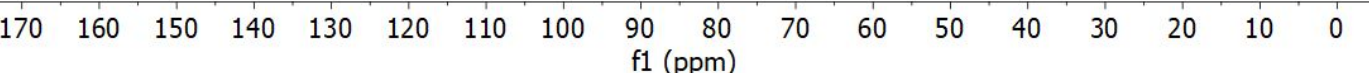

Figure S32. ${ }^{13} \mathrm{C}$ NMR $(151 \mathrm{MHz})$ spectrum of 11 in $d_{6}$-DMSO. 


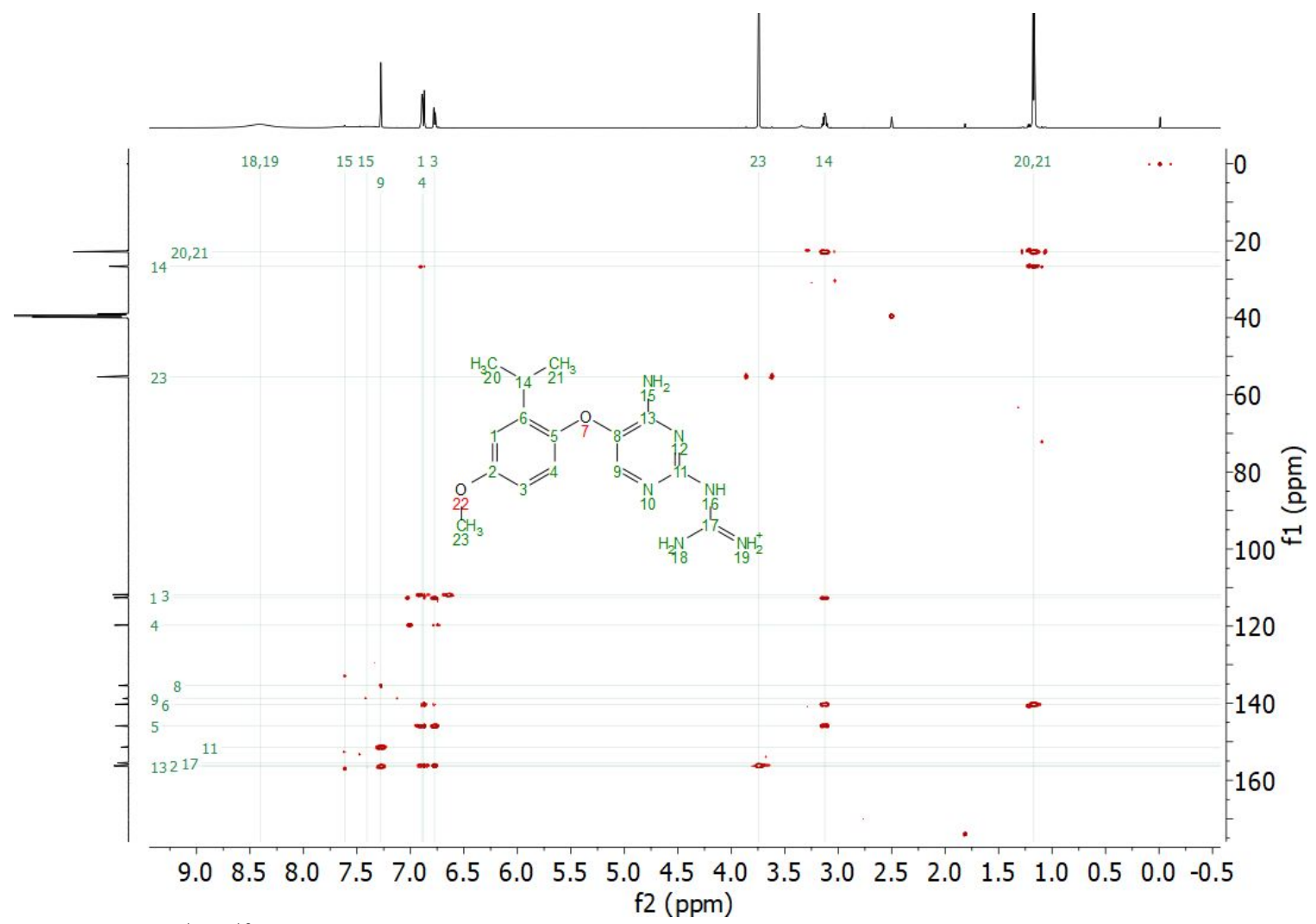

Figure S33. ${ }^{1} \mathrm{H}^{-13} \mathrm{C}$ HMBC NMR $(600 / 151 \mathrm{MHz})$ spectrum of 11 in $d_{\sigma}$-DMSO.

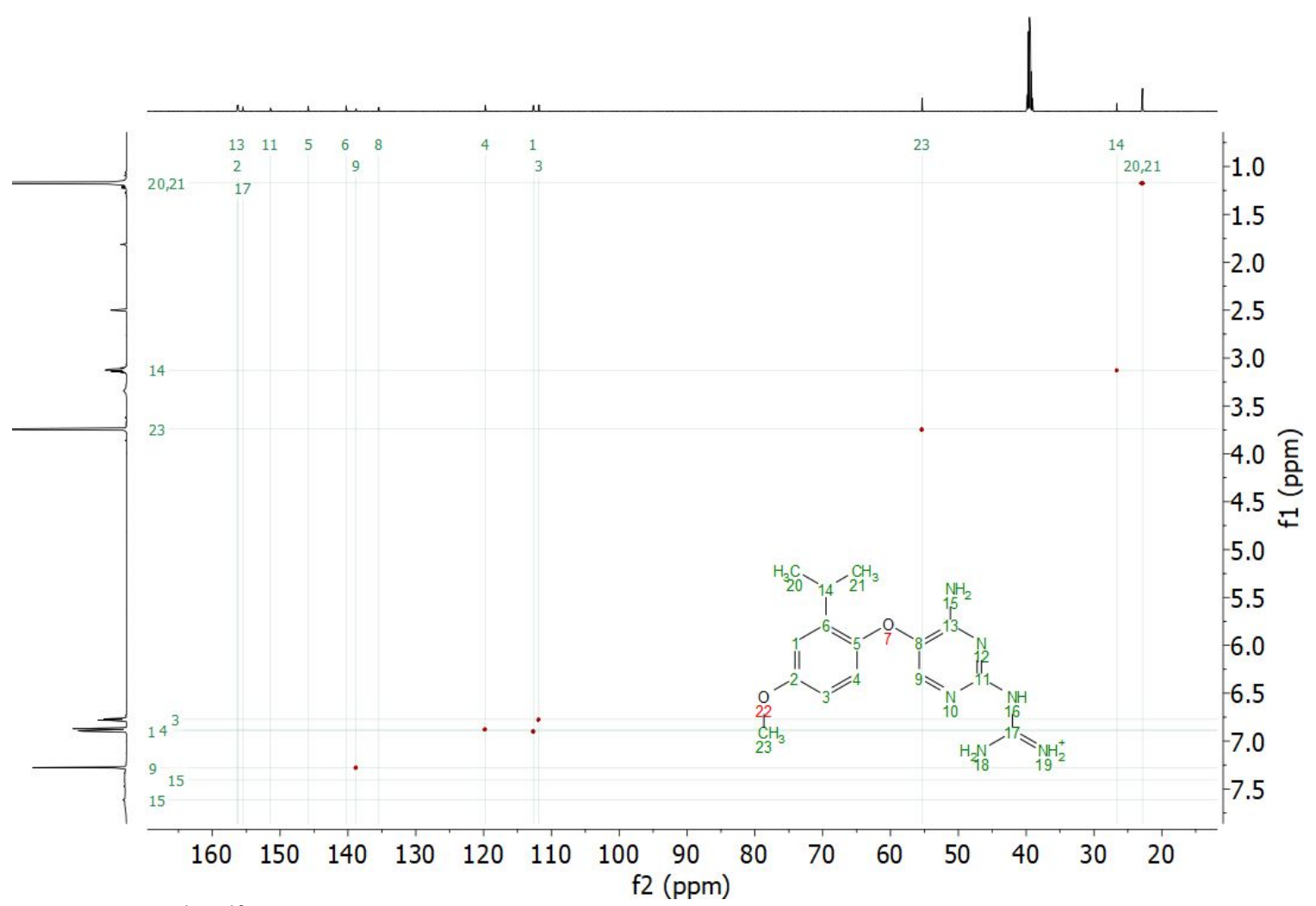

Figure S34. ${ }^{1} \mathrm{H}-{ }^{13} \mathrm{C}$ HETCOR NMR $(600 / 151 \mathrm{MHz})$ spectrum of 11 in $d_{6}$-DMSO. 


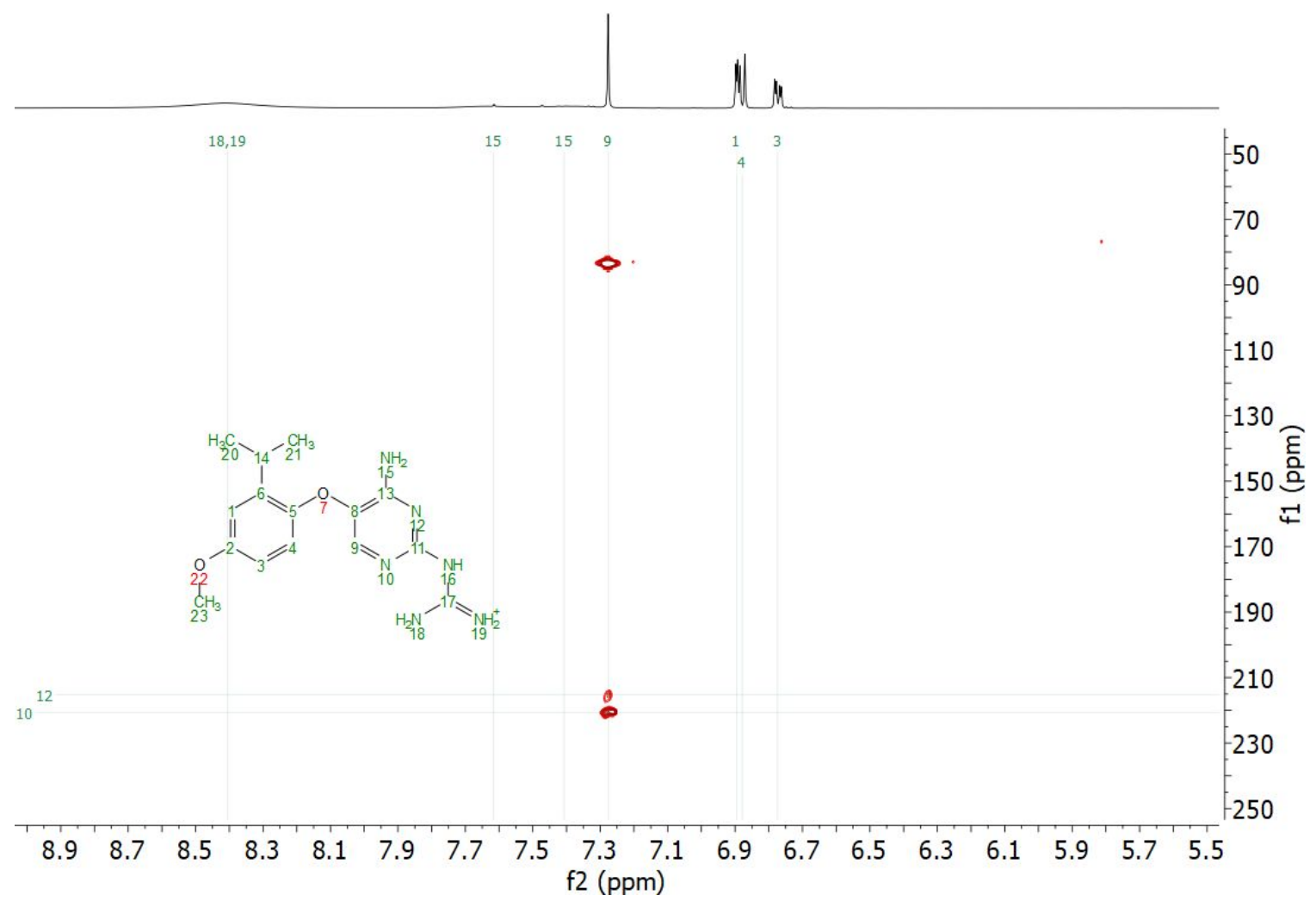

Figure S35. ${ }^{1} \mathrm{H}^{-15} \mathrm{~N}$ HMBC NMR $(600 / 60 \mathrm{MHz})$ spectrum of 11 in $d_{6}$-DMSO.

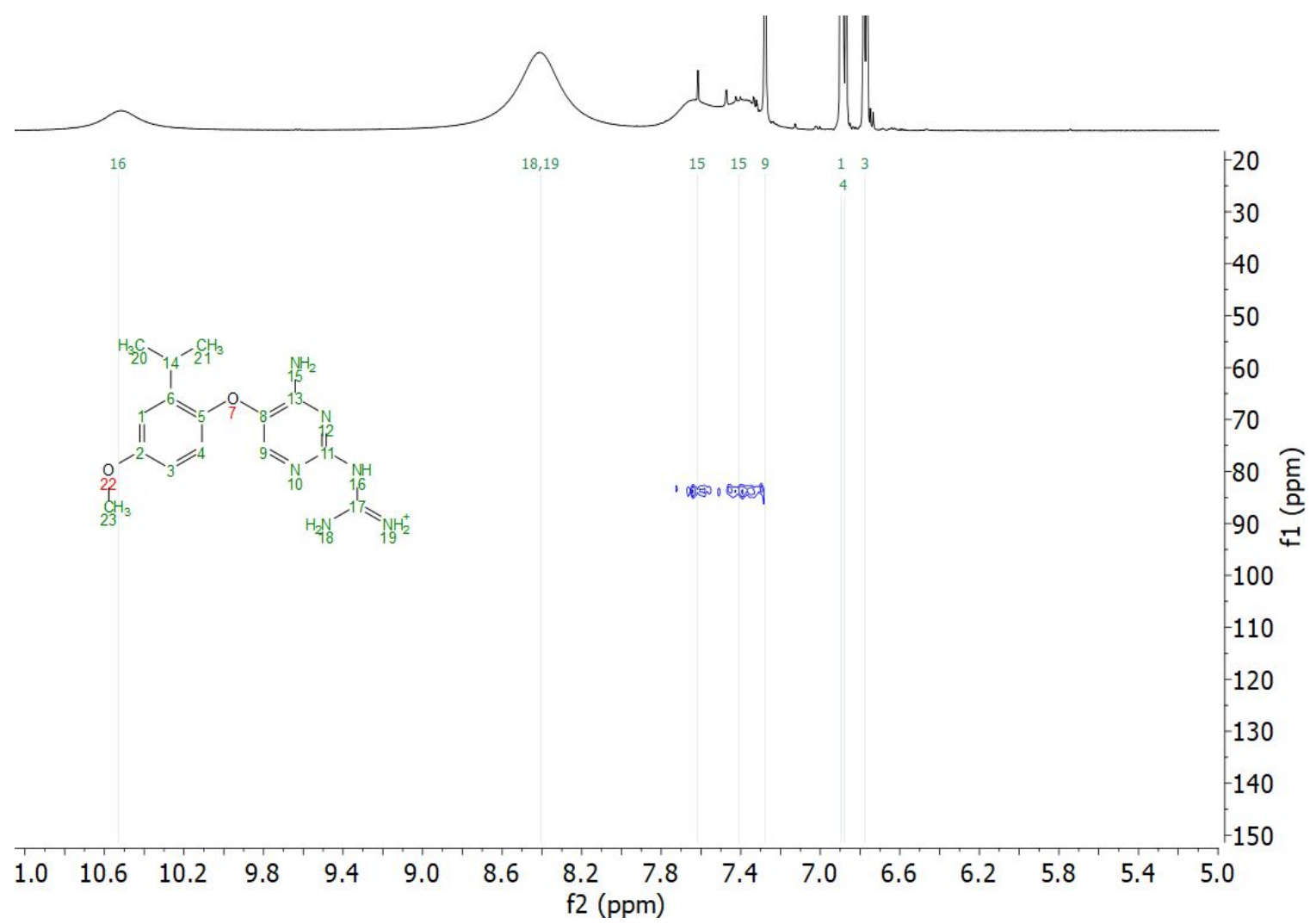

Figure S36. ${ }^{1} \mathrm{H}^{-15} \mathrm{~N}$ HSQC NMR $(600 / 60 \mathrm{MHz})$ spectrum of 11 in $d_{\sigma}$-DMSO. 


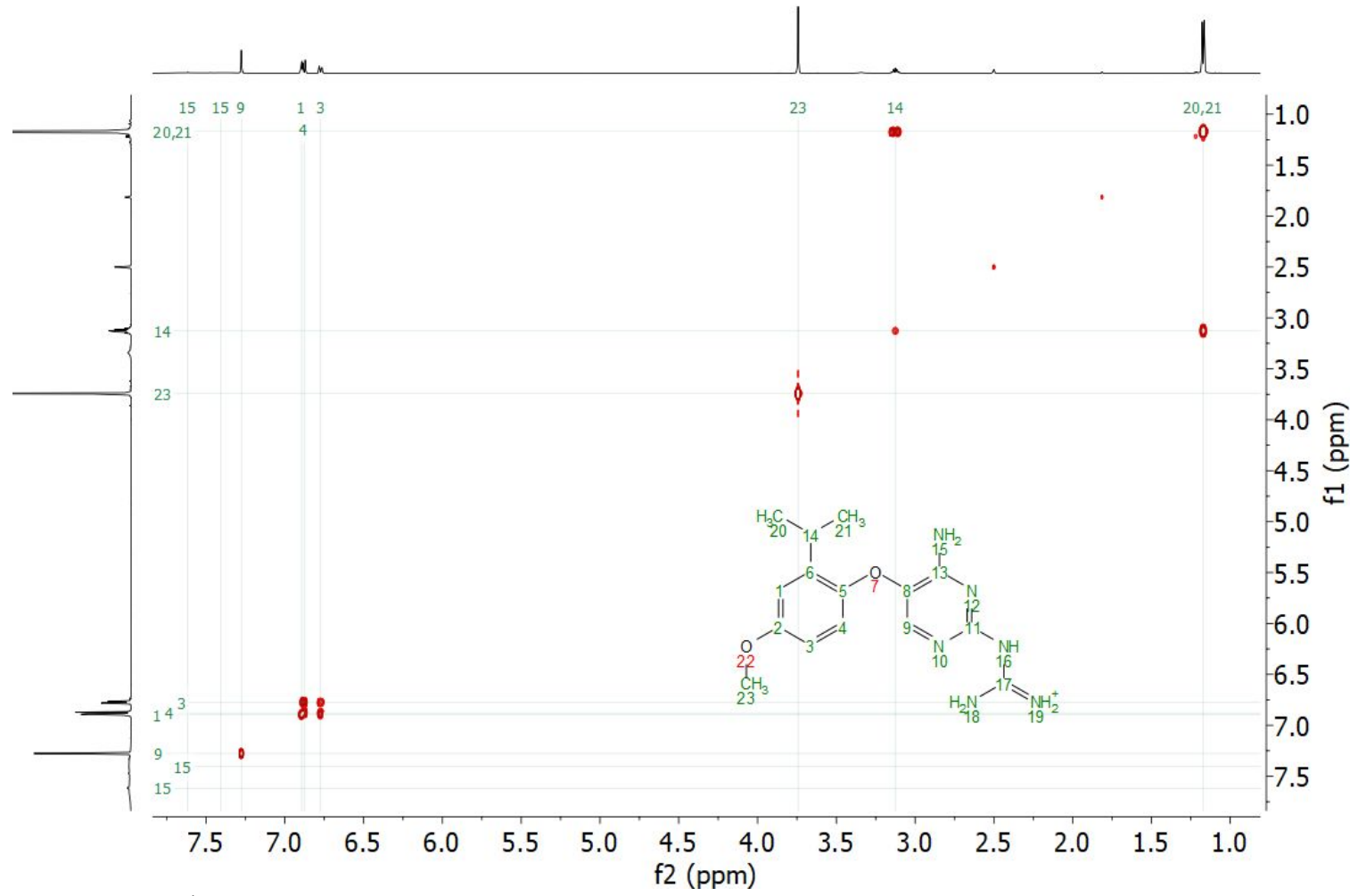

Figure S37. ${ }^{1} \mathrm{H}$ COSY NMR (600 MHz) spectrum of 11 in $d_{6}$-DMSO.

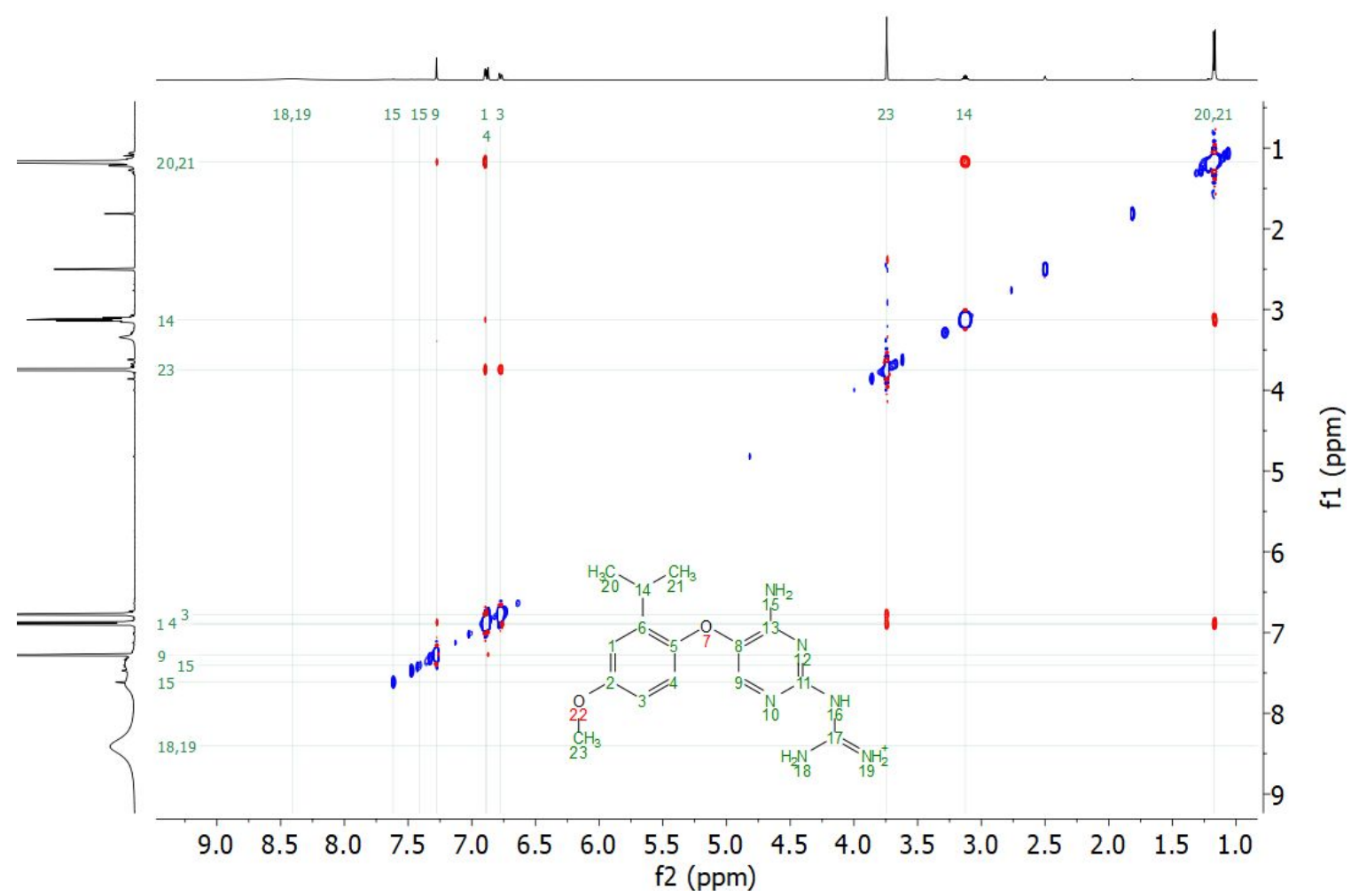

Figure S38. ${ }^{1} \mathrm{H}$ ROESY NMR $(600 \mathrm{MHz}$, mixing time $=200 \mathrm{~ms})$ spectrum of 11 in $d_{\sigma}$-DMSO. 


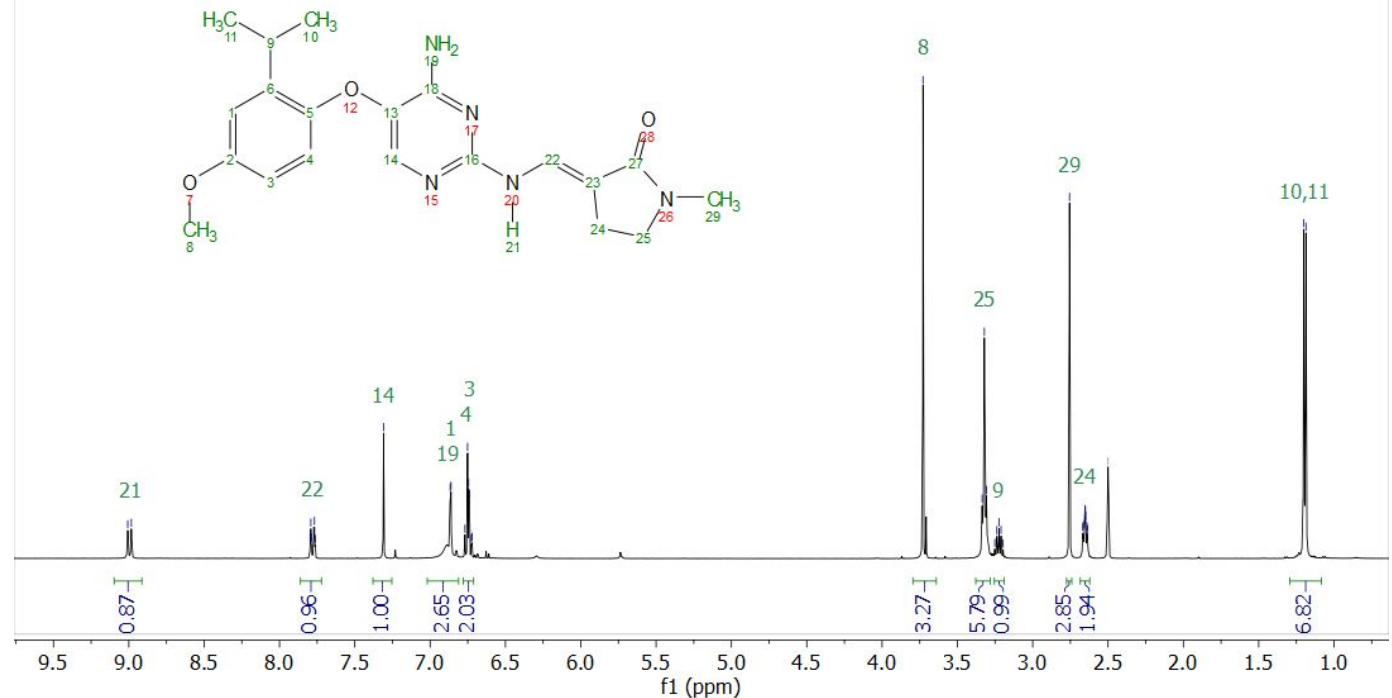

Figure S39. ${ }^{1} \mathrm{H}$ NMR $(500 \mathrm{MHz})$ spectrum of 14a in $d_{6}$-DMSO.

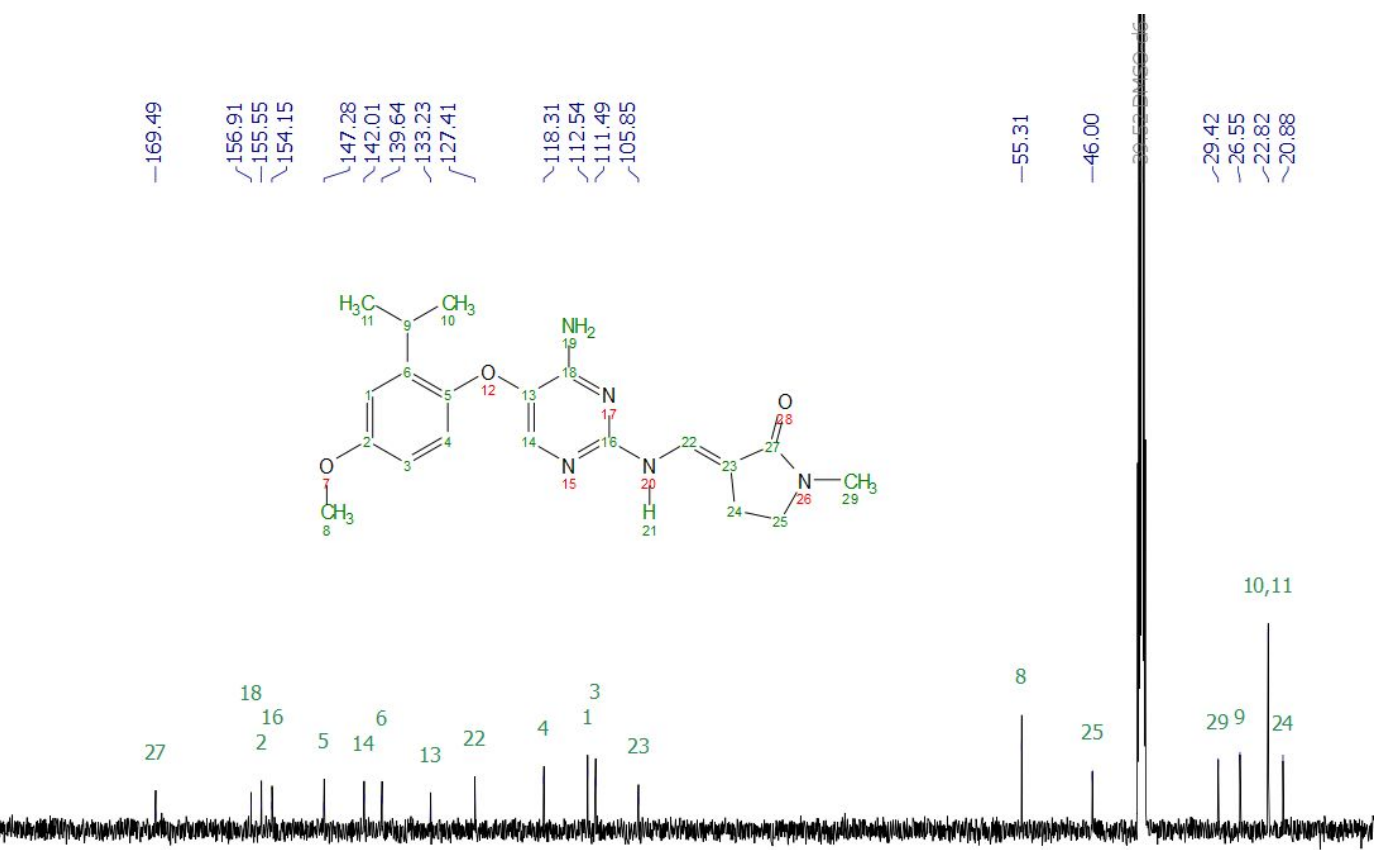

0

Figure S40. ${ }^{13} \mathrm{C}$ NMR (126 MHz) spectrum of 14a in $d_{6}$-DMSO. 


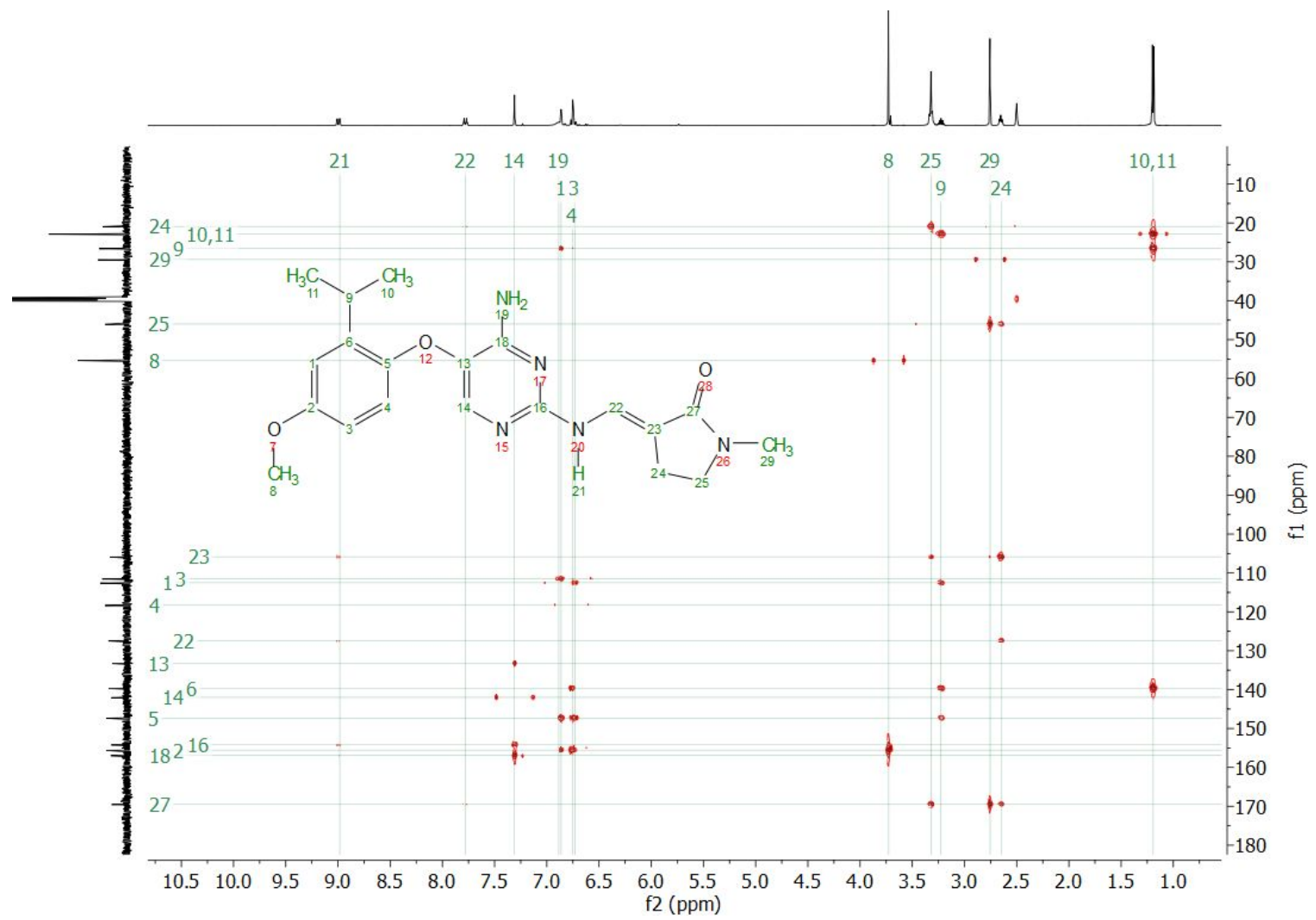

Figure S41. ${ }^{1} \mathrm{H}^{-13} \mathrm{C}$ HMBC NMR $(500 / 126 \mathrm{MHz})$ spectrum of 14a in $d_{\sigma}$-DMSO.

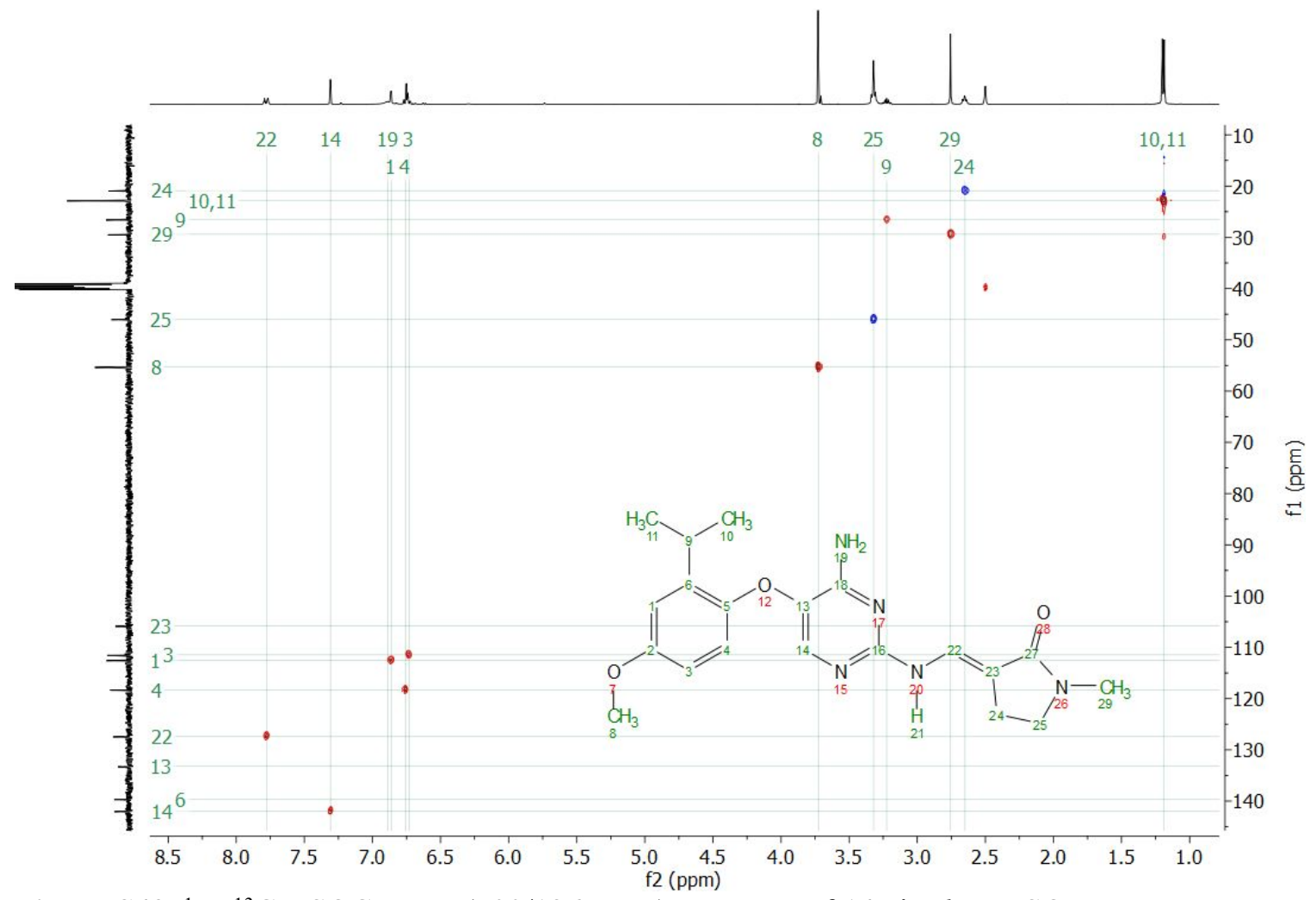

Figure S42. ${ }^{1} \mathrm{H}^{-13} \mathrm{C}$ HSQC NMR $(500 / 126 \mathrm{MHz})$ spectrum of 14a in $d_{6}$-DMSO. 


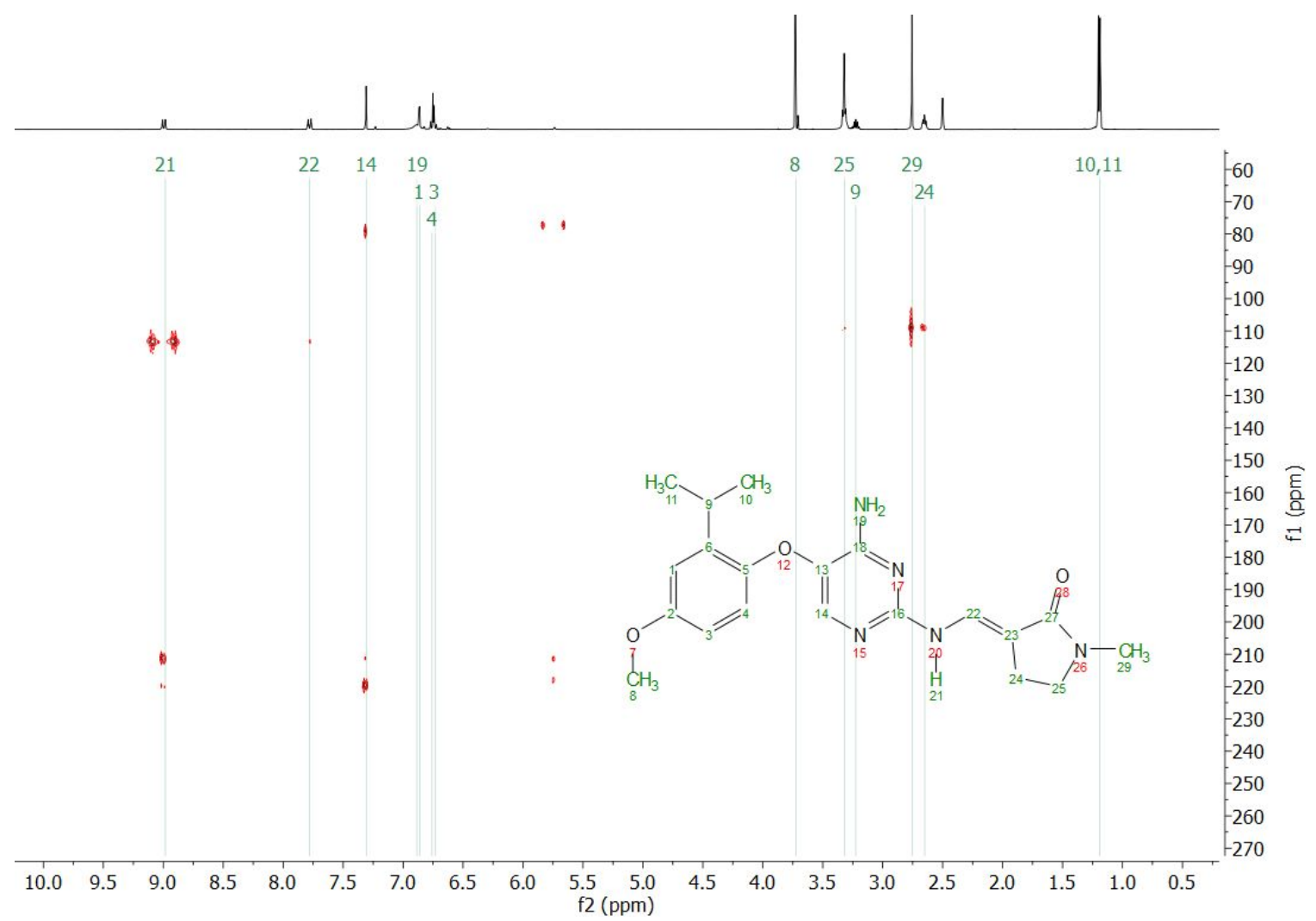

Figure S43. ${ }^{1} \mathrm{H}^{-15} \mathrm{~N}$ HMBC NMR $(500 / 51 \mathrm{MHz})$ spectrum of 14a in $d_{\sigma}$-DMSO.

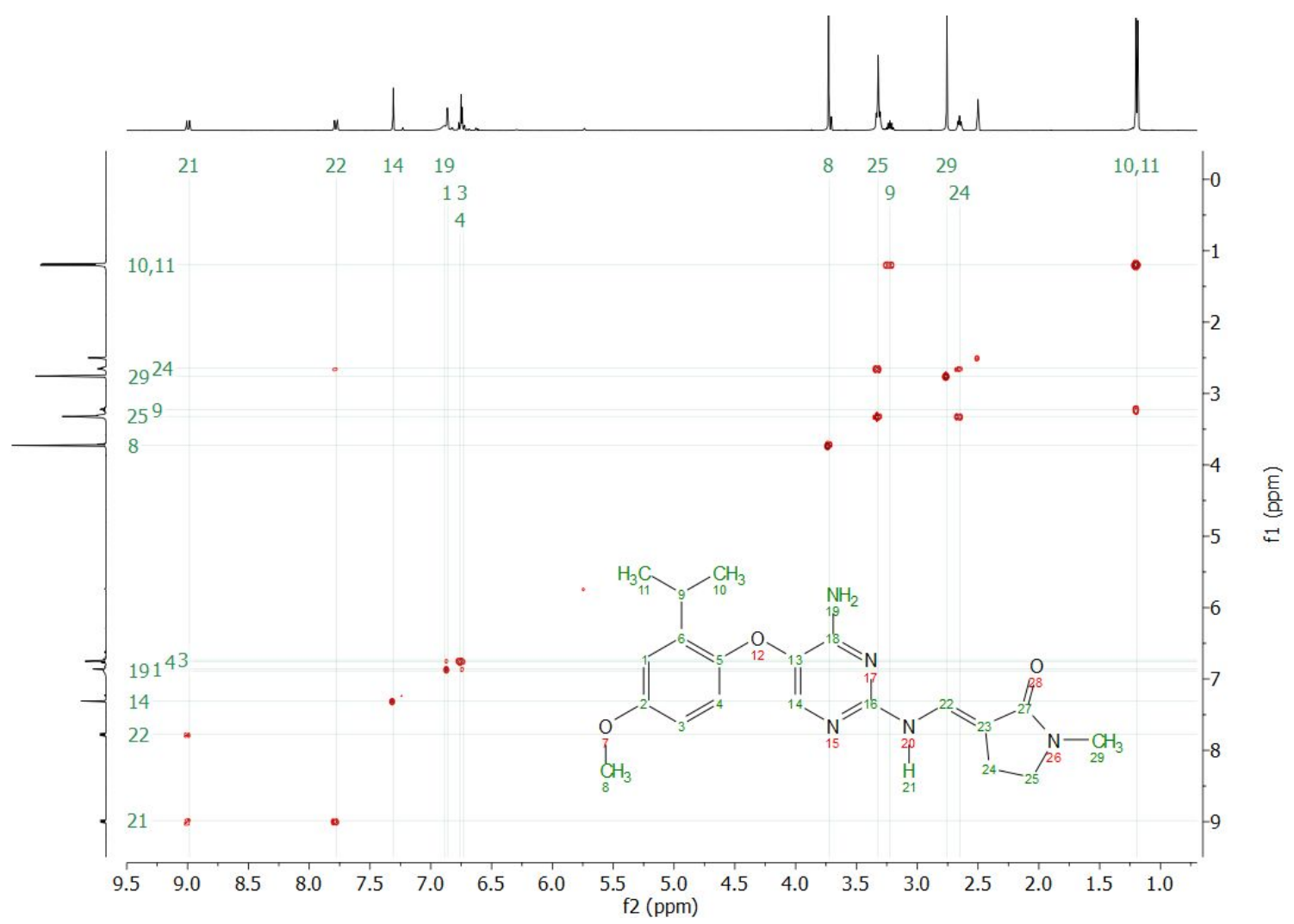

Figure S44. ${ }^{1} \mathrm{H}$ COSY NMR $(500 \mathrm{MHz})$ spectrum of $14 \mathbf{a}$ in $d_{6}$-DMSO. 

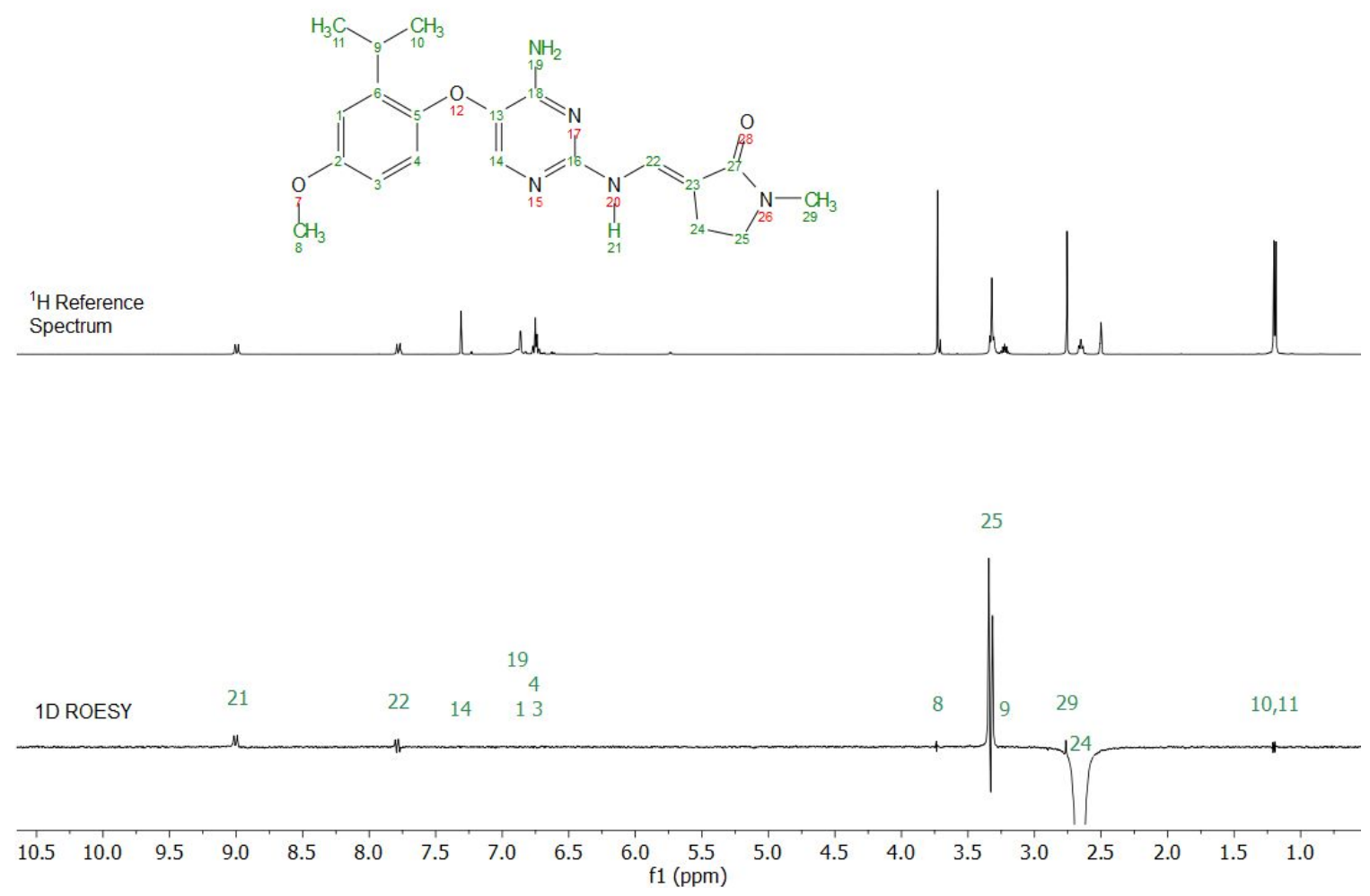

Figure S45. ${ }^{1} \mathrm{H}$ ROESY NMR (500 MHz, mixing time $\left.=200 \mathrm{~ms}\right)$ spectrum of 14a in $d_{\sigma}$-DMSO.

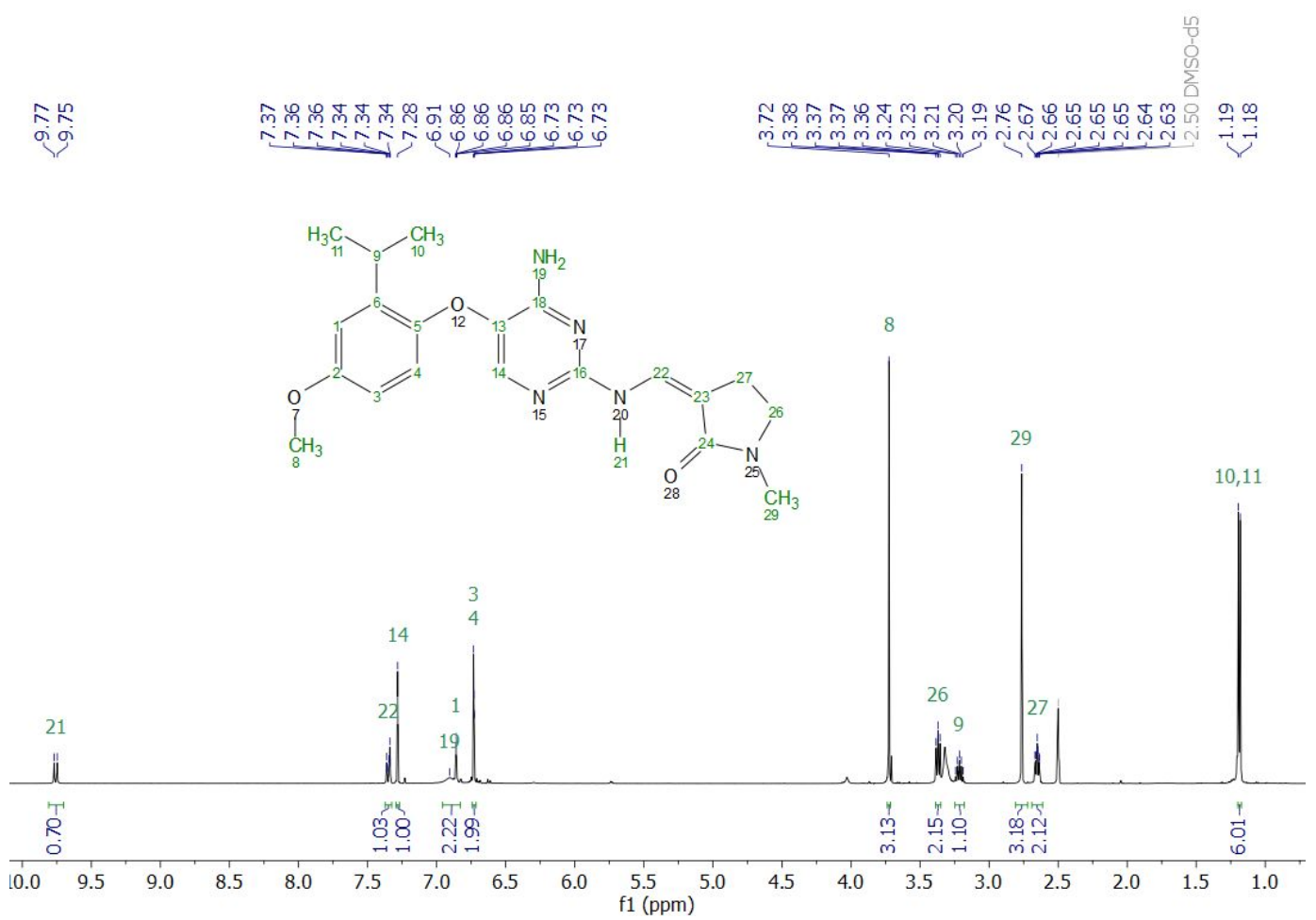

Figure S46. ${ }^{1} \mathrm{H}$ NMR $(500 \mathrm{MHz})$ spectrum of $\mathbf{1 4 b}$ in $d_{6}$-DMSO. 

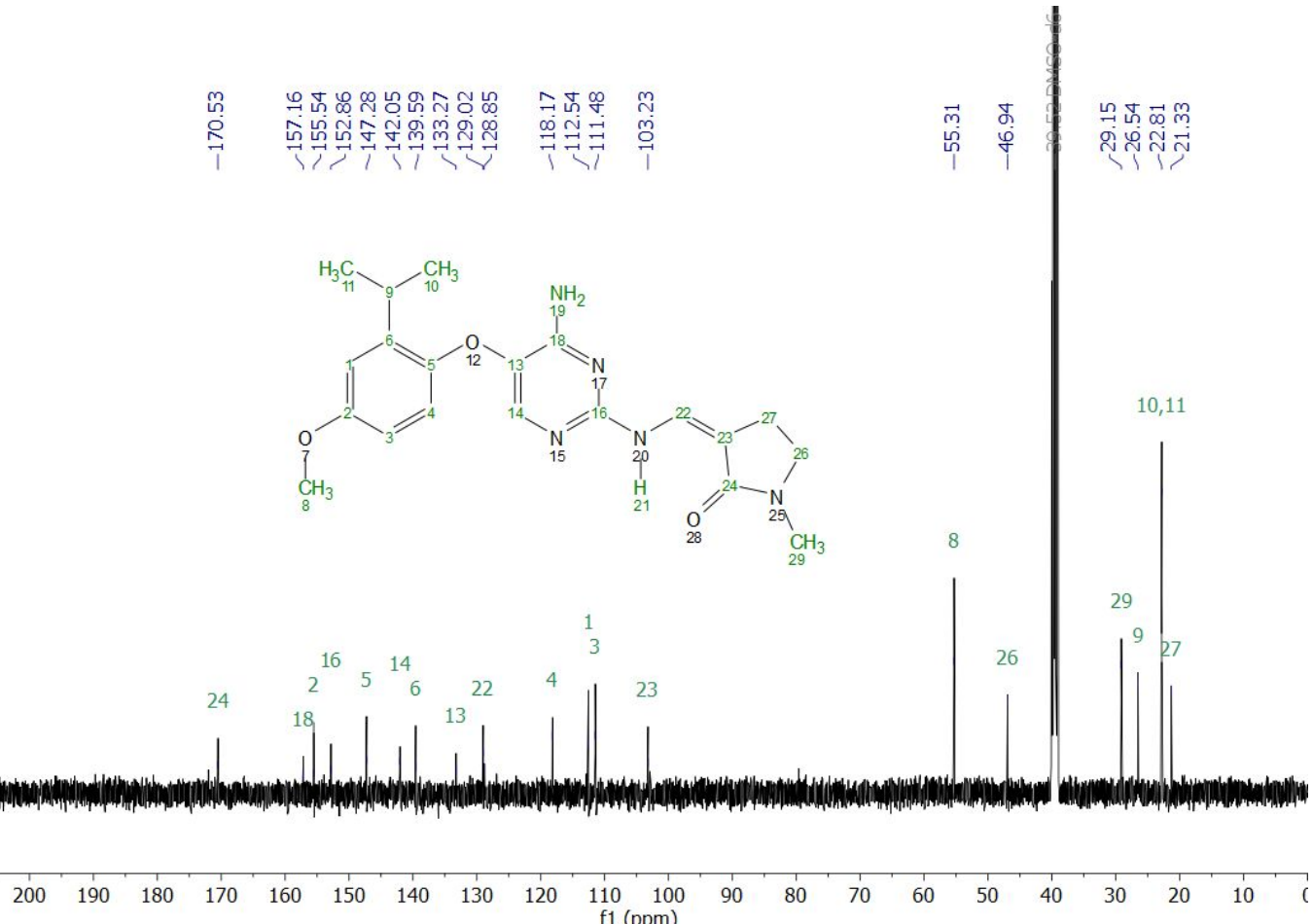

Figure S47. ${ }^{13} \mathrm{C}$ NMR $(126 \mathrm{MHz})$ spectrum of $\mathbf{1 4 b}$ in $d_{6}$-DMSO.

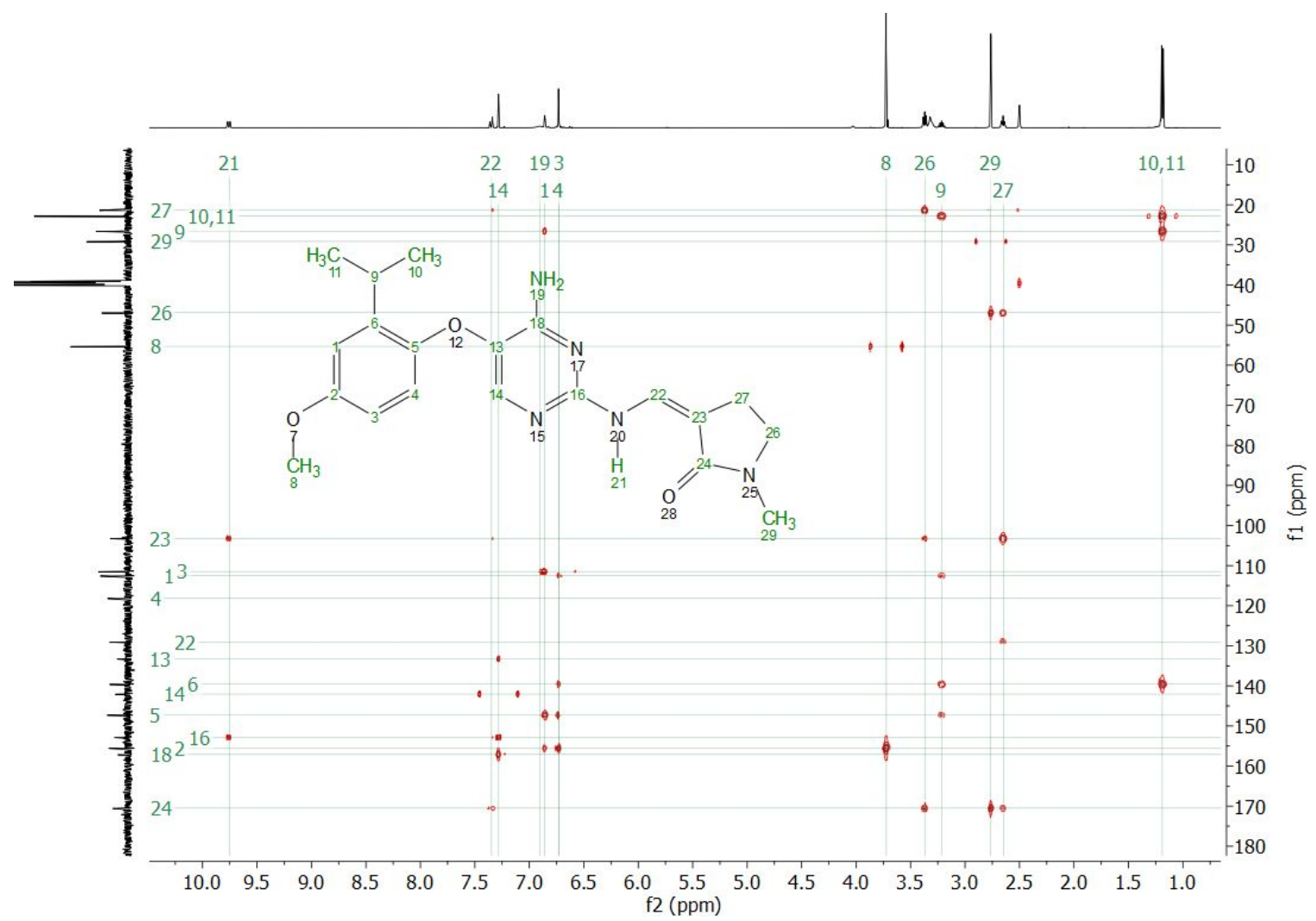

Figure S48. ${ }^{1} \mathrm{H}^{-13} \mathrm{C}$ HMBC NMR $(500 / 126 \mathrm{MHz})$ spectrum of $\mathbf{1 4 b}$ in $d_{6}$-DMSO. 


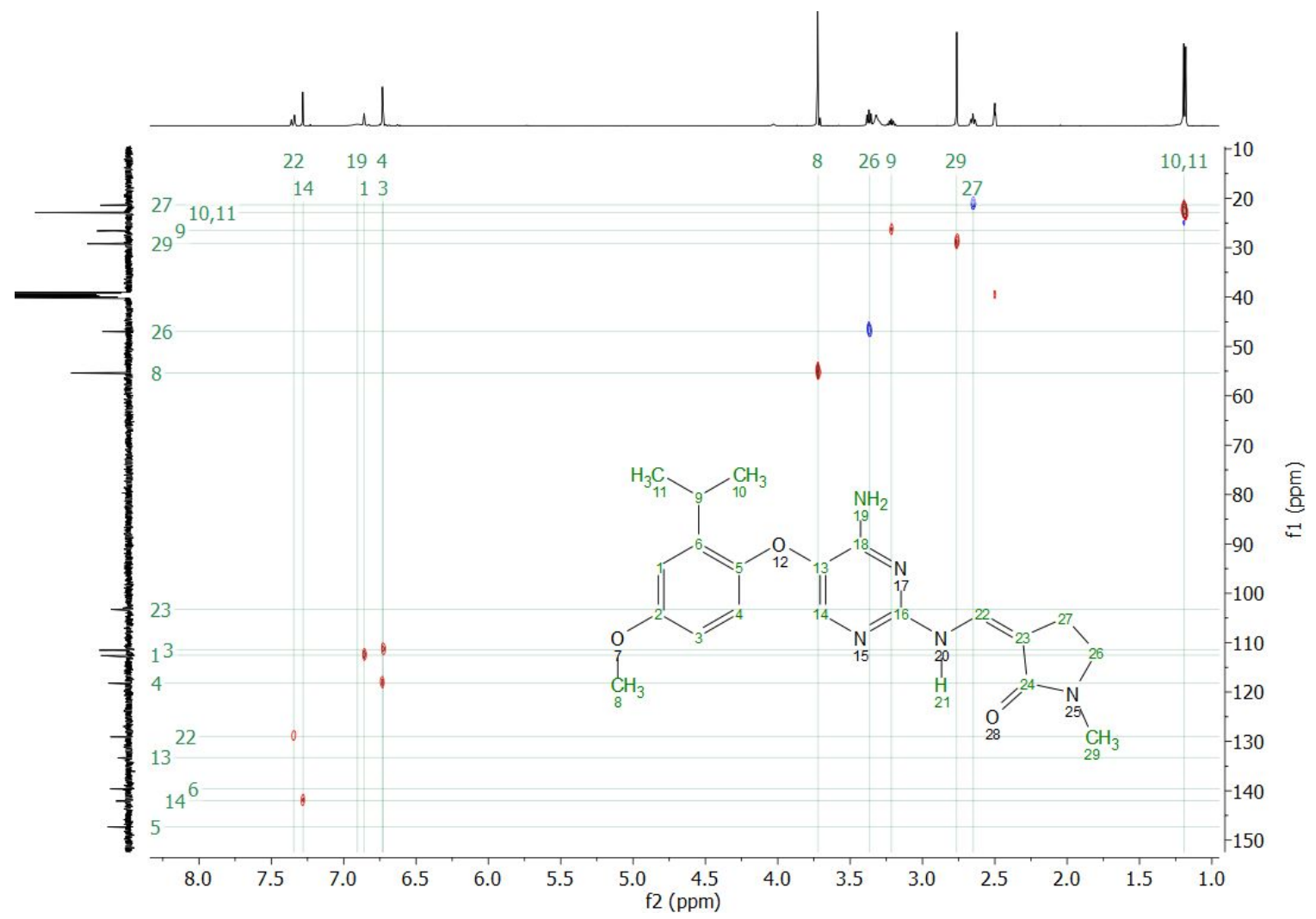

Figure S49. ${ }^{1} \mathrm{H}^{-13} \mathrm{C}$ HSQC NMR $(500 / 126 \mathrm{MHz})$ spectrum of $\mathbf{1 4 b}$ in $d_{6}$-DMSO.

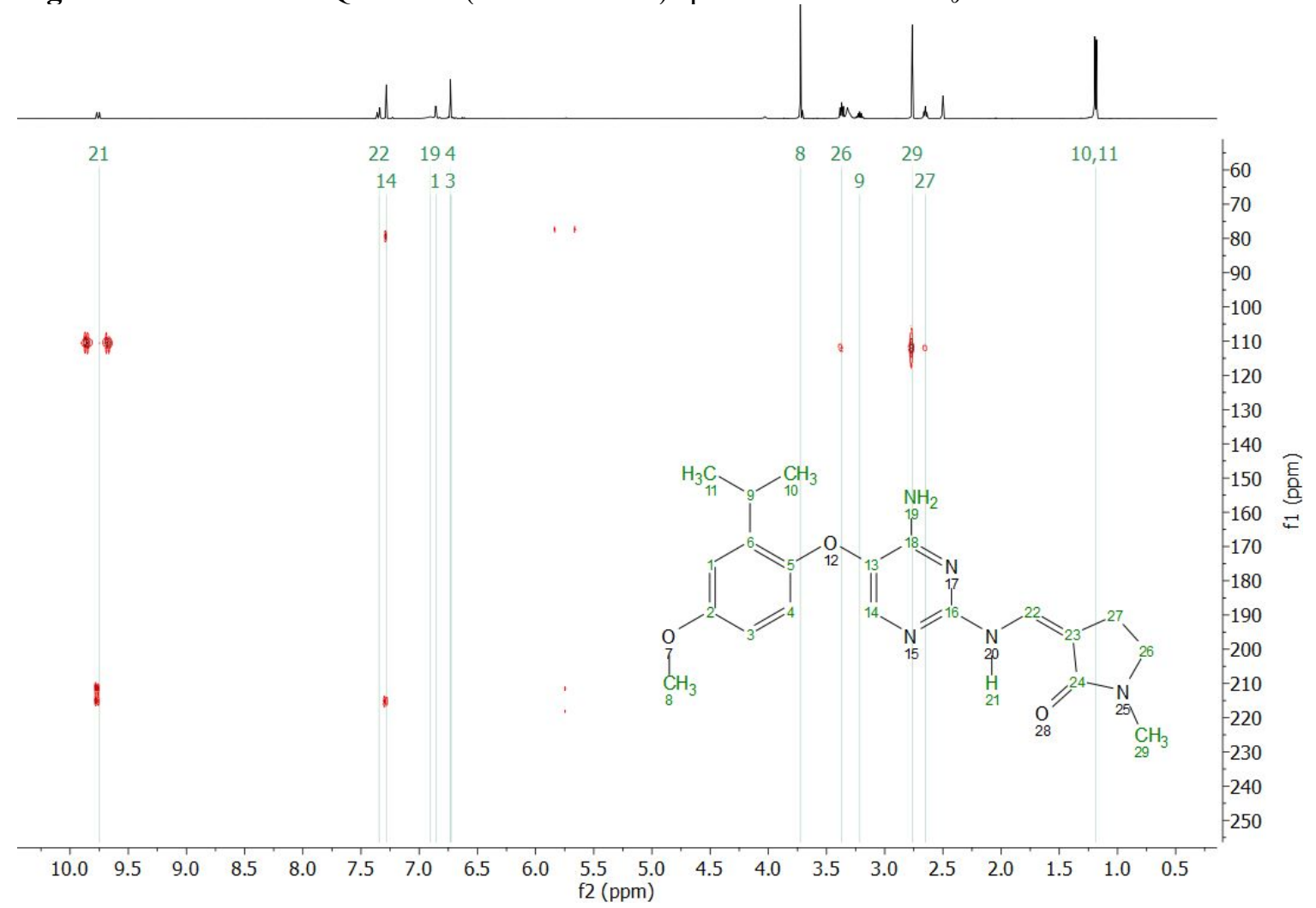

Figure S50. ${ }^{1} \mathrm{H}^{-15} \mathrm{~N}$ HMBC NMR (500/51 MHz) spectrum of 14b in $d_{\sigma}$-DMSO. 


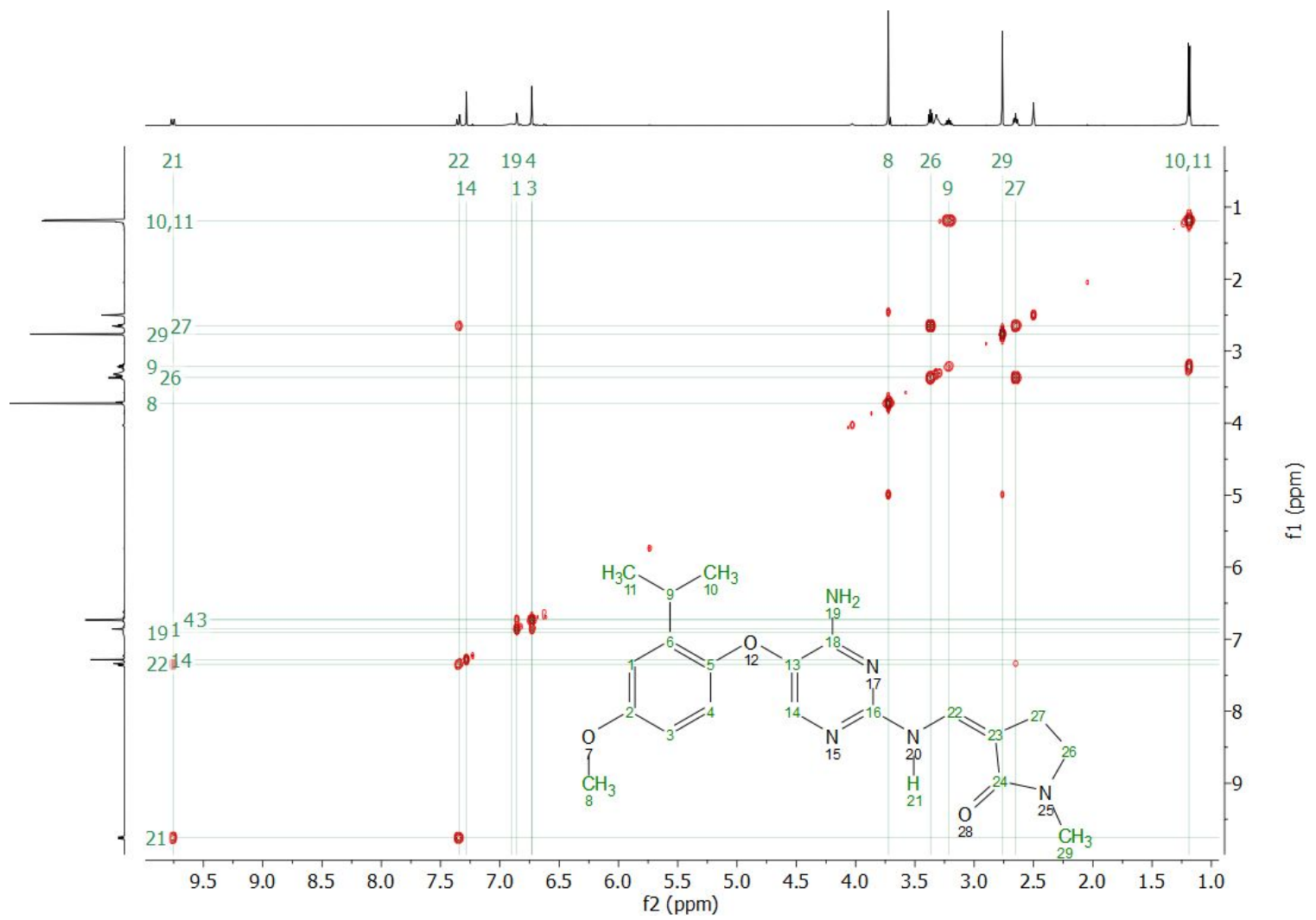

Figure S51. ${ }^{1} \mathrm{H}$ COSY NMR $(500 \mathrm{MHz})$ spectrum of $\mathbf{1 4 b}$ in $d_{\sigma}$-DMSO.
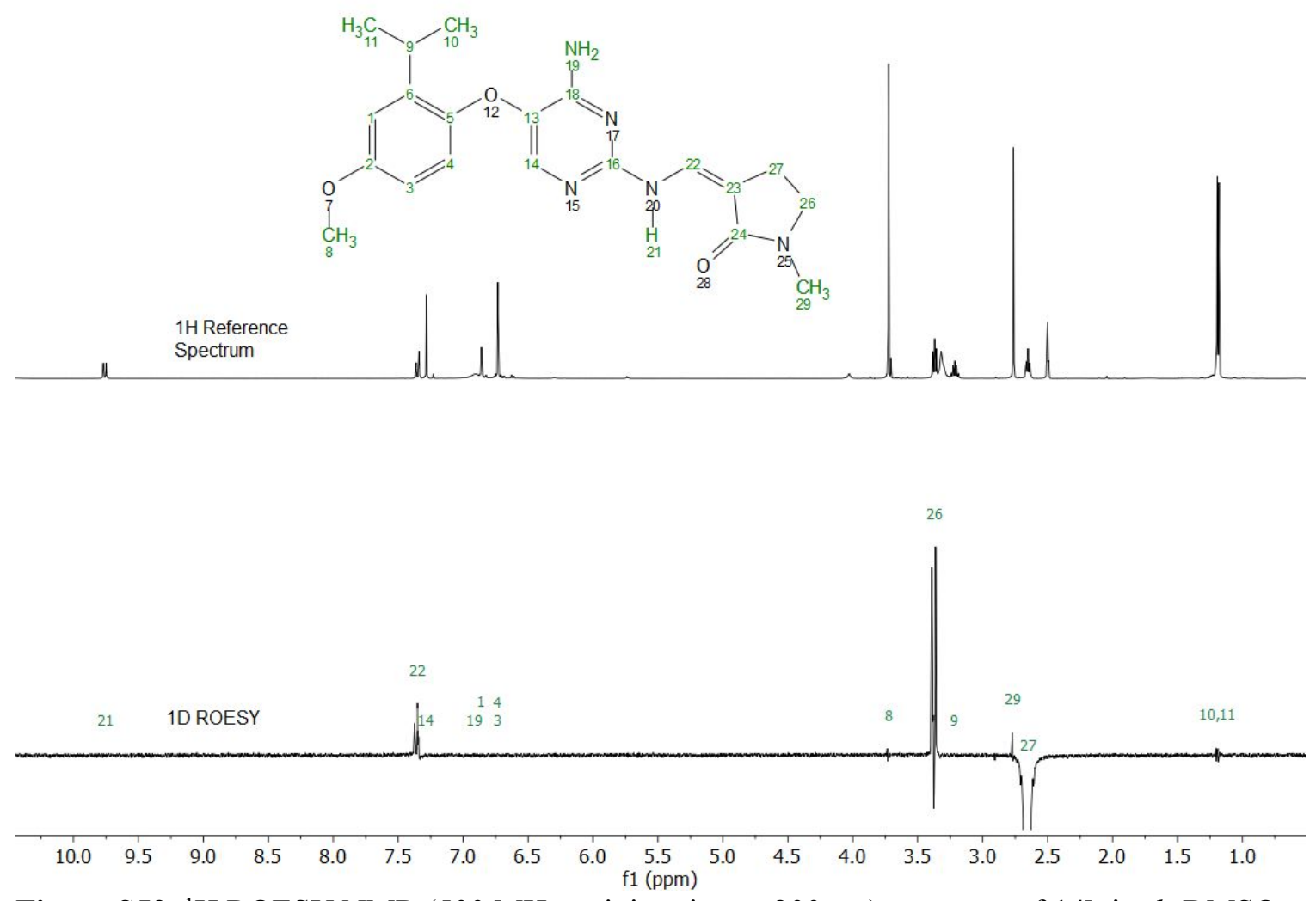

Figure S52. ${ }^{1} \mathrm{H}$ ROESY NMR $(500 \mathrm{MHz}$, mixing time $=200 \mathrm{~ms})$ spectrum of $\mathbf{1 4 b}$ in $d_{\sigma}$-DMSO. 


\section{DFT Computational Study}

\subsection{Computational Method for Modeling the Reactions in Scheme 2}

The quantum chemical calculations in this section were performed using the Gaussian $09^{1}$ or Gaussian $16^{2}$ program. Geometry optimizations and frequency calculations were conducted using the M06-2X functional ${ }^{3}$ and the $6-31 \mathrm{G}(\mathrm{d}, \mathrm{p})$ basis set. The stationary points were characterized as minima by the presence of only positive eigenvalues of the Hessian. Single-point (SP) energies were then calculated at the M06-2X/def2-TZVPP level. ${ }^{4}$ Default geometric and SCF convergence criteria were used. The Gibbs energies were calculated with the quasiharmonic approximation proposed by Cramer and Truhlar; ${ }^{5}$ this method is the same as the usual harmonic oscillator approximation, except that the vibrational frequencies lower than $100 \mathrm{~cm}^{-1}$ were raised to $100 \mathrm{~cm}^{-1}$ to correct for the breakdown of the harmonic oscillator approximation for low frequencies. 


\subsection{Cartesian Coordinates of DFT Optimized Structures in Scheme 2}

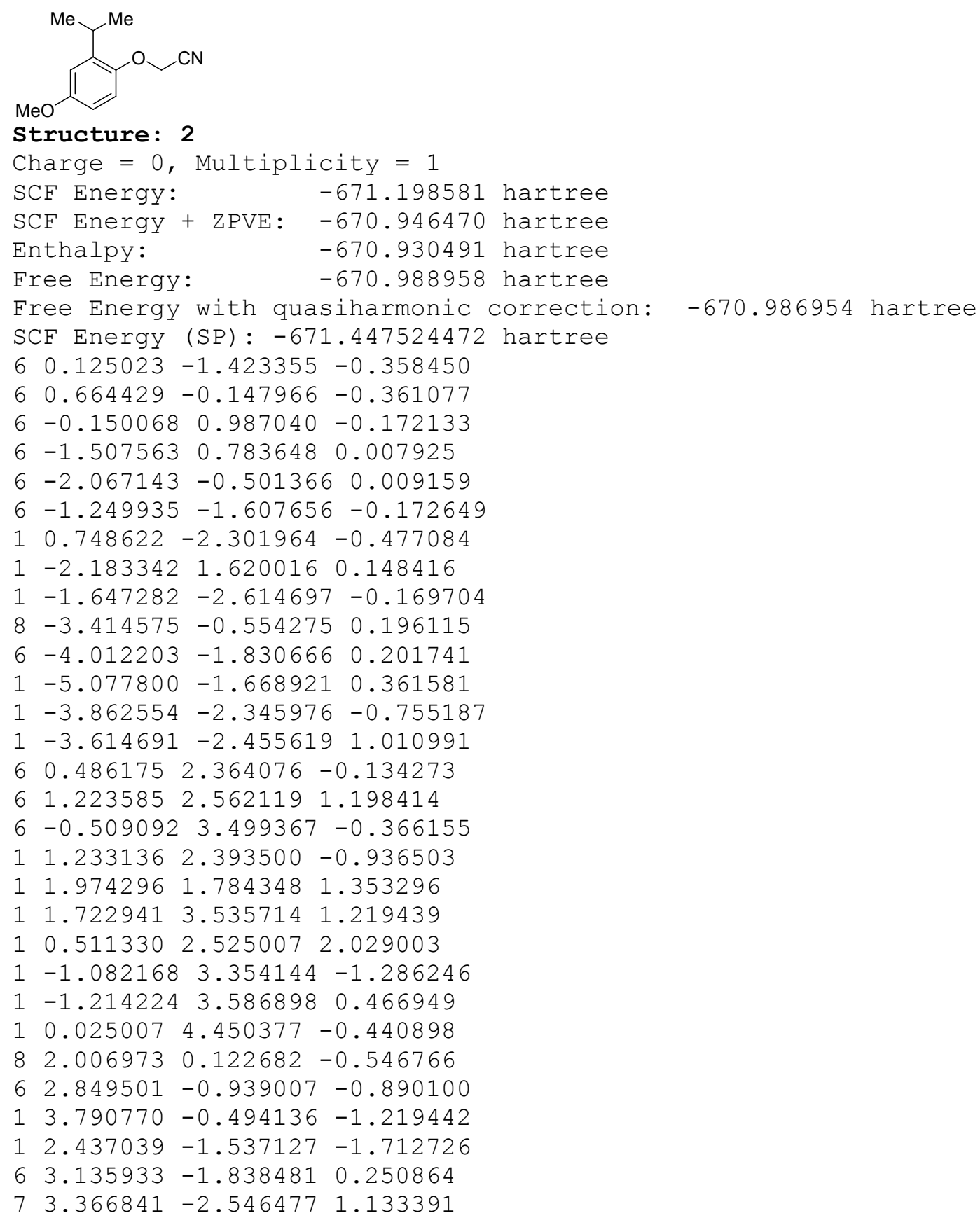




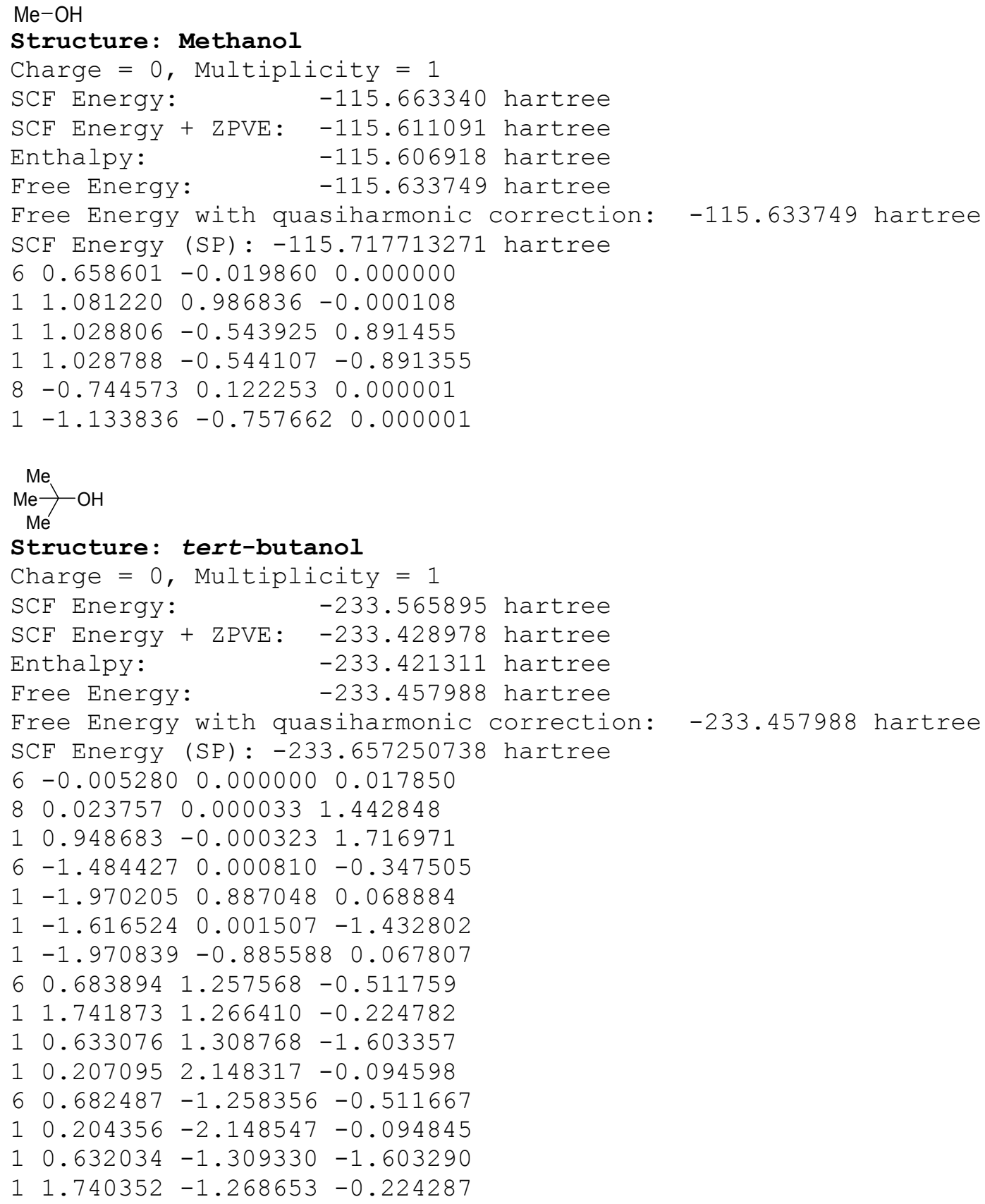




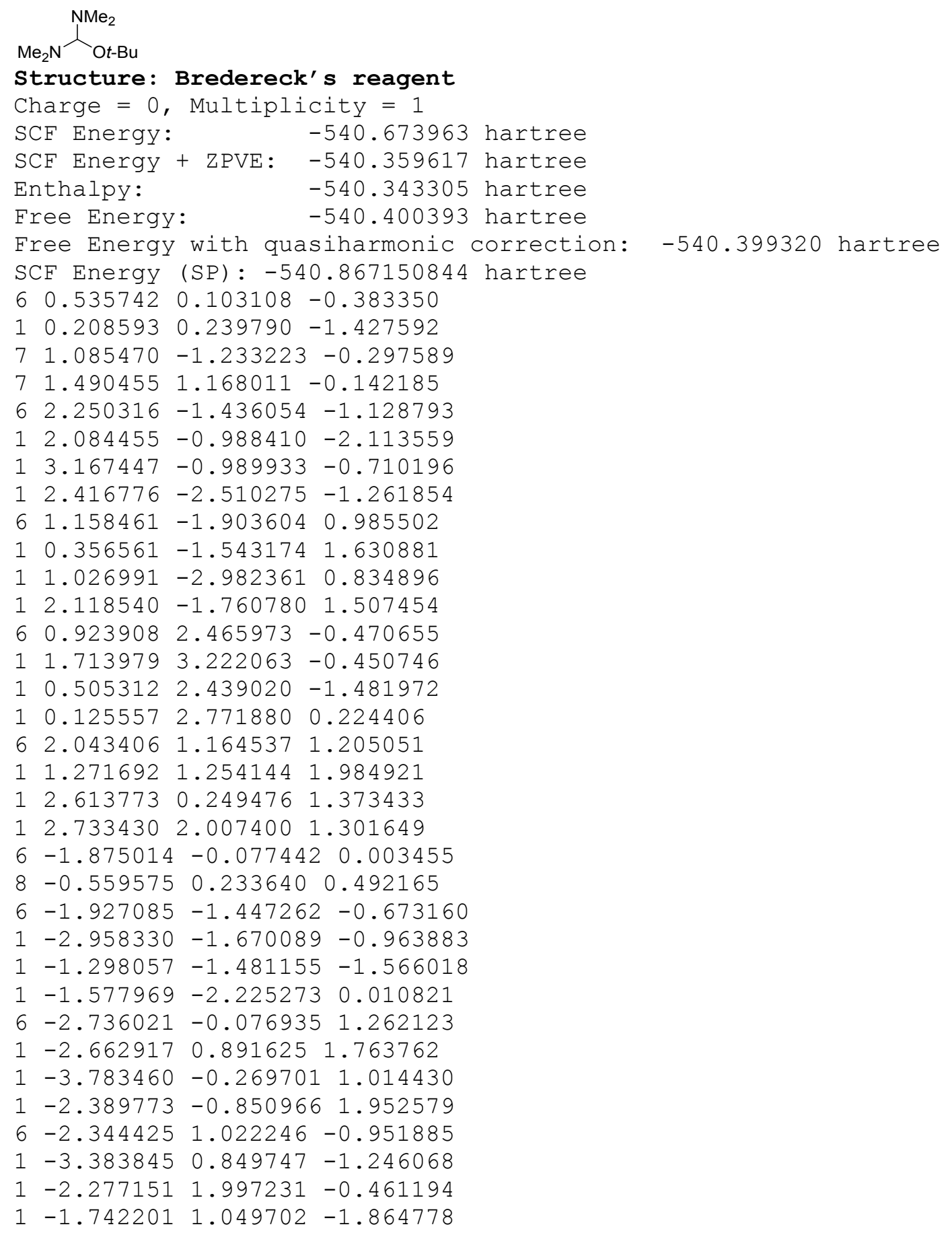




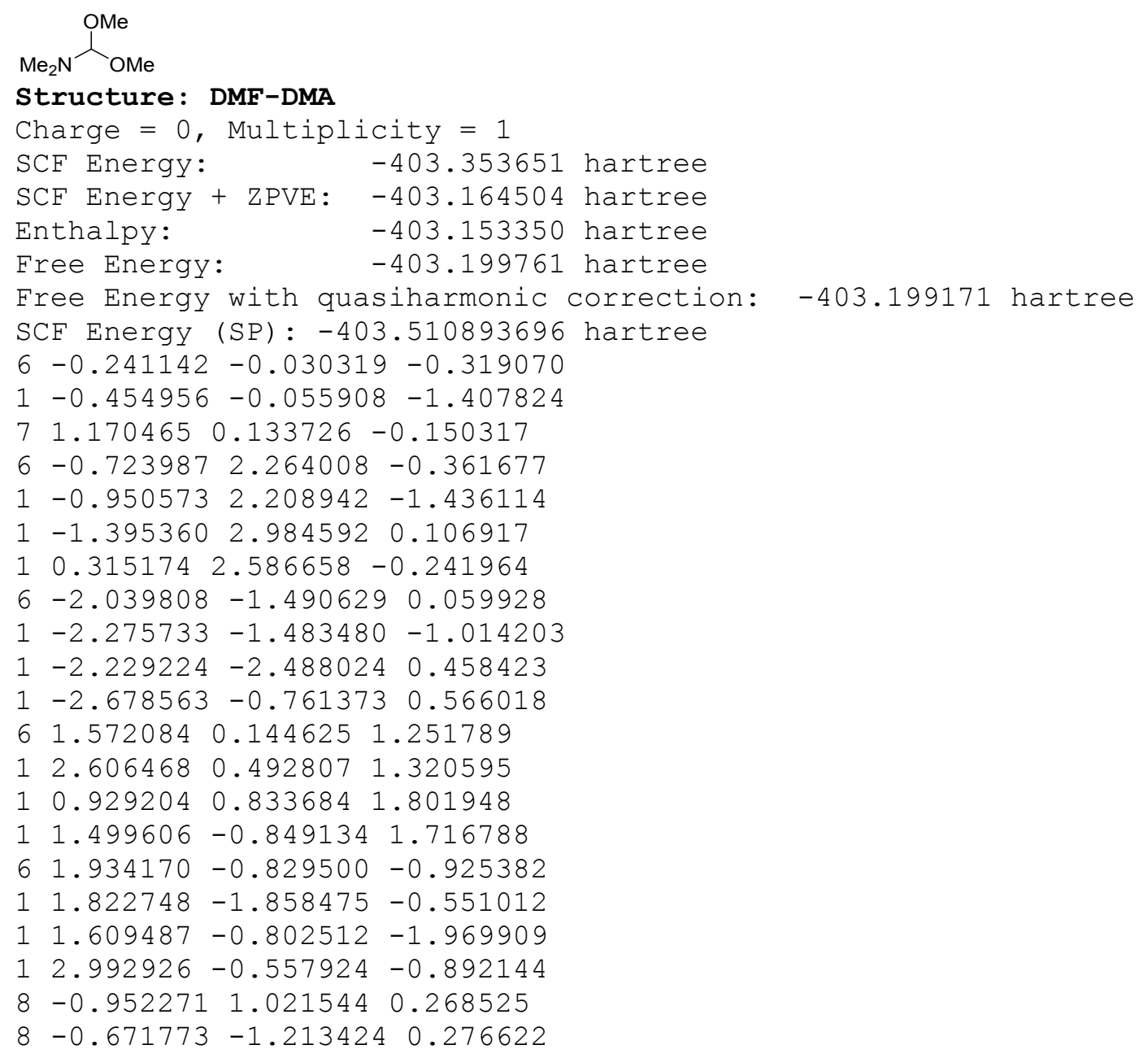




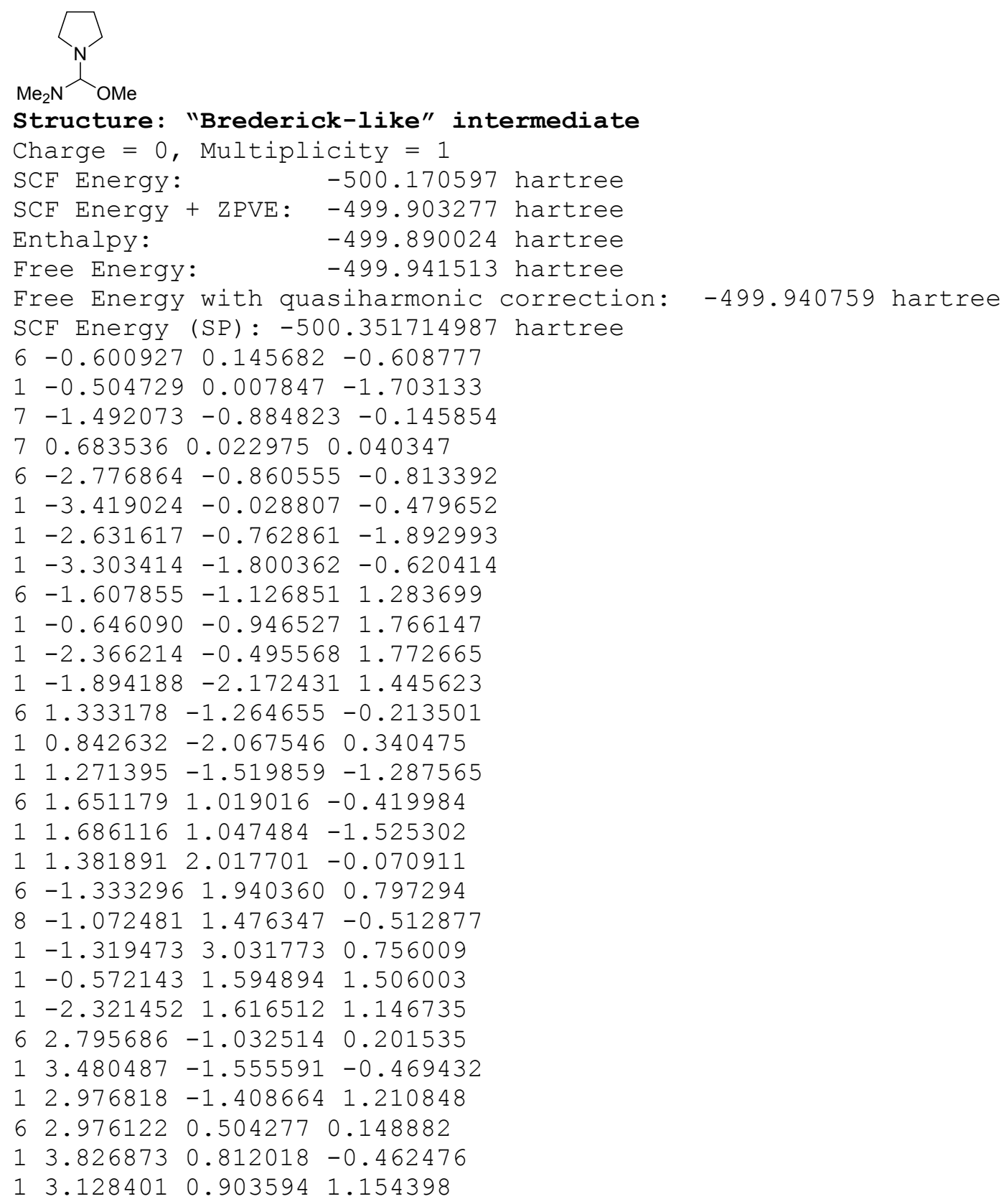




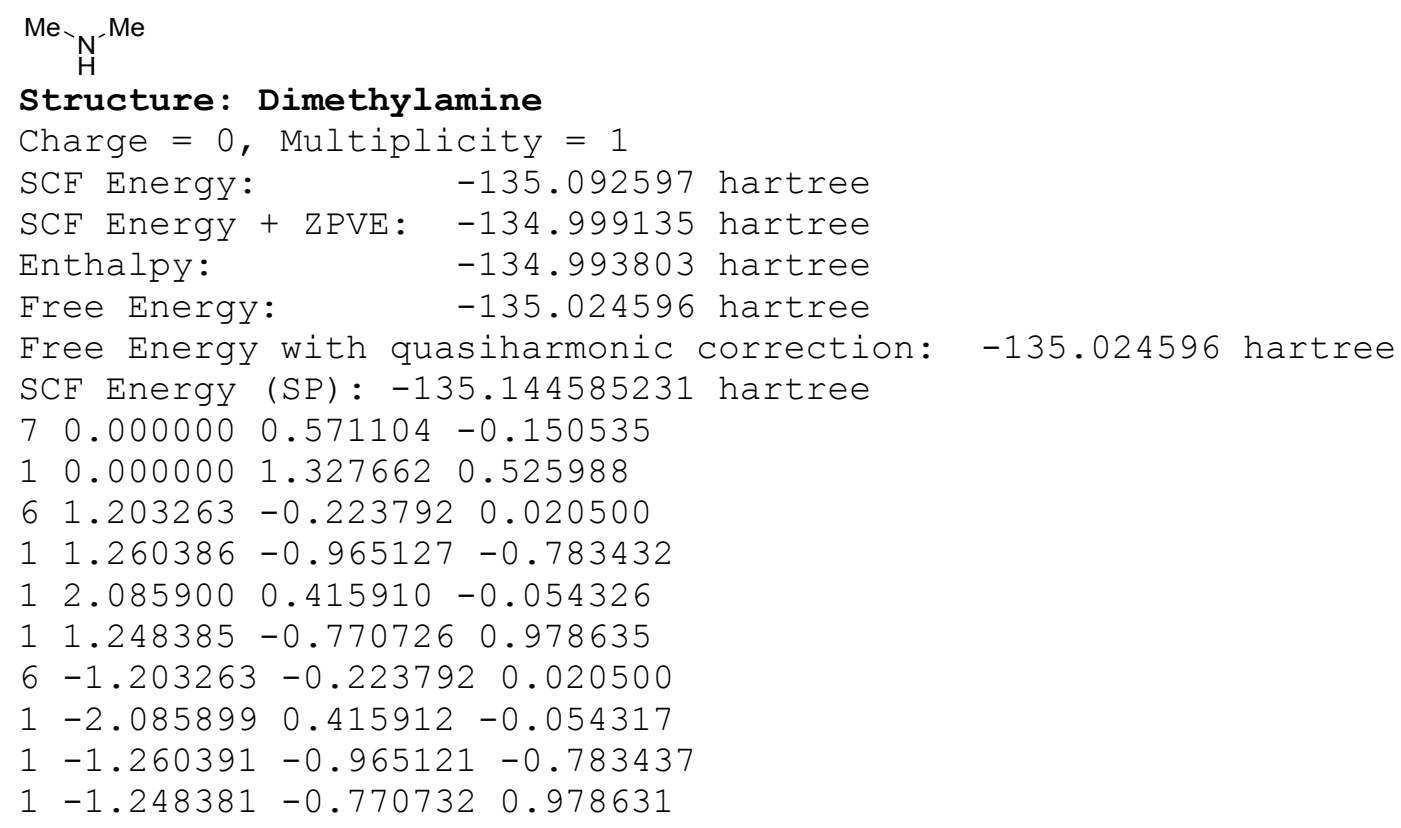




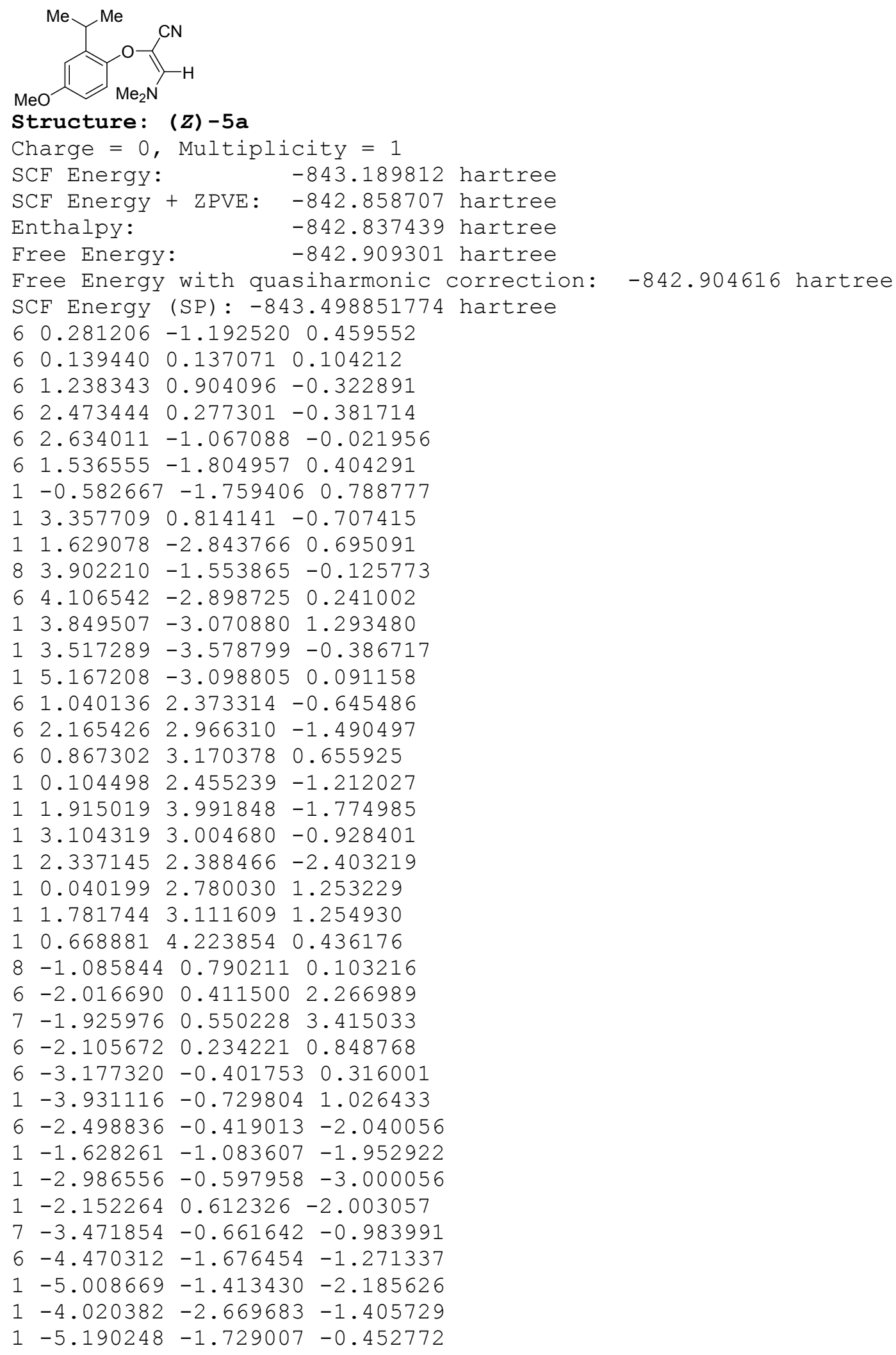




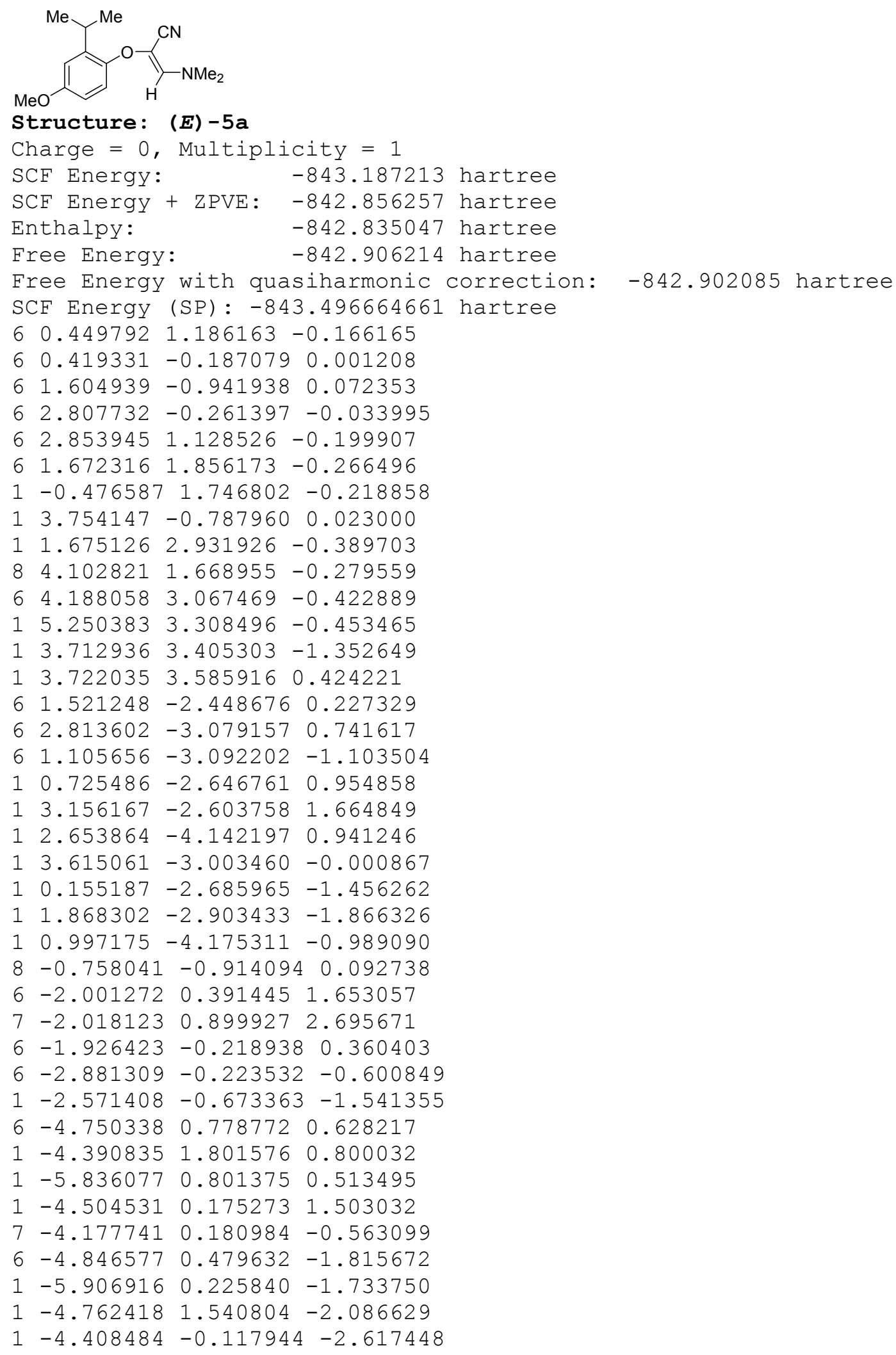




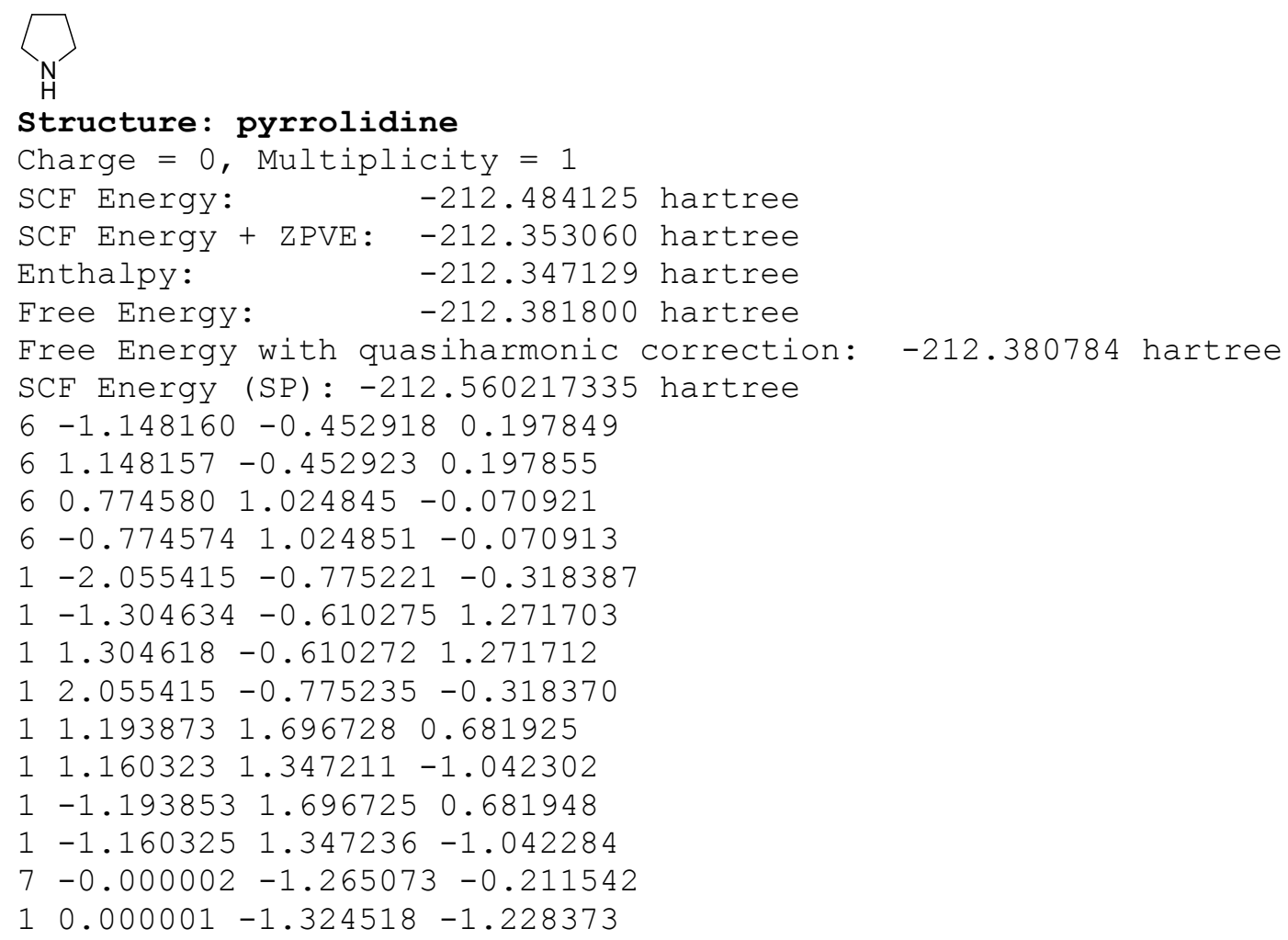


<smiles>COc1ccc(O/C=C\C(C#N)C(C)(C)C)c(C)c1</smiles>

\section{Structure: $(z)-5 b$}

Charge $=0$, Multiplicity $=1$

SCF Energy: $\quad-920.587434$ hartree

SCF Energy + ZPVE: -920.219052 hartree

Enthalpy: $\quad-920.197116$ hartree

Free Energy: $\quad-920.271752$ hartree

Free Energy with quasiharmonic correction: -920.265698 hartree

SCF Energy (SP) : -920.920776026 hartree

$6-0.838059-0.964449-1.000033$

$6-2.033095-1.645642-0.748969$

$6-2.965445-1.088712 \quad 0.117276$

$\begin{array}{llll}6 & -2.701190 & 0.145400 & 0.725205\end{array}$

$\begin{array}{lllll}6 & -1.526860 & 0.840187 & 0.481042\end{array}$

$\begin{array}{lllll}6 & -0.595251 & 0.258741 & -0.399864\end{array}$

$\begin{array}{llll}6 & -1.230526 & 2.200988 & 1.082803\end{array}$

$\begin{array}{llll}6 & -2.068251 & 2.514193 & 2.320700\end{array}$

$6-1.414263 \quad 3.2922090 .017560$

$6-4.468484-2.892803-0.160415$

$8-4.158989-1.6591790 .445196$

$\begin{array}{lllll}8 & 0.576908 & 0.975108 & -0.581215\end{array}$

$\begin{array}{lllll}6 & 1.471529 & 0.526598 & -1.533786\end{array}$

$\begin{array}{llll}6 & 2.629450 & -0.104028 & -1.211401\end{array}$

$\begin{array}{lllll}6 & 1.164041 & 0.816297 & -2.899034\end{array}$

$\begin{array}{llllll}7 & 0.905287 & 1.045817 & -4.006681\end{array}$

$\begin{array}{llll}6 & 2.319400 & -0.342523 & 1.259826\end{array}$

$\begin{array}{lllll}6 & 4.370195 & -1.118115 & 0.197990\end{array}$

$\begin{array}{llll}6 & 3.164519 & -1.141098 & 2.256930\end{array}$

$\begin{array}{lllll}6 & 4.587632 & -1.005181 & 1.705986\end{array}$

$73.069925-0.4752160 .005972$

$1-0.102889-1.395011-1.670408$

$\begin{array}{llll}1 & -2.207619 & -2.597443 & -1.234846\end{array}$

$\begin{array}{llll}1 & -3.453728 & 0.539042 & 1.399722\end{array}$

$\begin{array}{llll}1 & -0.172872 & 2.198178 & 1.374955\end{array}$

$\begin{array}{lllll}1 & -1.986794 & 1.727098 & 3.075876\end{array}$

$\begin{array}{lllll}1 & -1.733122 & 3.454685 & 2.766367\end{array}$

$\begin{array}{lllll}1 & -3.126126 & 2.633904 & 2.064527\end{array}$

$1-0.784702 \quad 3.100746-0.854026$

$\begin{array}{llll}1 & -2.458491 & 3.322443 & -0.309870\end{array}$

$\begin{array}{llll}1 & -1.154428 & 4.274072 & 0.425255\end{array}$

$\begin{array}{llll}1 & -3.736426 & -3.665441 & 0.106667\end{array}$

$\begin{array}{llll}1 & -5.449670 & -3.181361 & 0.215952\end{array}$

$1-4.510300-2.803179-1.252930$

$13.302006-0.332323-2.034367$

$12.240218 \quad 0.713505 \quad 1.542902$

$\begin{array}{lllll}1 & 1.306487 & -0.740929 & 1.151004\end{array}$

$14.336866-2.171172-0.115528$

$\begin{array}{llll}1 & 5.143227 & -0.609252 & -0.385471\end{array}$

$\begin{array}{llll}1 & 2.859994 & -2.192552 & 2.244386\end{array}$

$\begin{array}{lllll}1 & 3.059148 & -0.769544 & 3.277606\end{array}$ 
$14.992268-0.0171601 .947265$

$15.275066-1.7622912 .087155$<smiles>COc1ccc(OC(C#N)=C(C#N)N2CCCC2)c(C(C)C)c1</smiles>

Charge $=0$, Multiplicity $=1$

SCF Energy: $\quad-920.584280$ hartree

SCF Energy + ZPVE: -920.216472 hartree

Enthalpy: $\quad-920.195372$ hartree

Free Energy: $\quad-920.266470$ hartree

Free Energy with quasiharmonic correction: -920.262588 hartree SCF Energy (SP) : -920.917661034 hartree

$6-0.931275-1.144119-0.045336$

$6-2.085368-1.921219-0.180516$

$6-3.326013-1.297436-0.209228$

$\begin{array}{lllll}6 & -3.406388 & 0.096352 & -0.099682\end{array}$

$\begin{array}{lllll}6 & -2.273246 & 0.882378 & 0.039278\end{array}$

$\begin{array}{lllll}6 & -1.025199 & 0.232327 & 0.060074\end{array}$

$\begin{array}{lllll}1 & 0.039938 & -1.624828 & -0.022592\end{array}$

$\begin{array}{lllll}1 & -1.989443 & -2.997308 & -0.255278\end{array}$

$\begin{array}{lllll}1 & -4.395471 & 0.541010 & -0.116469\end{array}$

$\begin{array}{lllll}6 & -2.327262 & 2.395660 & 0.131983\end{array}$

$\begin{array}{llll}6 & -3.693395 & 2.929146 & 0.558454\end{array}$

$\begin{array}{llll}6 & -1.903379 & 3.020095 & -1.205227\end{array}$

$\begin{array}{lllll}1 & -1.589255 & 2.693125 & 0.886092\end{array}$

$1-4.040050 \quad 2.4596851 .483223$

$\begin{array}{lllll}1 & -3.635215 & 4.008697 & 0.722029\end{array}$

$\begin{array}{lllll}1 & -4.447599 & 2.757340 & -0.216771\end{array}$

$\begin{array}{lllll}1 & -1.899431 & 4.112341 & -1.135367\end{array}$

$1-0.903125 \quad 2.688903-1.492615$

$\begin{array}{lllll}1 & -2.605307 & 2.730064 & -1.994160\end{array}$

$6-4.478790-3.349064-0.442855$

$\begin{array}{llll}1 & -3.915057 & -3.667364 & -1.328787\end{array}$

$1-4.031397-3.8072550 .448116$

$1-5.513205-3.679193-0.536682$

$8-4.519014-1.945484-0.335409$

$\begin{array}{lllll}8 & 0.079450 & 1.062017 & 0.176758\end{array}$

$\begin{array}{lllll}6 & 1.297276 & 0.473182 & 0.479077\end{array}$

$\begin{array}{lllll}6 & 2.249170 & 0.463452 & -0.488591\end{array}$

$\begin{array}{lllll}1 & 1.929696 & 0.859555 & -1.450035\end{array}$

$\begin{array}{llll}6 & 1.433154 & -0.041692 & 1.805783\end{array}$

$\begin{array}{llll}7 & 1.511505 & -0.473688 & 2.880167\end{array}$

$\begin{array}{lllll}6 & 4.418459 & 0.101633 & -1.596298\end{array}$

$\begin{array}{lllll}6 & 4.206738 & -0.437254 & 0.768410\end{array}$

$65.583535-0.790573-1.171441$

$\begin{array}{lllll}1 & 4.758611 & 1.129842 & -1.784635\end{array}$

$\begin{array}{lllll}1 & 3.904814 & -0.260517 & -2.491816\end{array}$

$\begin{array}{lllll}6 & 5.673597 & -0.526367 & 0.335516\end{array}$

$13.804281-1.413857 \quad 1.065114$

$\begin{array}{lllll}1 & 4.050498 & 0.251917 & 1.603631\end{array}$

$15.331367-1.839559-1.355689$ 
$16.505173-0.557851-1.707645$

$16.218032-1.3006690 .878140$

$16.172566 \quad 0.431006 \quad 0.515855$

$7 \quad 3.5308390 .049923-0.434575$

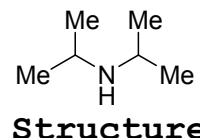

\section{Structure: diisopropylamine}

Charge $=0$, Multiplicity $=1$

SCF Energy: $\quad-292.284346$ hartree

SCF Energy + ZPVE: -292.076753 hartree

Enthalpy: $\quad-292.066556$ hartree

Free Energy: $\quad-292.109846$ hartree

Free Energy with quasiharmonic correction: -292.109685 hartree SCF Energy (SP) : -292.387246564 hartree

$\begin{array}{llll}6 & -1.223634 & 0.099860 & 0.257261\end{array}$

$\begin{array}{llll}6 & 1.219282 & 0.108209 & -0.258298\end{array}$

$7-0.015596-0.656099-0.073103$

$10.144501-1.3429290 .662885$

$\begin{array}{llll}6 & -2.365771-0.895032 & 0.434822\end{array}$

$1-2.525030-1.445551-0.496745$

$1-2.138326-1.619555 \quad 1.223852$

$1-3.292085-0.381601-0.706558$

$6-1.5464311 .091897-0.856215$

$\begin{array}{llll}1 & -1.589481 & 0.565221 & -1.814959\end{array}$

$\begin{array}{lllll}1 & -2.513791 & 1.567324 & -0.672356\end{array}$

$1-0.7960201 .883996-0.932751$

$\begin{array}{llll}6 & 1.557958 & 1.062613 & 0.891718\end{array}$

$11.647604 \quad 0.505876 \quad 1.832222$

$\begin{array}{lllll}1 & 2.509527 & 1.570347 & 0.707585\end{array}$

$\begin{array}{lllll}1 & 0.790633 & 1.830391 & 1.026001\end{array}$

$\begin{array}{llll}6 & 2.354082 & -0.883799 & -0.490741\end{array}$

$\begin{array}{lllll}1 & 3.284615 & -0.367068 & -0.739464\end{array}$

$\begin{array}{lllll}1 & 2.533560 & -1.475682 & 0.415175\end{array}$

$12.098437-1.568591-1.302175$

$\begin{array}{llll}1 & -1.115301 & 0.667713 & 1.199294\end{array}$

$\begin{array}{llll}1 & 1.097413 & 0.700317 & -1.174683\end{array}$ 


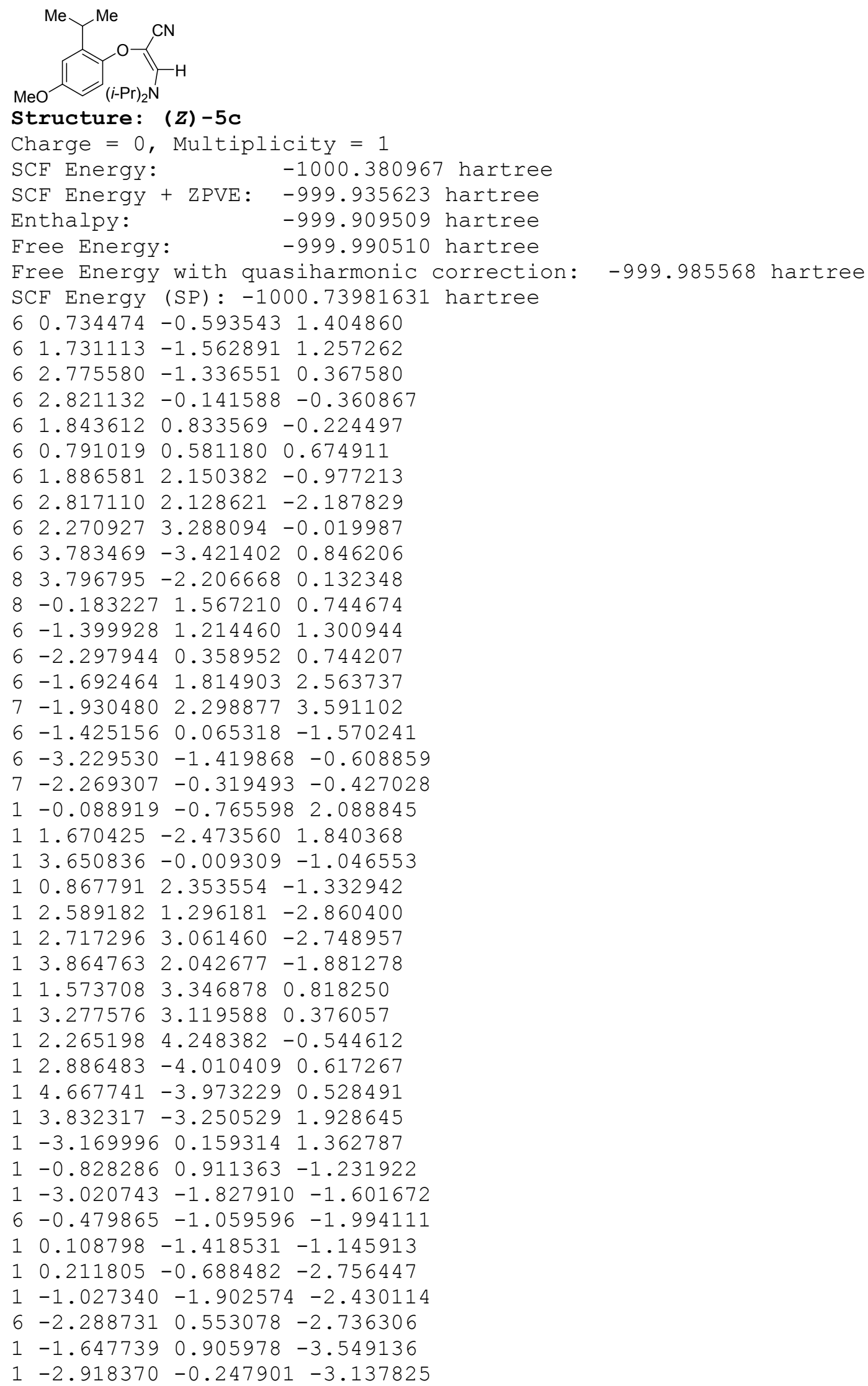


$\begin{array}{llll}1 & -2.933083 & 1.377902 & -2.421013 \\ 6 & -4.681399 & -0.938262 & -0.600096 \\ 1 & -5.353231 & -1.770136 & -0.828555 \\ 1 & -4.969782 & -0.544769 & 0.379507 \\ 1 & -4.833871 & -0.152075 & -1.343511 \\ 6 & -2.982907 & -2.531202 & 0.411300 \\ 1 & -3.627412 & -3.390423 & 0.206452 \\ 1 & -1.938925 & -2.854079 & 0.375707 \\ 1 & -3.198822 & -2.185706 & 1.427761\end{array}$

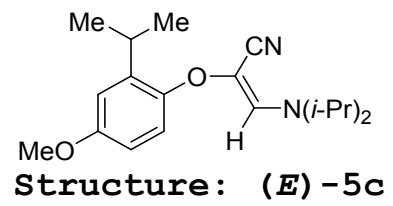

Charge $=0$, Multiplicity $=1$

SCF Energy: $\quad-1000.375590$ hartree

SCF Energy + ZPVE: -999.930850 hartree

Enthalpy: $\quad-999.904257$ hartree

Free Energy: $\quad-999.987758$ hartree

Free Energy with quasiharmonic correction: -999.981428 hartree

SCF Energy (SP) : -1000.73433338 hartree

$\begin{array}{llll}6 & 3.481099 & 2.230367-1.960995\end{array}$

$62.5139321 .983092-0.797366$

$\begin{array}{llll}6 & 2.913853 \quad 2.799253 \quad 0.436093\end{array}$

$6 \quad 2.424699 \quad 0.502496-0.479891$

$61.258293-0.223316-0.699700$

$\begin{array}{lllll}8 & 0.155352 & 0.409094 & -1.270308\end{array}$

$\begin{array}{lllll}6 & -0.924701 & 0.564547 & -0.405591\end{array}$

$\begin{array}{llll}6 & -0.664622 & 1.406474 & 0.720074\end{array}$

$\begin{array}{llll}7 & -0.390023 & 2.074133 & 1.629723\end{array}$

$6-2.078442-0.070883-0.728901$

$\begin{array}{lllll}7 & -3.327715 & -0.020379 & -0.198439\end{array}$

$\begin{array}{llll}6 & -3.631860 & 0.858992 & 0.936880\end{array}$

$6-4.228975-1.147686-0.498000$

$\begin{array}{lllll}6 & 1.177339 & -1.580257 & -0.385964\end{array}$

$\begin{array}{lllll}6 & 2.273777 & -2.240517 & 0.134293\end{array}$

$\begin{array}{llll}6 & 3.465336 & -1.535793 & 0.345677\end{array}$

$\begin{array}{lllll}8 & 4.496081 & -2.264224 & 0.855297\end{array}$

$\begin{array}{llll}6 & 5.709423 & -1.589386 & 1.097633\end{array}$

$\begin{array}{lllll}6 & 3.534562 & -0.180135 & 0.039790\end{array}$

$\begin{array}{llll}1 & 3.511707 & 3.294228 & -2.214615\end{array}$

$14.497360 \quad 1.919681-1.694869$

$\begin{array}{lllll}1 & 3.179498 & 1.670762 & -2.850197\end{array}$

$\begin{array}{lllll}1 & 1.516684 & 2.306633 & -1.109164\end{array}$

$\begin{array}{llll}1 & 2.212056 & 2.635788 & 1.257361\end{array}$

$\begin{array}{lllll}1 & 3.919064 & 2.531928 & 0.779595\end{array}$

$12.920505 \quad 3.866356 \quad 0.194485$

$\begin{array}{lllll}1 & -1.979194 & -0.714297 & -1.600505\end{array}$

$\begin{array}{lllll}1 & -2.959740 & 1.713406 & 0.831847\end{array}$

$\begin{array}{llll}6 & -5.054481 & 1.413491 & 0.860015\end{array}$

$6-4.712371-1.096243-1.946749$

$6-3.604542-2.501734-0.151646$

$\begin{array}{lllll}1 & 0.241022 & -2.103419 & -0.556698\end{array}$ 
$12.241865-3.2950330 .383700$

$\begin{array}{llll}1 & 6.396339 & -2.332479 & 1.501564\end{array}$

$16.127842-1.1730950 .173126$

$\begin{array}{lllll}1 & 5.579016 & -0.780547 & 1.826705\end{array}$

$14.450245 \quad 0.375692 \quad 0.208408$

$\begin{array}{llll}6 & -3.353327 & 0.178343 & 2.277377\end{array}$

$\begin{array}{llll}1 & -5.096924 & -1.008998 & 0.150957\end{array}$

$\begin{array}{lllll}1 & -5.812599 & 0.663460 & 1.102836\end{array}$

$\begin{array}{lllll}1 & -5.159207 & 2.222869 & 1.586817\end{array}$

$1-5.2617301 .815361-0.134934$

$1-3.4820150 .887909 \quad 3.099155$

$1-4.045938-0.6562292 .435596$

$1-2.329079-0.201265 \quad 2.315415$

$1-5.428192-1.900478-2.137001$

$\begin{array}{llll}1 & -5.193657 & -0.138692 & -2.157566\end{array}$

$1-3.880215-1.221715-2.646640$

$\begin{array}{lllll}1 & -3.233461 & -2.507810 & 0.876438\end{array}$

$1-4.344956-3.298815-0.260746$

$1-2.767541-2.733062-0.818132$

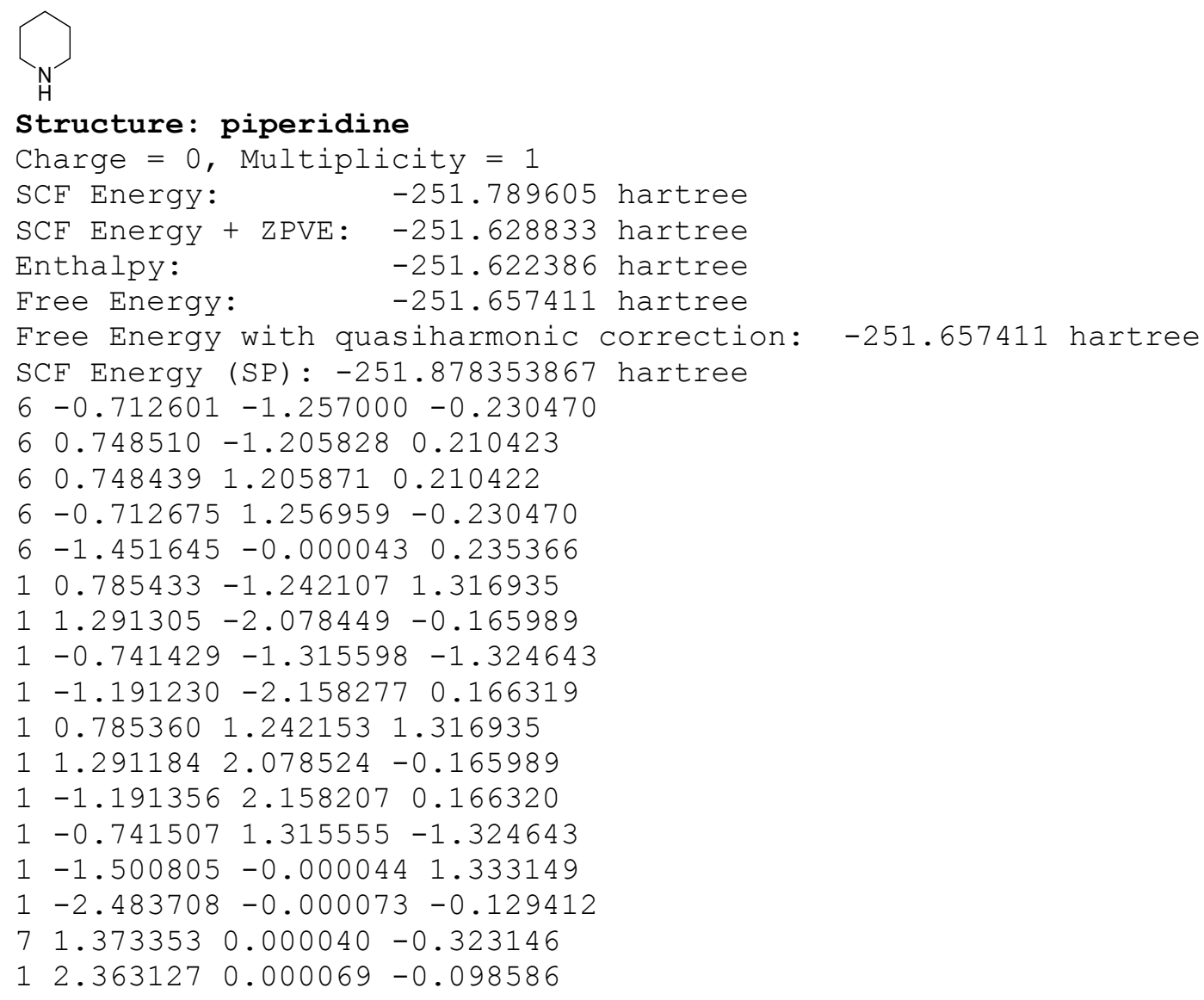




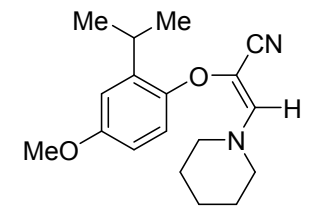

\section{Structure: $(z)-5 d$}

Charge $=0$, Multiplicity $=1$

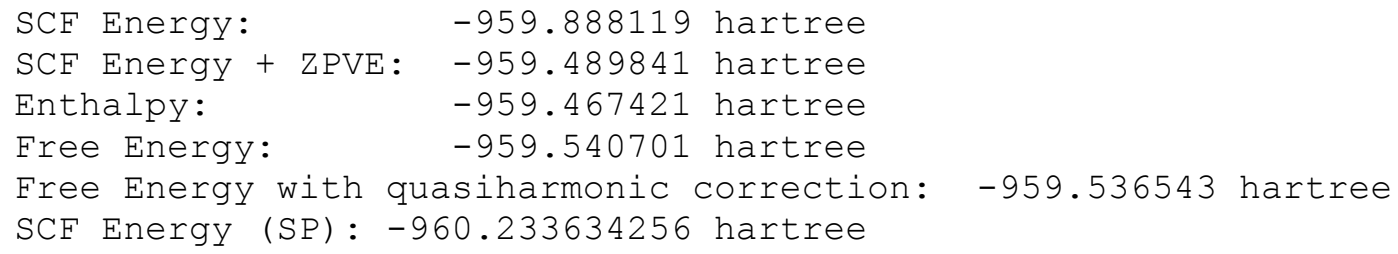


$\begin{array}{llll}1 & -3.214873 & 3.780411 & -0.245911 \\ 1 & -1.996140 & 2.869600 & 0.662056 \\ 1 & -0.962718 & 3.847142 & -1.385362 \\ 1 & -2.187047 & 3.073645 & -2.388444 \\ 7 & -2.573689 & 0.454843 & -0.424717\end{array}$

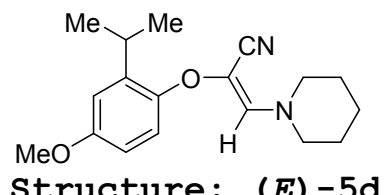

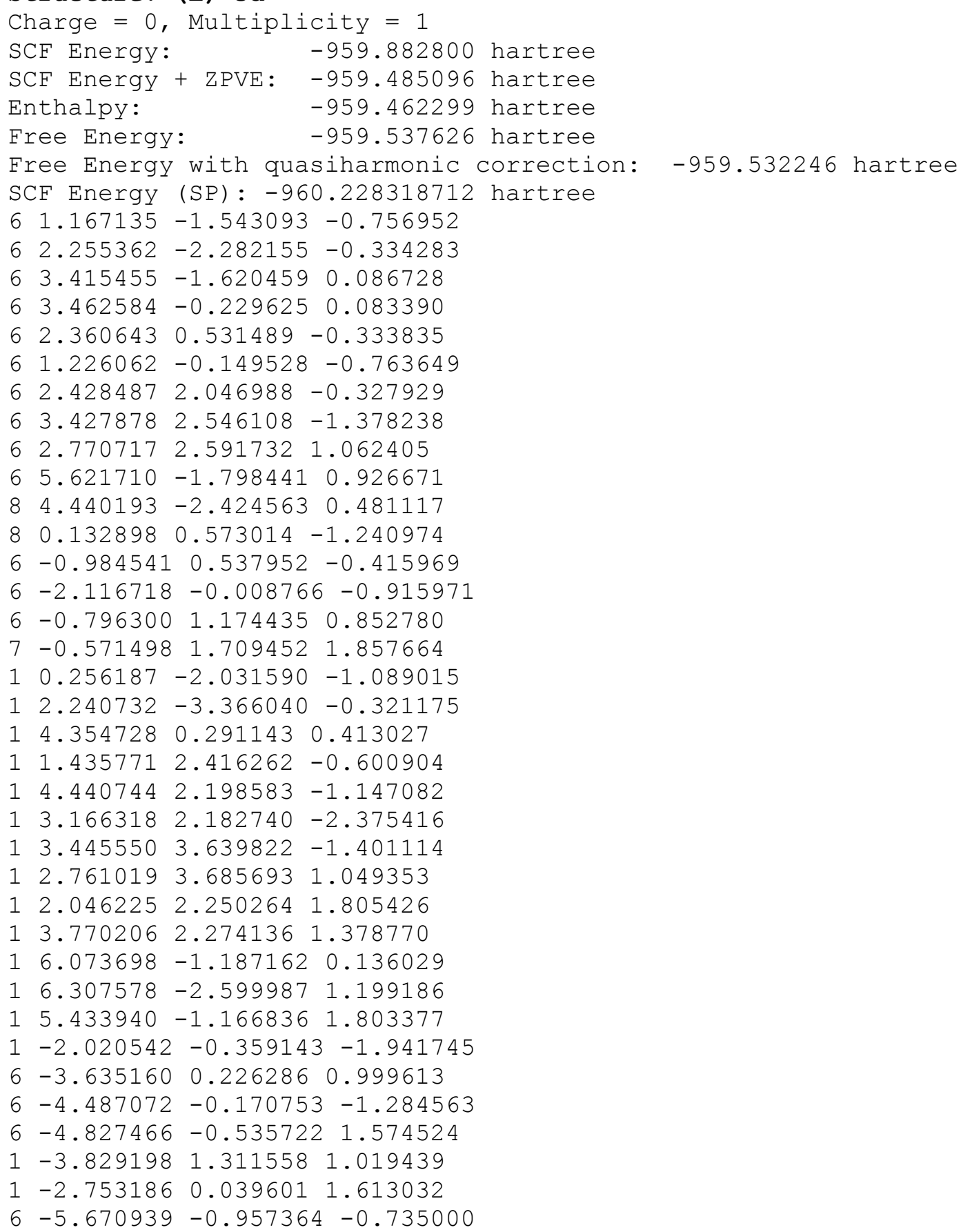


$\begin{array}{llll}1 & -4.791074 & 0.875047 & -1.458605 \\ 1 & -4.159885 & -0.580941 & -2.244301 \\ 6 & -6.044314 & -0.447647 & 0.656098 \\ 1 & -5.052656 & -0.134260 & 2.566797 \\ 1 & -4.542892 & -1.586661 & 1.701405 \\ 1 & -6.512457 & -0.865993 & -1.428414 \\ 1 & -5.399028 & -2.017748 & -0.678665 \\ 1 & -6.879623 & -1.021717 & 1.067097 \\ 1 & -6.374999 & 0.597206 & 0.583869 \\ 7 & -3.348933 & -0.209481 & -0.364298\end{array}$

$\widehat{\substack{\mathrm{N} \\ \mathrm{H}}}$

Structure: azetidine

Charge $=0$, Multiplicity $=1$

SCF Energy: $\quad-173.157401$ hartree

SCF Energy + ZPVE: -173.056320 hartree

Enthalpy: $\quad-173.051440$ hartree

Free Energy: $\quad-173.082249$ hartree

Free Energy with quasiharmonic correction: -173.082249 hartree SCF Energy (SP) : -173.221209287 hartree

$6-1.043827 \quad 0.041079 \quad 0.103722$

$60.000139-1.076543-0.086671$

$61.043813 \quad 0.041348 \quad 0.103722$

$\begin{array}{llll}1 & -1.921172 & 0.045873 & -0.549659\end{array}$

$\begin{array}{lllll}1 & -1.377586 & 0.098821 & 1.150138\end{array}$

$10.000204-1.462167-1.107166$

$\begin{array}{llll}1 & 0.000251 & -1.902258 & 0.624765\end{array}$

$\begin{array}{lllll}1 & 1.921164 & 0.046367 & -0.549649\end{array}$

$\begin{array}{llll}1 & 1.377556 & 0.099147 & 1.150141\end{array}$

$7-0.0001321 .023362-0.249501$

$\begin{array}{llll}1 & -0.000244 & 1.875380 & 0.303297\end{array}$<smiles>COc1ccc(O/C(C#N)=C\N2CCC2)c(C(C)C)c1</smiles>

\section{Structure: $(z)-5 e$}

Charge $=0$, Multiplicity $=1$

SCF Energy: $\quad-881.259679$ hartree

SCF Energy + ZPVE: -880.921690 hartree

Enthalpy: $\quad-880.900674$ hartree

Free Energy: $\quad-880.971923$ hartree

Free Energy with quasiharmonic correction: -880.967699 hartree SCF Energy (SP) : -881.580424064 hartree

$6-0.5224291 .131014-0.712968$

$6-1.7685311 .730982-0.505141$

$6-2.770508 \quad 1.0114340 .133806$

$6-2.525175-0.301648 \quad 0.555990$

$6-1.300474-0.9167120 .348584$

$6-0.298029-0.169478-0.296618$

$6-1.019661-2.3550680 .740979$

$6-1.027021-3.245425-0.510707$ 


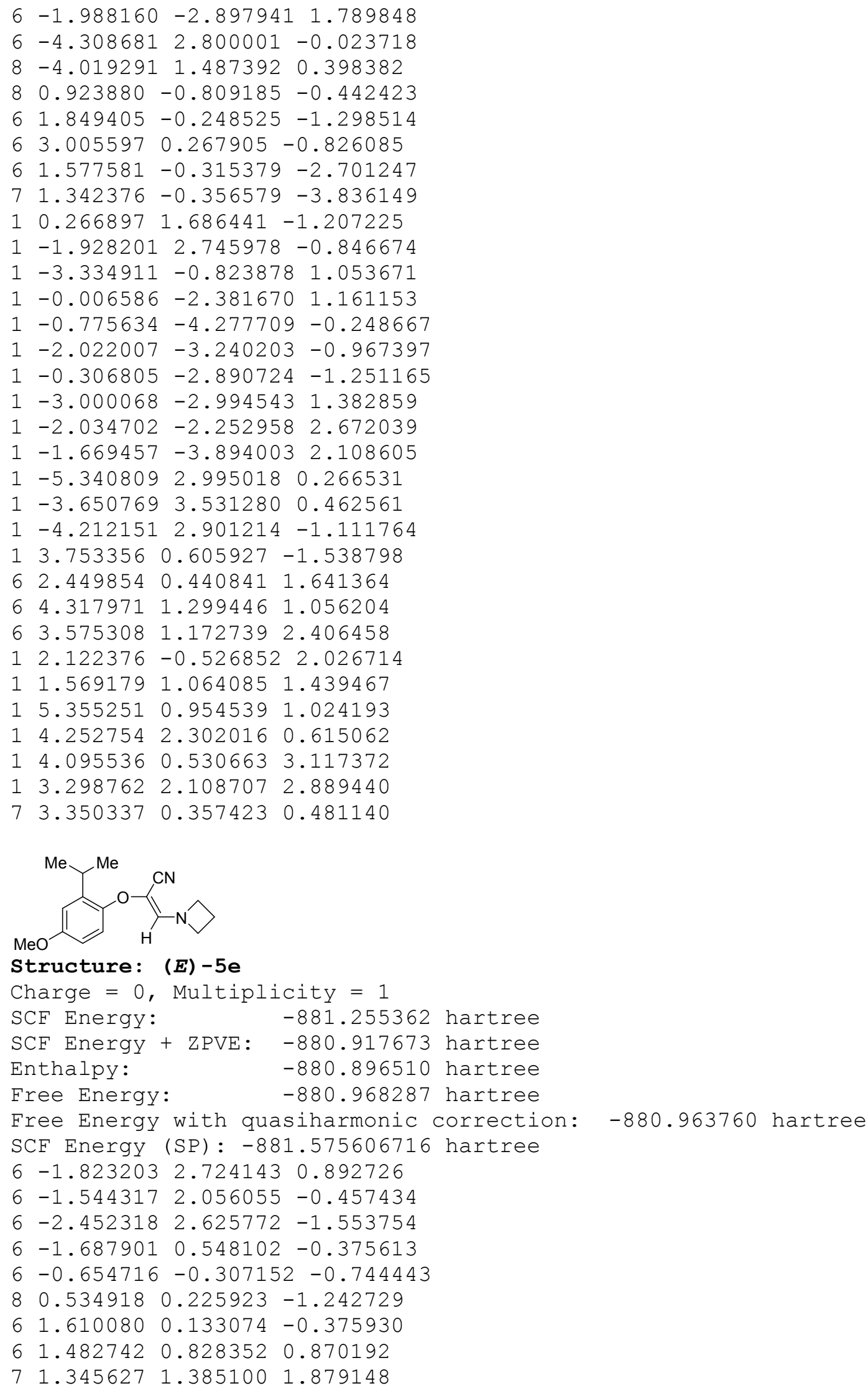




$\begin{array}{llll}6 & 2.703253 & -0.570623 & -0.741985 \\ 7 & 3.860681 & -0.721447 & -0.045123 \\ 6 & 5.173226 & -1.010570 & -0.636927 \\ 6 & 5.828557 & -0.329946 & 0.586573 \\ 6 & 4.424262 & 0.194381 & 0.958620 \\ 6 & -0.788340 & -1.692466 & -0.656083 \\ 6 & -1.972756 & -2.248342 & -0.212211 \\ 6 & -3.034414 & -1.409346 & 0.148430 \\ 8 & -4.164548 & -2.039925 & 0.568793 \\ 6 & -5.253056 & -1.232284 & 0.955551 \\ 6 & -2.888689 & -0.027818 & 0.064807 \\ 1 & -1.165433 & 2.327280 & 1.669573 \\ 1 & -1.658040 & 3.803143 & 0.818744 \\ 1 & -2.861738 & 2.569940 & 1.204783 \\ 1 & -0.506874 & 2.268019 & -0.732381 \\ 1 & -2.317677 & 3.708199 & -1.638972 \\ 1 & -2.232277 & 2.171594 & -2.523328 \\ 1 & -3.506118 & 2.435466 & -1.322892 \\ 1 & 2.657038 & -1.088124 & -1.697678 \\ 1 & 5.376068 & -2.075177 & -0.782687 \\ 1 & 5.344993 & -0.473299 & -1.577971 \\ 1 & 6.581460 & 0.425555 & 0.368021 \\ 1 & 4.257582 & 1.246197 & 0.693479 \\ 1 & 4.072418 & 0.024855 & 1.978181 \\ 1 & 0.050389 & -2.319674 & -0.941972 \\ 1 & -2.108317 & -3.321142 & -0.135998 \\ 1 & -6.046046 & -1.914705 & 1.259846 \\ 1 & -4.989476 & -0.582223 & 1.798567 \\ 1 & -5.607379 & -0.612235 & 0.123038 \\ 1 & -3.703458 & 0.629228 & 0.347980 \\ 1 & 6.221294 & -1.044449 & 1.309859\end{array}$

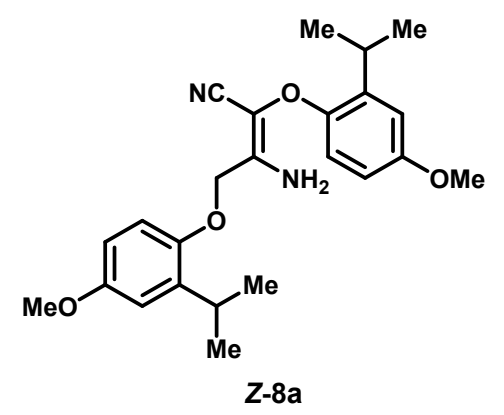

\section{Structure: $(z)-8 a$}

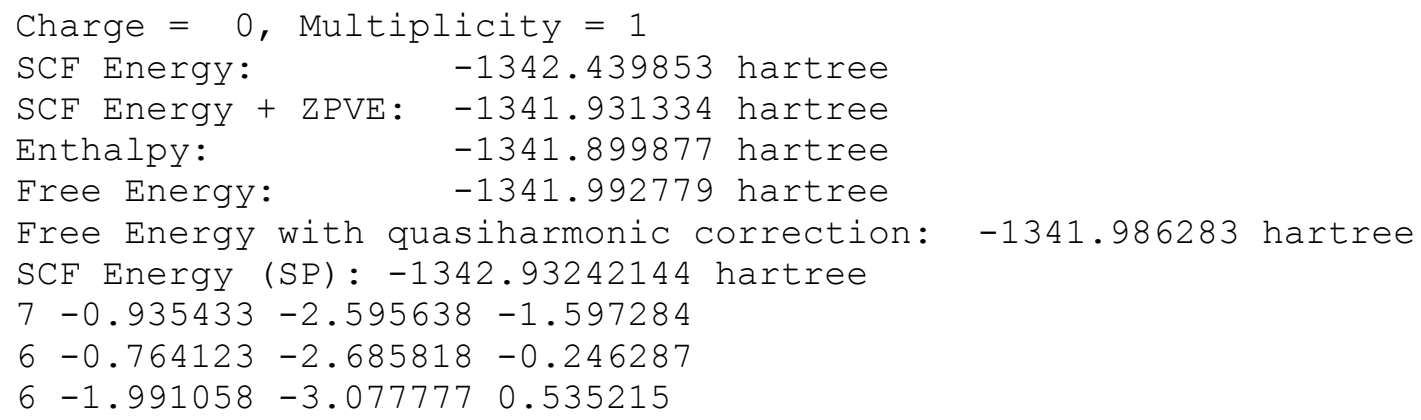




$$
\begin{array}{llll}
8 & -3.027771 & -2.125942 & 0.314789 \\
6 & -2.695944 & -0.817192 & 0.624655 \\
6 & -2.074421 & -0.494135 & 1.826330 \\
6 & -1.684420 & 0.813404 & 2.100670 \\
6 & -1.947066 & 1.811638 & 1.163332 \\
8 & -1.565732 & 3.114128 & 1.295748 \\
6 & -0.775139 & 3.434661 & 2.422123 \\
6 & -2.613937 & 1.490089 & -0.019304 \\
6 & -2.985698 & 0.181151 & -0.318179 \\
6 & -3.656982 & -0.137837 & -1.640611 \\
6 & -2.780443 & 0.293980 & -2.822728 \\
6 & -5.043599 & 0.510194 & -1.725023 \\
6 & 0.419612 & -2.401793 & 0.340700 \\
8 & 1.493430 & -2.005134 & -0.430910 \\
6 & 1.758745 & -0.631260 & -0.439305 \\
6 & 0.742481 & 0.304947 & -0.369019 \\
6 & 1.037672 & 1.668955 & -0.423163 \\
6 & 2.364183 & 2.066977 & -0.558551 \\
8 & 2.773136 & 3.364634 & -0.624404 \\
6 & 1.768435 & 4.352570 & -0.597805 \\
6 & 3.380680 & 1.106439 & -0.635143 \\
6 & 3.103750 & -0.252719 & -0.572472 \\
6 & 4.182946 & -1.319221 & -0.590651 \\
6 & 4.393662 & -1.864741 & 0.829406 \\
6 & 5.505992 & -0.832721 & -1.179036 \\
6 & 0.617108 & -2.454296 & 1.755492 \\
7 & 0.769095 & -2.476040 & 2.905711 \\
1 & -1.878189 & -2.434172 & -1.921604 \\
1 & -0.199180 & -2.141613 & -2.119551 \\
1 & -2.386675 & -4.026570 & 0.163798 \\
1 & -1.753878 & -3.190160 & 1.596448 \\
1 & -1.849909 & -1.267539 & 2.553119 \\
1 & -1.163697 & 1.023163 & 3.026172 \\
1 & -0.545822 & 4.497178 & 2.341089 \\
1 & -1.318138 & 3.255436 & 3.357261 \\
1 & 0.156587 & 2.854612 & 2.424840 \\
1 & -2.807369 & 2.293771 & -0.724278 \\
1 & -3.797319 & -1.222775 & -1.682142 \\
1 & -3.218746 & -0.034680 & -3.769780 \\
1 & -2.685618 & 1.383604 & -2.861230 \\
1 & -1.769731 & -0.117784 & -2.739559 \\
1 & -5.681151 & 0.181042 & -0.901005 \\
1 & -4.964048 & 1.601025 & -1.676502 \\
1 & -5.533205 & 0.249478 & -2.668029 \\
1 & -0.289317 & -0.015396 & -0.265851 \\
1 & 0.227225 & 2.386284 & -0.356897 \\
1 & 1.083145 & 4.249229 & -1.447878 \\
1 & 1.183937 & 4.308036 & 0.329854 \\
1 & 2.280610 & 5.312401 & -0.658074 \\
1 & 4.398752 & 1.464090 & -0.742665 \\
1 & 3.811598 & -2.142311 & -1.211895 \\
1 & 5.136880 & -2.667822 & 0.825178 \\
1 & 4.753946 & -1.067495 & 1.487457 \\
1 & 3.464088 & -2.257803 & 1.247947
\end{array}
$$


$15.370804-0.390238-2.170002$

$15.976960-0.085388-0.532049$

$16.202717-1.670333-1.268800$<smiles>COc1ccc(OC/C(N)=C(/C#N)Oc2ccc(OC)cc2C(C)C)c(C(C)C)c1</smiles>

$E-8 \mathrm{a}$

\section{Structure: $(E)-8 a$}

Charge $=0$, Multiplicity $=1$

SCF Energy: $\quad-1342.439488$ hartree

SCF Energy + ZPVE: -1341.930663 hartree

Enthalpy: $\quad-1341.899565$ hartree

Free Energy: $\quad-1341.990190$ hartree

Free Energy with quasiharmonic correction: -1341.985206 hartree SCF Energy (SP) : -1342.93097877 hartree

$\begin{array}{lllll}7 & 3.898816 & -1.827373 & 0.526465\end{array}$

$\begin{array}{lllll}6 & 2.618018 & -1.864444 & 0.030879\end{array}$

$\begin{array}{lllll}6 & 2.497848 & -1.549199 & -1.438955\end{array}$

$\begin{array}{llll}8 & 2.711151 & -0.163348 & -1.675775\end{array}$

$\begin{array}{lllll}6 & 1.606220 & 0.638395 & -1.423489\end{array}$

$\begin{array}{llllll}6 & 0.426997 & 0.444697 & -2.125973\end{array}$

$\begin{array}{llll}6 & -0.692342 & 1.236580 & -1.876127\end{array}$

$\begin{array}{lllll}6 & -0.598827 & 2.249580 & -0.926487\end{array}$

$\begin{array}{lllll}8 & -1.631993 & 3.060542 & -0.565582\end{array}$

$\begin{array}{lllll}6 & -2.858324 & 2.882791 & -1.246413\end{array}$

$\begin{array}{llllll}6 & 0.608287 & 2.469861 & -0.255034\end{array}$

$\begin{array}{lllll}6 & 1.719800 & 1.664293 & -0.471472\end{array}$

$\begin{array}{llll}6 & 3.030473 & 1.859889 & 0.267197\end{array}$

$\begin{array}{lllll}6 & 2.892715 & 2.691352 & 1.540934\end{array}$

$\begin{array}{lllll}6 & 4.081069 & 2.473870 & -0.668475\end{array}$

$\begin{array}{lllll}6 & 1.536157 & -2.201628 & 0.769522\end{array}$

$\begin{array}{lllll}6 & 1.683752 & -2.479687 & 2.168549\end{array}$

$\begin{array}{llll}7 & 1.887505 & -2.667422 & 3.294803\end{array}$

$\begin{array}{lllll}8 & 0.288532 & -2.339093 & 0.205946\end{array}$

$\begin{array}{lllll}6 & -0.679235 & -1.407213 & 0.566781\end{array}$

$\begin{array}{lllll}6 & -1.970893 & -1.645620 & 0.064427\end{array}$

$\begin{array}{lllll}6 & -2.227823 & -2.861224 & -0.806510\end{array}$

$\begin{array}{lllll}6 & -3.700744 & -3.262334 & -0.869276\end{array}$

$\begin{array}{lllll}6 & -1.679302 & -2.621181 & -2.219328\end{array}$

$\begin{array}{llll}6 & -2.958729 & -0.717976 & 0.363445\end{array}$

$\begin{array}{llll}6 & -2.695389 & 0.409693 & 1.151539\end{array}$

$\begin{array}{llll}8 & -3.749700 & 1.252826 & 1.347256\end{array}$

$\begin{array}{llll}6 & -3.504894 & 2.411820 & 2.116773\end{array}$

$\begin{array}{lllll}6 & -1.420314 & 0.608541 & 1.663148\end{array}$

$\begin{array}{llll}6 & -0.409298 & -0.304944 & 1.358853\end{array}$

$\begin{array}{lllll}1 & 4.522857 & -1.199416 & 0.038358\end{array}$ 
$14.004355-1.8183591 .532978$

$11.525184-1.875754-1.809162$

$13.286873-2.077797-1.981000$

$10.372946-0.336305-2.878165$

$\begin{array}{llll}1 & -1.612288 & 1.045498 & -2.414207\end{array}$

$\begin{array}{llll}1 & -3.252792 & 1.871767 & -1.095784\end{array}$

$\begin{array}{lllll}1 & -3.552105 & 3.604627 & -0.814950\end{array}$

$\begin{array}{lllll}1 & -2.744420 & 3.081609 & -2.318797\end{array}$

$\begin{array}{lllll}1 & 0.630319 & 3.268358 & 0.478843\end{array}$

$\begin{array}{lllll}1 & 3.380387 & 0.859046 & 0.552033\end{array}$

$\begin{array}{lllll}1 & 3.839911 & 2.698847 & 2.086726\end{array}$

$\begin{array}{llll}1 & 2.640937 & 3.731434 & 1.309400\end{array}$

$\begin{array}{lllll}1 & 2.118784 & 2.292487 & 2.203889\end{array}$

$\begin{array}{lllll}1 & 3.756123 & 3.466871 & -0.995133\end{array}$

$\begin{array}{llll}1 & 5.041020 & 2.582452 & -0.153732\end{array}$

$\begin{array}{lllll}1 & 4.226343 & 1.851597 & -1.554283\end{array}$

$1-1.663304-3.692242-0.368209$

$1-3.805069-4.211819-1.401077$

$1-4.293519-2.518363-1.411741$

$\begin{array}{lllll}1 & -4.129383 & -3.378518 & 0.129695\end{array}$

$\begin{array}{llll}1 & -2.184929 & -1.764597 & -2.679225\end{array}$

$1-1.841408-3.497787-2.853854$

$1-0.607697-2.411323-2.187460$

$\begin{array}{lllll}1 & -3.973536 & -0.849497 & 0.002959\end{array}$

$\begin{array}{lllll}1 & -2.726967 & 3.031381 & 1.654790\end{array}$

$\begin{array}{llll}1 & -3.206600 & 2.152714 & 3.140031\end{array}$

$1-4.446135 \quad 2.960960 \quad 2.146435$

$\begin{array}{llll}1 & -1.179799 & 1.476963 & 2.263974\end{array}$

$\begin{array}{lllll}1 & 0.594400 & -0.124167 & 1.725986\end{array}$ 


\subsection{DFT Calculations and Analysis of NOESY Spectrum for Assignment of the Stereochemistry of Compound 8a}

Calculation details related to predicted ${ }^{13} \mathrm{C}$ NMR chemical shifts and relative energies in chloroform

Conformer searches were performed using Macromodel (Schrodinger 2019-4) ${ }^{6}$ mixed mode searches with the OPLS3e force field for both stereoisomers. The lowest energy conformers within $10 \mathrm{kcal} / \mathrm{mol}$ were then subjected to DFT calculations using Gaussian ' $16 .{ }^{2}$ Geometries, energies and vibrational frequencies were calculated at the B3LYP-D3/6-311G(d,p) level with implicit solvent (polarizable continuum model, PCM) for chloroform. A vibrational scaling factor of 0.986 was used for frequencies, consistent with this model chemistry. ${ }^{7}$ Carbon chemical shifts were then calculated at the $\omega$ B97X-D/def2-SVP level of theory using linear scaling factors, $\delta=(b-\sigma) /(-m)$, (y-intercept, $b=196.0386$, slope, $m=-1.0065)$ to convert the magnetic shielding values $(\sigma)$ to chemical shifts $(\delta, \mathrm{ppm})$. The scaling factors were obtained from linear regression of experimental to calculated NMR data for a large test set of model compounds. ${ }^{8}$ The calculated chemical shifts were then weighted according the calculated Boltzmann energy distribution.

A. Relative Energies of $\mathbf{E}-\mathbf{8 a}$ and Z-8a based on DFT calculations in $\mathrm{CDCl}_{3}$<smiles>COc1ccc(OC/C(N)=C(/C#N)Oc2ccc(OC)cc2C(C)C)c(C(C)C)c1</smiles>

$G=1.4 \mathrm{kcal} / \mathrm{mol}$

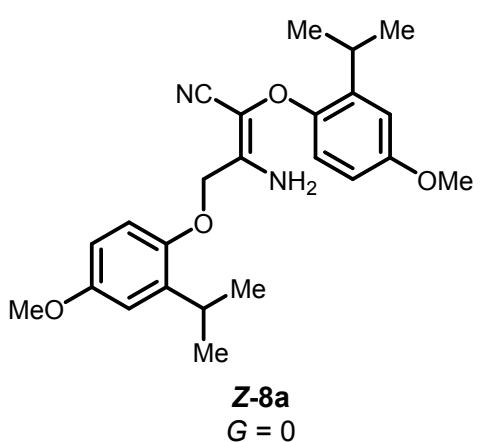

B. Atom numbering scheme related to DFT predicted ${ }^{13} \mathrm{C}$ NMR chemical shift<smiles>COc1ccc(OC/C(N)=C(/C#N)Oc2ccc(OC)cc2C(C)C)c(C(C)C)c1</smiles>

$E-8 a$<smiles>COc1ccc(OC/C(N)=C(/C#N)Oc2ccc(OC)cc2C(C)C)c(C(C)C)c1</smiles>

Figure S53. (A) DFT predicted relative energy $\boldsymbol{E}-8$ a relative to $\boldsymbol{Z}-\mathbf{8 a}$ calculated using B3LYP-D3/6$311 \mathrm{G}(\mathrm{d}, \mathrm{p})$ level with implicit solvent (polarizable continuum model) for chloroform. (B) Atom numbering scheme for $\boldsymbol{E - 8 a}$ and $\boldsymbol{Z - 8 a}$ related to DFT predicted NMR chemical shifts. 
Table S1. Comparison of calculated ${ }^{a}$ vs. experimental carbon chemical shifts. ${ }^{b}$

\begin{tabular}{|c|c|c|c|c|c|}
\hline \multirow{2}{*}{ Atom } & \multirow{2}{*}{$\operatorname{Expt} \delta_{\mathrm{C}}(\mathrm{ppm})$} & \multicolumn{2}{|c|}{ DFT $\delta_{\mathrm{C}}(\mathrm{ppm})$} & \multicolumn{2}{|c|}{ Error (ppm) } \\
\hline & & $E-8 \mathbf{a}$ & $Z-8 \mathbf{a}$ & $E-8 a$ & $Z-8 \mathbf{a}$ \\
\hline 1 & 147.0 & 148.2 & 145.3 & 1.2 & -1.7 \\
\hline 2 & 139.3 & 138.9 & 138.3 & -0.4 & -1.0 \\
\hline 3 & 113.4 & 115.3 & 114.7 & 1.9 & 1.3 \\
\hline 4 & 156.0 & 154.4 & 154.2 & -1.5 & -1.7 \\
\hline 5 & 110.9 & 107.5 & 107.1 & -3.3 & -3.8 \\
\hline 6 & 115.3 & 113.3 & 113.3 & -2.0 & -2.0 \\
\hline 8 & 103.8 & 97.5 & 103.0 & -6.3 & -0.9 \\
\hline 9 & 145.4 & 156.4 & 150.9 & 10.9 & 5.5 \\
\hline 11 & 27.3 & 28.8 & 30.1 & 1.5 & 2.7 \\
\hline 12,25 & 23.0 & 23.1 & 22.8 & 0.1 & -0.2 \\
\hline 14 & 55.8 & 53.9 & 53.9 & -1.8 & -1.8 \\
\hline 15 & 116.0 & 112.6 & 113.7 & -3.5 & -2.4 \\
\hline 17 & 65.5 & 67.8 & 66.9 & 2.3 & 1.3 \\
\hline 19 & 148.8 & 146.4 & 144.9 & -2.4 & -3.9 \\
\hline 20 & 139.1 & 143.3 & 143.7 & 4.2 & 4.6 \\
\hline 21 & 113.6 & 109.7 & 114.9 & -3.8 & 1.4 \\
\hline 22 & 155.1 & 155.8 & 154.9 & 0.7 & -0.2 \\
\hline 23 & 110.5 & 113.0 & 111.1 & 2.6 & 0.6 \\
\hline 24 & 113.6 & 121.4 & 121.4 & 7.8 & 7.8 \\
\hline 26 & 27.2 & 28.8 & 28.0 & 1.6 & 0.8 \\
\hline 27,30 & 23.0 & 23.6 & 24.9 & 0.6 & 1.9 \\
\hline \multirow[t]{2}{*}{29} & 55.8 & 53.7 & 54.2 & -2.1 & -1.6 \\
\hline & & & RMSD: & 3.8 & 2.9 \\
\hline
\end{tabular}

a:DFT model chemistry: PCM- $\omega$ B97X-D/def2-SVP//B3LYP-D3/6-311G(d,p). See Figure S53B for atom numbering scheme. ${ }^{b} \mathrm{RMSD}=$ root mean square deviation. 


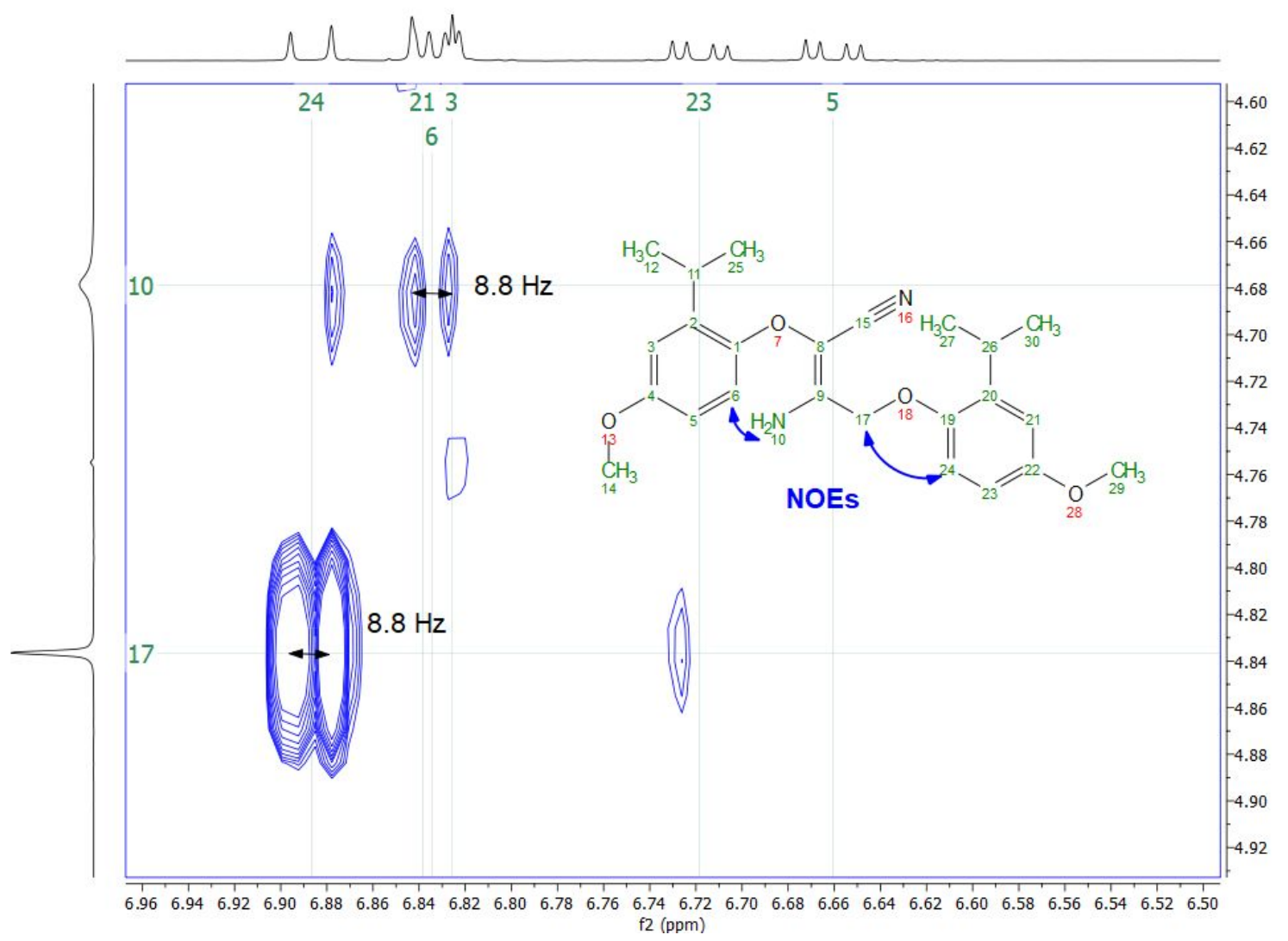

Figure S54. ${ }^{1} \mathrm{H} /{ }^{1} \mathrm{H}$ NOESY $\left(600 / 600 \mathrm{MHz}, 500 \mathrm{~ms}\right.$ mixing time $\mathrm{CDCl}_{3}$ ) spectrum for 8a (zoomed into region showing key NOEs)

The NOESY spectrum above shows a strong NOE between protons 24 and 17 and a medium intensity NOE between protons 10 and 6 . This second NOE is consistent with the Z-isomer configuration.

\section{Conclusions}

The root mean standard deviation (RMSD) error for the $Z$-isomer of $\mathbf{8 a}$ is significantly lower than that of the $E$-isomer ( 2.9 versus 3.8 , see Table $\mathrm{S} 1$ ), which shows that the $Z$-isomer is most consistent with the experimental data. In addition, the $Z$-isomer of 8 a was found to be $4.4 \mathrm{kcal} / \mathrm{mol}$ lower in energy than the $E$ isomer. Finally, a medium intensity NOE was observed between the $-\mathrm{NH}_{2}$ protons and aromatic protons, position 6. Thus, the NOE and DFT data are both consistent with the $\boldsymbol{Z - 8 a}$ structure. 
<smiles>COc1ccc(OC/C(N)=C(/C#N)Oc2ccc(OC)cc2C(C)C)c(C(C)C)c1</smiles>

$E-8 a$

Structure: $\boldsymbol{E - 8 a}$ (in chloroform, see below for model chemistry)

Model chemistry: B3LYP-D3/6-311G(d,p) PCM=chloroform

Charge $=0$, Multiplicity $=1$

SCF Energy: $\quad-1343.387703$ hartree

SCF Energy + ZPVE: -1342.894490 hartree

Enthalpy: $\quad-1342.862085$ hartree

Free Energy: $\quad-1342.957609$ hartree

Free Energy with quasiharmonic correction: -1342.950607 hartree
$\begin{array}{lllll}6 & 3.102860 & -1.879787 & 0.517397\end{array}$
$\begin{array}{lll}6 & 2.843728-2.203476-0.821582\end{array}$
$\begin{array}{lll}6 & 2.358783-1.224523-1.684269\end{array}$
$\begin{array}{llll}6 & 2.126059 & 0.063998 & -1.194094\end{array}$
$\begin{array}{llll}6 & 2.374225 & 0.364239 & 0.135328\end{array}$
$\begin{array}{lllll}6 & 2.876881 & -0.605963 & 1.023231\end{array}$
$\begin{array}{llll}6 & 3.106260 & -0.247226 & 2.483402\end{array}$
$\begin{array}{llll}8 & 2.114401 & 1.618737 & 0.693215\end{array}$
$\begin{array}{lllll}6 & 1.781520 & 2.660177 & -0.164985\end{array}$
$\begin{array}{llllll}6 & 0.480781 & 2.999274 & -0.385186\end{array}$
$\begin{array}{lllll}7 & 0.072814 & 4.073682 & -1.115018\end{array}$
$\begin{array}{llll}6 & -0.630476 & 2.202456 & 0.245865\end{array}$
$8-1.5849451 .920290-0.788565$
$6-2.6739391 .160909-0.366725$
$\begin{array}{llll}6 & -3.844280 & 1.829521 & -0.003880\end{array}$
$\begin{array}{lllll}6 & -4.967835 & 1.118306 & 0.383382\end{array}$
$6-4.921992-0.2816090 .415606$
$6-3.748801-0.943816 \quad 0.051810$
$6-2.605507-0.234950-0.346420$
$6-1.336477-0.975640-0.735454$
$\begin{array}{llll}8 & 3.091888 & -3.501683 & -1.170831\end{array}$
$\begin{array}{llll}6 & 2.824863 & -3.897525 & -2.513218\end{array}$
$\begin{array}{llll}6 & 1.763585 & -0.220358 & 3.241228\end{array}$

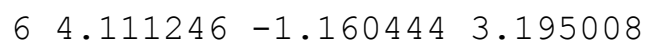
$\begin{array}{lllll}6 & 2.857243 & 3.368269 & -0.754750\end{array}$
$\begin{array}{lllll}7 & 3.718336 & 3.961321 & -1.255793\end{array}$
$\begin{array}{llll}8 & -6.070998 & -0.901642 & 0.811769\end{array}$
$\begin{array}{llll}6 & -6.091639 & -2.326318 & 0.864133\end{array}$
$\begin{array}{lllll}6 & -0.729903 & -1.721484 & 0.465691\end{array}$
$\begin{array}{lllll}6 & -1.564467 & -1.932132 & -1.917859\end{array}$
$\begin{array}{lllll}1 & 3.485141 & -2.667578 & 1.153094\end{array}$
$\begin{array}{llll}1 & 2.150197 & -1.435062 & -2.723530\end{array}$
$\begin{array}{lllll}1 & 1.740656 & 0.825103 & -1.859267\end{array}$
$\begin{array}{lllll}1 & 3.509015 & 0.770357 & 2.497631\end{array}$
$10.742909 \quad 4.545903-1.705027$ 


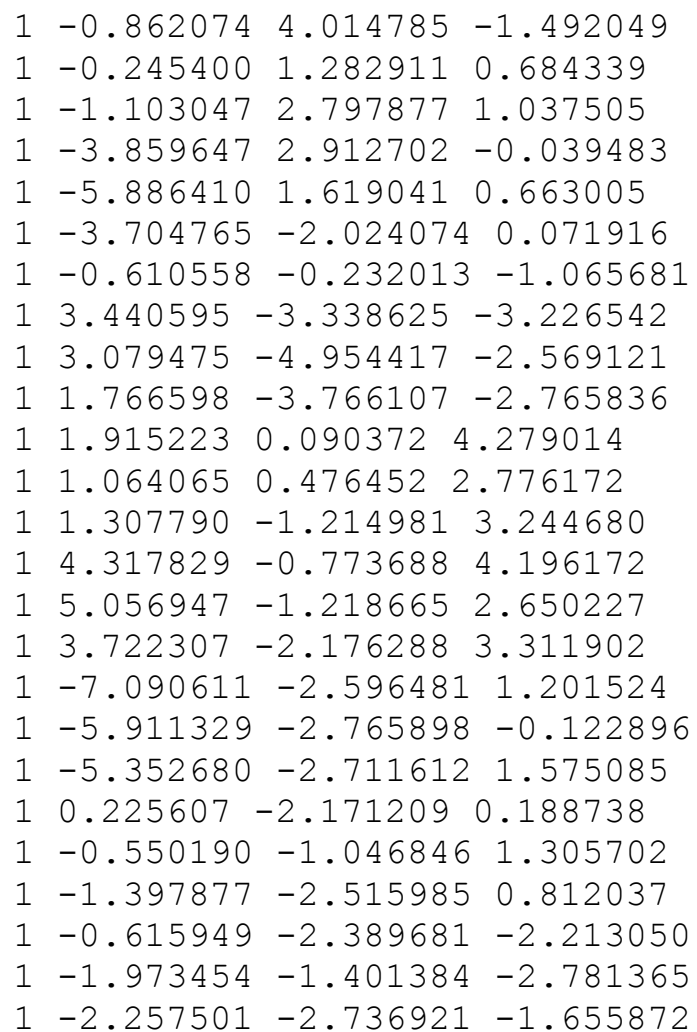<smiles>COc1ccc(OC/C(N)=C(\C#N)Oc2ccc(OC)cc2C(C)C)c(C(C)C)c1</smiles>

Structure: $\boldsymbol{Z - 8 a}$ (in chloroform, see below for model chemistry) Model chemistry: B3LYP-D3/6-311G(d,P) PCM=chloroform Charge $=0$, Multiplicity $=1$

SCF Energy: $\quad-1343.394159$ hartree

SCF Energy + ZPVE: -1342.900795 hartree

Enthalpy: $\quad-1342.868392$ hartree

Free Energy: $\quad-1342.964556$ hartree

Free Energy with quasiharmonic correction: -1342.956605 hartree
$\begin{array}{llll}6 & 0.401142-2.325870 & 0.674719\end{array}$
$81.560476-1.967740-0.004573$
$\begin{array}{lllll}6 & 4.215767-1.305297-0.529094\end{array}$
$63.139720-0.232978-0.447739$
$61.815091-0.593930-0.145296$
$\begin{array}{llll}6 & 0.816467 & 0.356793 & -0.019150\end{array}$
$\begin{array}{lllll}6 & 1.103984 & 1.713561 & -0.184518\end{array}$
$\begin{array}{lllll}6 & 2.408788 & 2.096983 & -0.486056\end{array}$
$\begin{array}{llll}7 & -0.648945 & -2.854697 & -1.385099\end{array}$ 


$\begin{array}{llll}6 & -1.989618 & -3.100525 & 0.631196 \\ 8 & -3.023608 & -2.190661 & 0.217325 \\ 6 & -2.788167 & -0.843354 & 0.477897 \\ 6 & -2.461718 & -0.406850 & 1.758302 \\ 6 & -2.180068 & 0.934149 & 2.010617 \\ 6 & -2.247571 & 1.850789 & 0.959695 \\ 6 & -2.628360 & 1.414935 & -0.310520 \\ 6 & -2.891700 & 0.073392 & -0.582166 \\ 6 & -3.262861 & -0.363884 & -1.989819 \\ 6 & 3.407083 & 1.121524 & -0.618639 \\ 6 & -0.679384 & -2.753047 & -0.032720 \\ 6 & 0.430252 & -2.214823 & 2.085074 \\ 7 & 0.481875 & -2.102950 & 3.237834 \\ 6 & 4.613737 & -1.758666 & 0.890153 \\ 6 & 5.452041 & -0.885416 & -1.332560 \\ 8 & 2.816732 & 3.388896 & -0.670906 \\ 6 & 1.844300 & 4.421368 & -0.530912 \\ 8 & -1.928182 & 3.177157 & 1.058714 \\ 6 & -1.467410 & 3.665649 & 2.318182 \\ 6 & -4.616593 & 0.229087 & -2.418675 \\ 6 & -2.157705 & -0.011114 & -3.000943 \\ 1 & 3.767637 & -2.168117 & -1.032346 \\ 1 & -0.194332 & 0.054398 & 0.209519 \\ 1 & 0.305303 & 2.433189 & -0.071266 \\ 1 & 0.142247 & -2.479870 & -1.885154 \\ 1 & -1.519702 & -2.916095 & -1.886563 \\ 1 & -2.331551 & -4.083323 & 0.303689 \\ 1 & -1.880717 & -3.118914 & 1.716584 \\ 1 & -2.391851 & -1.118563 & 2.570664 \\ 1 & -1.892610 & 1.233499 & 3.008300 \\ 1 & -2.678382 & 2.153649 & -1.101429 \\ 1 & -3.375223 & -1.449396 & -1.971119 \\ 1 & 4.406765 & 1.462227 & -0.854452 \\ 1 & 3.743588 & -2.106987 & 1.449159 \\ 1 & 5.341073 & -2.574512 & 0.842544 \\ 1 & 5.066541 & -0.928659 & 1.440954 \\ 1 & 5.182552 & -0.530418 & -2.330756 \\ 1 & 6.127770 & -1.737190 & -1.444603 \\ 1 & 6.010081 & -0.090842 & -0.828557 \\ 1 & 2.372345 & 5.355748 & -0.713035 \\ 1 & 1.035227 & 4.312887 & -1.261634 \\ 1 & 1.418279 & 4.435595 & 0.478495 \\ 1 & -1.255171 & 4.722458 & 2.166742 \\ 1 & -2.232860 & 3.557405 & 3.093478 \\ 1 & -0.552663 & 3.151423 & 2.631529 \\ 1 & -4.894481 & -0.132522 & -3.412906 \\ 1 & -5.407303 & -0.050869 & -1.718210 \\ 1 & -4.571752 & 1.321321 & -2.458119 \\ 1 & -2.401737 & -0.412663 & -3.988640 \\ 1 & -1.193938 & -0.419059 & -2.688660 \\ 1 & -2.042291 & 1.071642 & -3.100008\end{array}$




\section{References}

(1) Gaussian 09, Revision D.01, M. J. Frisch, G. W. Trucks, H. B. Schlegel, G. E. Scuseria, M. A. Robb, J. R. Cheeseman, G. Scalmani, V. Barone, G. A. Petersson, H. Nakatsuji, X. Li, M. Caricato, A. Marenich, J. Bloino, B. G. Janesko, R. Gomperts, B. Mennucci, H. P. Hratchian, J. V. Ortiz, A. F. Izmaylov, J. L. Sonnenberg, D. Williams-Young, F. Ding, F. Lipparini, F. Egidi, J. Goings, B. Peng, A. Petrone, T. Henderson, D. Ranasinghe, V. G. Zakrzewski, J. Gao, N. Rega, G. Zheng, W. Liang, M. Hada, M. Ehara, K. Toyota, R. Fukuda, J. Hasegawa, M. Ishida, T. Nakajima, Y. Honda, O. Kitao, H. Nakai, T. Vreven, K. Throssell, J. A. Montgomery, Jr., J. E. Peralta, F. Ogliaro, M. Bearpark, J. J. Heyd, E. Brothers, K. N. Kudin, V. N. Staroverov, T. Keith, R. Kobayashi, J. Normand, K. Raghavachari, A. Rendell, J. C. Burant, S. S. Iyengar, J. Tomasi, M. Cossi, J. M. Millam, M. Klene, C. Adamo, R. Cammi, J. W. Ochterski, R. L. Martin, K. Morokuma, O. Farkas, J. B. Foresman, and D. J. Fox, Gaussian, Inc., Wallingford CT, 2016.

(2) Gaussian 16, Revision A.03, Frisch, M. J.; Trucks, G. W.; Schlegel, H. B.; Scuseria, G. E.; Robb, M. A.; Cheeseman, J. R.; Scalmani, G.; Barone, V.; Petersson, G. A.; Nakatsuji, H.; Li, X.; Caricato, M.; Marenich, A. V.; Bloino, J.; Janesko, B. G.; Gomperts, R.; Mennucci, B.; Hratchian, H. P.; Ortiz, J. V.; Izmaylov, A. F.; Sonnenberg, J. L.; Williams-Young, D.; Ding, F.; Lipparini, F.; Egidi, F.; Goings, J.; Peng, B.; Petrone, A.; Henderson, T.; Ranasinghe, D.; Zakrzewski, V. G.; Gao, J.; Rega, N.; Zheng, G.; Liang, W.; Hada, M.; Ehara, M.; Toyota, K.; Fukuda, R.; Hasegawa, J.; Ishida, M.; Nakajima, T.; Honda, Y.; Kitao, O.; Nakai, H.; Vreven, T.; Throssell, K.; Montgomery, J. A., Jr.; Peralta, J. E.; Ogliaro, F.; Bearpark, M. J.; Heyd, J. J.; Brothers, E. N.; Kudin, K. N.; Staroverov, V. N.; Keith, T. A.; Kobayashi, R.; Normand, J.; Raghavachari, K.; Rendell, A. P.; Burant, J. C.; Iyengar, S. S.; Tomasi, J.; Cossi, M.; Millam, J. M.; Klene, M.; Adamo, C.; Cammi, R.; Ochterski, J. W.; Martin, R. L.; Morokuma, K.; Farkas, O.; Foresman, J. B.; Fox, D. J. Gaussian, Inc., Wallingford CT, 2016.

(3) Zhao, Y.; Truhlar, D. G. The M06 Suite of Density Functionals for Main Group Thermochemistry, Thermochemical Kinetics, Noncovalent Interactions, Excited States, and Transition Elements: Two New Functionals and Systematic Testing of Four M06-Class Functionals and 12 Other Functionals. Theor. Chem. Acc. 2008, 120, 215-241.

(4) Weigend, F.; Ahlrichs, R. Balanced Basis Sets of Split Valence, Triple Zeta Valence and Quadruple Zeta Valence Quality for H to Rn: Design and Assessment of Accuracy. Phys. Chem. Chem. Phys. 2005, 7, 3297-3305.

(5) Ribeiro, R. F.; Marenich, A. V.; Cramer, C. J.; Truhlar, D. G. Use of Solution-Phase Vibrational Frequencies in Continuum Models for the Free Energy of Solvation. J. Phys. Chem. B 2011, 115, 14556-14562.

(6) Schrödinger Release 2019-4, MacroModel, Schrödinger, LLC, New York, 2019.

(7) National Institute of Standards and Technology, Computational Chemistry Comparison and Benchmark Database, Release 20, August 2019, http://cccbdb.nist.gov/vibscalejust.asp

(8) Lodewyk, M. W.; Siebert, M. R.; Tantillo, D. J. Computational Prediction of ${ }^{1} \mathrm{H}$ and ${ }^{13} \mathrm{C}$ Chemical Shifts: A Useful Tool for Natural Product, Mechanistic, and Synthetic Organic Chemistry. Chem. Rev. 2012, 112, 1839-1862. 